\title{
NLO QCD+EW predictions for $V+$ jets including off-shell vector-boson decays and multijet merging
}

\author{
S. Kallweit, ${ }^{a}$ J.M. Lindert, ${ }^{b}$ P. Maierhöfer, ${ }^{c, d}$ S. Pozzorini ${ }^{b}$ and M. Schönherr ${ }^{b}$ \\ ${ }^{a}$ Institut für Physik \& PRISMA Cluster of Excellence, \\ Johannes Gutenberg Universität, 55099 Mainz, Germany \\ ${ }^{b}$ Physik-Institut, Universität Zürich, \\ Winterthurerstrasse 190, CH-8057 Zürich, Switzerland \\ ${ }^{c}$ Institute for Particle Physics Phenomenology, Durham University, \\ Durham DH1 3LE, U.K. \\ ${ }^{d}$ Physikalisches Institut, Albert-Ludwigs-Universität Freiburg, \\ 79104 Freiburg, Germany \\ E-mail: kallweit@uni-mainz.de, lindert@physik.uzh.ch, \\ philipp.maierhoefer@physik.uni-freiburg.de, pozzorin@physik.uzh.ch, \\ marek.schoenherr@physik.uzh.ch
}

ABSTRACT: We present next-to-leading order (NLO) predictions including QCD and electroweak (EW) corrections for the production and decay of off-shell electroweak vector bosons in association with up to two jets at the $13 \mathrm{TeV}$ LHC. All possible dilepton final states with zero, one or two charged leptons that can arise from off-shell $\mathrm{W}$ and $\mathrm{Z}$ bosons or photons are considered. All predictions are obtained using the automated implementation of NLO QCD+EW corrections in the OpENLOOPS matrix-element generator combined with the Munich and Sherpa Monte Carlo frameworks. Electroweak corrections play an especially important role in the context of BSM searches, due to the presence of large EW Sudakov logarithms at the $\mathrm{TeV}$ scale. In this kinematic regime, important observables such as the jet transverse momentum or the total transverse energy are strongly sensitive to multijet emissions. As a result, fixed-order NLO QCD+EW predictions are plagued by huge QCD corrections and poor theoretical precision. To remedy this problem we present an approximate method that allows for a simple and reliable implementation of NLO EW corrections in the MEPs@NLO multijet merging framework. Using this general approach we present an inclusive simulation of vector-boson production in association with jets that guarantees NLO QCD + EW accuracy in all phase-space regions involving up to two resolved jets.

KeYwORDS: NLO Computations, QCD Phenomenology

ArXIV EPRINT: 1511.08692 


\section{Contents}

1 Introduction 1

2 Technical ingredients and setup of the simulations 3

2.1 Considered processes and perturbative contributions 3

2.2 Methods and tools 5

$\begin{array}{lll}2.3 & \text { Physics objects and selection cuts } & 6\end{array}$

$\begin{array}{lll}2.4 & \text { Input parameters, scale choices and variations } & 7\end{array}$

3 Giant $K$-factors and electroweak corrections for $V+1$ jet production $\quad 9$

4 Fixed-order predictions for $V+2$ jet production $\quad 10$

$\begin{array}{lll}4.1 & \text { NLO QCD+EW predictions } & 10\end{array}$

$\begin{array}{lll}4.2 & \text { Subleading Born and photon-induced contributions } & 15\end{array}$

5 Multijet merged predictions for $V+$ jets at NLO QCD+EW 17

$\begin{array}{lll}5.1 & \text { Combining } p p \rightarrow V+1,2 \text { jets with exclusive sums } & 18\end{array}$

5.2 Virtual approximation of NLO EW corrections 22

$\begin{array}{lll}5.3 & \text { MEPS merging at NLO QCD } & 28\end{array}$

5.4 Extension of MEPs merging to NLO QCD+EW 31

5.5 Numerical MePs@NLO QCD+EW results for $p p \rightarrow V+0,1,2$ jets 32

6 Summary and conclusions $\quad 36$

A Further NLO predictions for $V+2$ jet production 37

B Multijet merging systematic uncertainties in the TeV range 38

\section{Introduction}

The production of electroweak (EW) vector bosons in association with jets plays a key role in the physics programme of the Large Hadron Collider (LHC). Inclusive and differential measurements of vector-boson plus multijet cross sections [1-6] can be performed for a wide range of jet multiplicities exploiting various clean final states that arise from the leptonic decays of $\mathrm{W}$ and $\mathrm{Z}$ bosons or off-shell photons. This offers unique opportunities to test the Standard Model at high precision and to validate fundamental aspects of theoretical simulations at hadron colliders. Associated $V+$ multijet production $(V=\mathrm{W}, \mathrm{Z})$ represents also an important background to a large variety of analyses based on signatures with leptons, missing energy and jets. In particular, it is a prominent background in searches for physics beyond the Standard Model (BSM) at the TeV scale. In this context, the 
availability of precise theoretical predictions for $V+$ multijet production can play a critical role for the sensitivity to new phenomena and for the interpretation of possible discoveries.

Predictions for $V+$ multijet production at next-to-leading order (NLO) in QCD [719] are widely available, and the precision of higher-order QCD calculations has already reached the next-to-next-to-leading order (NNLO) for $p p \rightarrow V+1$ jet $[20,21]$. Also EW corrections can play an important role. Their inclusion is mandatory for any precision measurement. Moreover, EW corrections are especially relevant at the $\mathrm{TeV}$ scale, where large logarithms of Sudakov type [22-28] can lead to NLO EW effects of tens of percent. While NLO predictions for electroweak-boson production in association with a single jet [29-37] have been available for a while, thanks to the recent progress in NLO automation also $V+$ multijet calculations at NLO EW became feasible. In particular, various algorithms for the automated generation of one-loop scattering amplitudes have proven to possess the degree of flexibility that is required in order to address NLO EW calculations [38-43]. Predictions for vector-boson plus multijet production at NLO EW are motivated by the large impact of EW Sudakov effects on BSM signatures with multiple jets [44] and, more generally, by the abundance of multijet emissions in $p p \rightarrow V+$ jets at high energy. First NLO EW predictions for vector-boson production in association with more than one jet have been presented for $p p \rightarrow \ell^{+} \ell^{-} j j$ [45] and for on-shell $\mathrm{W}^{+}$-boson production with up to three associated jets at NLO QCD+EW [41]. Independent NLO EW results for $p p \rightarrow \mathrm{W}+2$ jets have been reported in [43].

In this paper we present new NLO QCD+EW results for $p p \rightarrow V+$ jets that involve up to two jets and cover all possible signatures resulting from off-shell vector-boson decays into charged leptons or neutrinos, i.e. we perform full $2 \rightarrow 3$ and $2 \rightarrow 4$ calculations for $p p \rightarrow \ell^{+} \nu_{\ell}+1,2$ jets, $p p \rightarrow \ell^{-} \bar{\nu}_{\ell}+1,2$ jets, $p p \rightarrow \ell^{+} \ell^{-}+1,2$ jets and $p p \rightarrow \nu_{\ell} \bar{\nu}_{\ell}+1,2$ jets. For convenience, the above mentioned processes will often be denoted as $V+$ jet(s) production, while all results in this paper correspond to off-shell $\ell \ell / \ell \nu / \nu \nu+$ jet(s) production.

Our predictions are obtained within the fully automated NLO QCD+EW framework [41] provided by the OpenLoops [40, 46] generator in combination with the MU$\mathrm{NICH}^{1}$ and SHERPA [47-49] Monte Carlo programs. Off-shell effects in vector-boson decays are fully taken into account thanks to a general implementation of the complex-mass scheme [50] at NLO QCD+EW in OpenLoops. This is applicable to any process that involves the production and decay of intermediate electroweak vector bosons, top quarks and Higgs bosons.

Higher-order calculations for $p p \rightarrow V+n$ jets are obviously relevant for physical observables that involve at least $n$ hard jets, but they can play a very important role also for more inclusive observables where less than $n$ hard jets are explicitly required. Prominent examples are provided by the inclusive distributions in the transverse momentum $\left(p_{\mathrm{T}}\right)$ of the leading jet and in the total transverse energy. As is well known, the tails of such distributions receive huge contributions from multijet emissions that tend to saturate the recoil induced by the leading jet, while the vector boson remains relatively soft. As a result, NLO QCD predictions for $p p \rightarrow V+1$ jet at high jet $p_{\mathrm{T}}$ are plagued by giant $K-$ factors [51], and their accuracy is effectively reduced to leading order due to the dominance of $n$-jet final states with $n \geq 2$. In this situation it is clear that also NLO EW parton-

\footnotetext{
${ }^{1}$ Munich : a MUlti-chaNel Integrator at Swiss $(\mathrm{CH})$ precision, S. Kallweit, in preparation.
} 
level results for $p p \rightarrow V+1$ jet are not applicable as they entirely miss the dominant source of EW higher-order effects, namely Sudakov-type EW corrections to $V+$ multijet production. At fixed order in perturbation theory, the natural remedy would be given by $p p \rightarrow V+1$ jet calculations with NNLO QCD and mixed NNLO QCD-EW corrections. Very recently, mixed QCD-EW corrections of $\mathcal{O}\left(\alpha_{\mathrm{S}} \alpha\right)$ to Drell-Yan processes in the resonance region have been presented in $[52,53]$. However, a corresponding calculation for $p p \rightarrow V+1$ jet is clearly out of reach. Thus, as a viable alternative, in this paper we will adopt the multijet merging approach at NLO [54-57], which allows one to combine NLO simulations of $p p \rightarrow V+0,1, \ldots, n$ jets matched to parton showers in a way that guarantees parton-shower resummation and NLO accuracy in all phase-space regions with up to $n$ resolved jets. While multijet merging methods at NLO QCD - and applications thereof to $V+$ multijet production $[54,58]$ - are already well established, in this paper we address the inclusion of NLO EW corrections for the first time. To this end we exploit an approximate treatment of NLO EW corrections, based on exact virtual EW contributions in combination with an appropriate cancellation of infrared singularities. This allows us to implement NLO EW effects in the MEPS@NLO multijet merging framework [54] in a relatively straightforward way. The proposed approach is completely general, and we implemented it in SHERPA+OpenLoops in a fully automated way. It is ideally suited for processes and observables that receive large EW Sudakov corrections and involve sizable contributions from multijet emissions.

The paper is organised as follows. In section 2 we provide technical aspects related to the employed tools and the setup of the calculation. Giant $K$-factors for $V+1$ jet production and related issues are recapitulated in section 3. In section 4 we present fixedorder NLO QCD $+\mathrm{EW}$ predictions for $p p \rightarrow V+2$ jets including all channels with off-shell $\mathrm{W}$ or $\mathrm{Z} / \gamma^{*}$ decays to leptons and neutrinos. The merging of NLO QCD+EW predictions for processes with variable jet multiplicity is addressed in section 5 , which starts with an illustration of NLO merging features based on the exclusive-sums approach at parton level. In the following we introduce and validate an approximation of NLO EW corrections which is then used in order to inject NLO EW precision into the MEPs@NLO framework. First MEPS@NLO predictions with NLO QCD+EW accuracy are presented for $p p \rightarrow \ell^{-} \bar{\nu}_{\ell}+$ jets including NLO matrix elements with up to two final-state jets. Our conclusions can be found in section 6 .

\section{Technical ingredients and setup of the simulations}

This section deals with technical aspects of the simulations. The reader might decide to skip it and to proceed directly to the presentation of physics results in sections 3-6.

\subsection{Considered processes and perturbative contributions}

In this paper we study the production and decay of electroweak bosons $\left(V=\mathrm{W}^{ \pm}, \mathrm{Z} / \gamma^{*}\right)$ in association with one and two jets at NLO QCD+EW, including off-shell effects and taking into account all decay channels with leptons and neutrinos, i.e. we address off-shell $2 \rightarrow 3$ and $2 \rightarrow 4$ processes with $\mathrm{W}^{+} \rightarrow \ell^{+} \nu_{\ell}, \mathrm{W}^{-} \rightarrow \ell^{-} \bar{\nu}_{\ell}, \mathrm{Z} / \gamma^{*} \rightarrow \ell^{+} \ell^{-}$and $\mathrm{Z} \rightarrow \nu_{\ell} \bar{\nu}_{\ell}$ final states in combination with jets. In the case of charged leptons, only one generation 


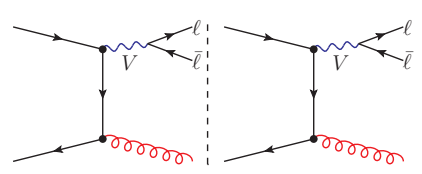

(a) $\mathcal{O}\left(\alpha_{\mathrm{S}} \alpha^{2}\right) \mathrm{LO}$

Figure 1. Representative LO contribution to $V+1$ jet production.

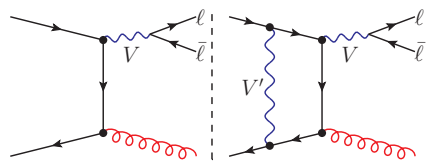

(a) Virtual $\mathcal{O}\left(\alpha_{\mathrm{S}} \alpha^{3}\right)$ correction

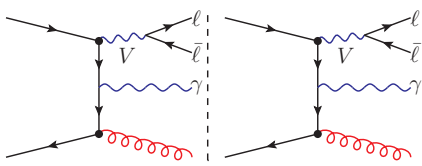

(b) Real $\mathcal{O}\left(\alpha_{\mathrm{S}} \alpha^{3}\right)$ correction

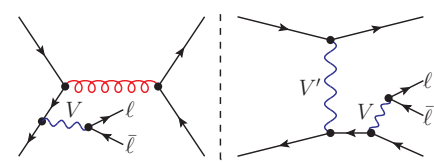

(c) Real $\mathcal{O}\left(\alpha_{\mathrm{S}} \alpha^{3}\right)$ correction

Figure 2. Representative virtual and real NLO EW contributions to $V+1$ jet production.

is included, whereas for invisible Z-boson decays all neutrino species $\left(\nu_{e}, \nu_{\mu}, \nu_{\tau}\right)$ are taken into account trivially.

In general, NLO QCD and EW corrections have to be understood within a mixed coupling expansion in $\alpha$ and $\alpha_{\mathrm{S}}$, where Born and one-loop scattering amplitudes for a given process consist of towers of $\mathcal{O}\left(\alpha_{\mathrm{S}}^{N} \alpha^{M}\right)$ contributions with a fixed overall order $N+M$ that is distributed among QCD and EW couplings in different possible $(N, M)$ combinations.

The production and off-shell decay of $V+1$ jet involves a unique LO contribution of $\mathcal{O}\left(\alpha_{\mathrm{S}} \alpha^{2}\right)$ and receives NLO QCD corrections of $\mathcal{O}\left(\alpha_{\mathrm{S}}^{2} \alpha^{2}\right)$ and NLO EW corrections of $\mathcal{O}\left(\alpha_{\mathrm{S}} \alpha^{3}\right)$. Representative Feynman diagrams are illustrated in figures 1 and 2. Here it is important to keep in mind a somewhat counter-intuitive feature of NLO EW corrections, namely that real emission at $\mathcal{O}\left(\alpha_{\mathrm{S}} \alpha^{3}\right)$ does not only involve photon bremsstrahlung (figure 2(b)) but also $V+2$ jet final states resulting from the emission of quarks through mixed QCD-EW interference terms (figure 2(c)).

The LO production and off-shell decay of $V+2$ jets receives contributions from a tower of $\mathcal{O}\left(\alpha_{\mathrm{S}}^{k} \alpha^{4-k}\right)$ terms with powers $k=2,1,0$ in the strong coupling. The contributions of $\mathcal{O}\left(\alpha_{\mathrm{S}}^{2} \alpha^{2}\right), \mathcal{O}\left(\alpha_{\mathrm{S}} \alpha^{3}\right)$ and $\mathcal{O}\left(\alpha^{4}\right)$ will be denoted as LO, LO mix and LO EW, respectively. The two subleading orders contribute only via partonic channels with four external (anti)quark legs, and the LO EW contribution includes, inter alia, the production of dibosons with semi-leptonic decays. Representative Feynman diagrams for $V+2$ jet production are shown in figures 3 and 4 . The NLO contributions of $\mathcal{O}\left(\alpha_{\mathrm{S}}^{3} \alpha^{2}\right)$ and $\mathcal{O}\left(\alpha_{\mathrm{S}}^{2} \alpha^{3}\right)$ are denoted as NLO QCD and NLO EW, respectively. They are the main subject of this paper, while subleading NLO contributions of $\mathcal{O}\left(\alpha_{\mathrm{S}} \alpha^{4}\right)$ or $\mathcal{O}\left(\alpha^{5}\right)$ are not considered. Apart from the terminology, let us remind the reader that $\mathcal{O}\left(\alpha_{\mathrm{S}}^{2} \alpha^{3}\right)$ NLO EW contributions represent at the same time $\mathcal{O}(\alpha)$ corrections with respect to LO and $\mathcal{O}\left(\alpha_{\mathrm{S}}\right)$ corrections to LO mix contributions. Therefore, in order to cancel the $\mathcal{O}\left(\alpha_{\mathrm{S}}^{2} \alpha^{3}\right)$ leading logarithmic dependence on the renormalisation and factorization scales, NLO EW corrections should be combined with LO and LO mix terms. ${ }^{2}$

\footnotetext{
${ }^{2} \mathrm{LO}$ mix and NLO EW contributions are shown separately in the fixed-order analysis of section 4, while in the merging framework of section 5 they are systematically combined.
} 


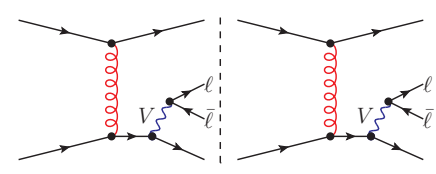

(a) $\mathcal{O}\left(\alpha_{\mathrm{S}}^{2} \alpha^{2}\right) \mathrm{LO}$

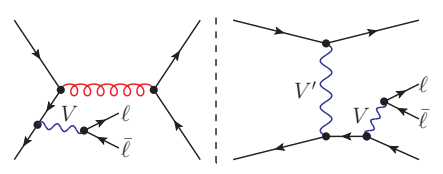

(b) $\mathcal{O}\left(\alpha_{\mathrm{S}} \alpha^{3}\right)$ LO mix

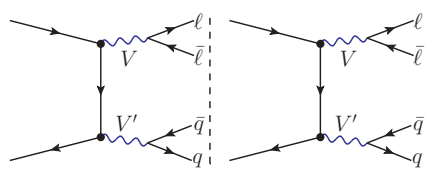

(c) $\mathcal{O}\left(\alpha^{4}\right)$ LO EW

Figure 3. Representative LO, LO mix and LO EW contributions to $V+2$ jet production.

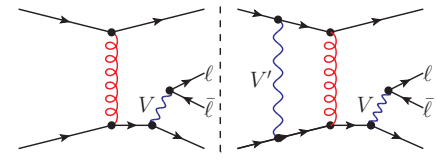

(a) Virtual $\mathcal{O}\left(\alpha_{\mathrm{S}}^{2} \alpha^{3}\right)$ correction

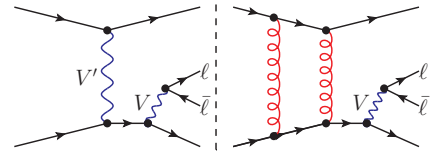

(b) Virtual $\mathcal{O}\left(\alpha_{\mathrm{S}}^{2} \alpha^{3}\right)$ correction

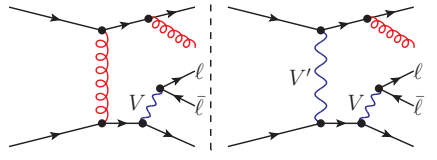

(c) Real $\mathcal{O}\left(\alpha_{\mathrm{S}}^{2} \alpha^{3}\right)$ correction

Figure 4. Representative virtual and real NLO EW contributions to $V+2$ jet production.

For what concerns the combination of NLO QCD and NLO EW corrections,

$$
\sigma_{\mathrm{QCD}}^{\mathrm{NLO}}=\sigma^{\mathrm{LO}}+\delta \sigma_{\mathrm{QCD}}^{\mathrm{NLO}}, \quad \sigma_{\mathrm{EW}}^{\mathrm{NLO}}=\sigma^{\mathrm{LO}}+\delta \sigma_{\mathrm{EW}}^{\mathrm{NLO}}
$$

as a default we adopt an additive prescription,

$$
\sigma_{\mathrm{QCD}+\mathrm{EW}}^{\mathrm{NLO}}=\sigma^{\mathrm{LO}}+\delta \sigma_{\mathrm{QCD}}^{\mathrm{NLO}}+\delta \sigma_{\mathrm{EW}}^{\mathrm{NLO}} .
$$

Here, for the case of $V+n$ jet production, $\sigma^{\mathrm{LO}}$ is the $\mathcal{O}\left(\alpha_{\mathrm{S}}^{n} \alpha^{2}\right)$ LO cross section, while $\delta \sigma_{\mathrm{QCD}}^{\mathrm{NLO}}$ and $\delta \sigma_{\mathrm{EW}}^{\mathrm{NLO}}$ correspond to the $\mathcal{O}\left(\alpha_{\mathrm{S}}^{n+1} \alpha^{2}\right)$ and $\mathcal{O}\left(\alpha_{\mathrm{S}}^{n} \alpha^{3}\right)$ corrections, respectively. Alternatively, in order to identify potentially large effects due to the interplay of EW and QCD corrections beyond NLO, we present results considering the following factorised combination of EW and QCD corrections,

$$
\sigma_{\mathrm{QCD} \times \mathrm{EW}}^{\mathrm{NLO}}=\sigma_{\mathrm{QCD}}^{\mathrm{NLO}}\left(1+\frac{\delta \sigma_{\mathrm{EW}}^{\mathrm{NLO}}}{\sigma^{\mathrm{LO}}}\right)=\sigma_{\mathrm{EW}}^{\mathrm{NLO}}\left(1+\frac{\delta \sigma_{\mathrm{QCD}}^{\mathrm{NLO}}}{\sigma^{\mathrm{LO}}}\right) .
$$

In situations where the factorised approach can be justified by a clear separation of scales - such as where QCD corrections are dominated by soft interactions well below the EW scale - the factorised formula (2.3) can be regarded as an improved prediction. However, in general, the difference between (2.2) and (2.3) should be considered as an estimate of unknown higher-order corrections of QCD-EW mixed type.

Subleading Born and photon-induced contributions of $\mathcal{O}\left(\alpha_{\mathrm{S}}^{n-1} \alpha^{3}\right)$ and $\mathcal{O}\left(\alpha_{\mathrm{S}}^{n-2} \alpha^{4}\right)$ will also be investigated and partly included in our predictions.

\subsection{Methods and tools}

Predictions presented in this paper have been obtained with the Monte Carlo frameworks Munich+OpenLoops and Sherpa+OpenLoops, which support in a fully automated way NLO QCD+EW simulations [41] at parton level and particle level, respectively. Virtual QCD and EW amplitudes are provided by the publicly ${ }^{3}$ available OpenLoops pro-

\footnotetext{
${ }^{3}$ The publicly available OpENLOOPS amplitude library includes all relevant matrix elements to compute NLO QCD corrections, including colour- and helicity-correlations and real radiation as well as loop-squared amplitudes, for more than a hundred LHC processes. Libraries containing NLO EW amplitudes will be provided soon.
} 
gram [46], which is based on a fast numerical recursion for the generation of one-loop scattering amplitudes [40]. Combined with the Colbien tensor reduction library [59], which implements the Denner-Dittmaier reduction techniques [60,61] and the scalar integrals of [62], the employed recursion permits to achieve very high CPU performance and a high degree of numerical stability. A sophisticated stability system is in place to rescue potential unstable phase-space points via a re-evaluation at quadrupole precision using CutTools [63], which implements the OPP method [64], together with the OnELOoP library [65]. As anticipated in the introduction, in order to address the production and decay of unstable particles, the original automation of one-loop EW corrections in OpenLoops [41] was supplemented by a fully general implementation of the complex-mass scheme [50].

All remaining tasks, i.e. the bookkeeping of partonic subprocesses, phase-space integration and the subtraction of QCD and QED bremsstrahlung are supported by the two independent and fully automated Monte Carlo generators Munich and Sherpa [47-49]. The first one, Munich, is a fully generic and very fast parton-level Monte Carlo integrator, which has been used, mainly in combination with OPENLOOPS, for various pioneering NLO multi-leg [66-69] and NNLO applications [70-75]. SHERPA is a particle-level Monte Carlo generator providing all stages of hadron collider simulations, including parton showering, hadronisation and underlying event simulations. It was used in the pioneering NLO QCD calculations of vector-boson plus multijet production [14-19], as well as for their matching to the parton shower [76] and the merging of multijet final states at NLO [54]. For tree amplitudes, with all relevant colour and helicity correlations, MUNich relies on OpenLoops, while Sherpa generates them internally with Amegic [77] and Comix [78]. For the cancellation of infrared singularities both Monte Carlo tools, Munich and SherPA, employ the dipole subtraction scheme $[79,80]$. Both codes were extensively checked against each other, and sub-permille level agreement was found.

\subsection{Physics objects and selection cuts}

For the definition of jets we employ the anti- $k_{\mathrm{T}}$ algorithm [81] with $R=0.4$. More precisely, in order to guarantee infrared safeness in presence of NLO QCD and EW corrections, we adopt a democratic clustering approach [82-84], where QCD partons and photons are recombined. In order to ensure the cancellation of collinear singularities that arise from collinear photon emissions off charged leptons and quarks, collinear pairs of photons and charged fermions with $\Delta R_{\gamma f}<0.1$ are recombined via four-momentum addition, and all observables are defined in terms of such dressed fermions. Fermion dressing is applied prior to the jet algorithm, and photons that have been recombined with leptons, as well as (dressed) leptons, are not subject to jet clustering.

After jet clustering QCD jets are separated from photons by imposing an upper bound $z_{\text {thr }}=0.5$ to the photon energy fraction inside jets. In this case, the cut $z_{\text {thr }}<0.5$ is applied only to photons that are inside the jet, but outside the technical recombination cone with $\Delta R_{\gamma q}<0.1$. The recombination of (anti)quark-photon pairs with $\Delta R_{\gamma q}<0.1$ represents a technical regularisation prescription to ensure the cancellation of collinear photon-quark singularities. As demonstrated in [41], this provides an excellent approxi- 


\begin{tabular}{|lc|c|c|c|}
\hline & & $\mathrm{W}^{ \pm} \rightarrow \ell^{+} \nu_{\ell}, \ell^{-} \bar{\nu}_{\ell}$ & $\mathrm{Z} \rightarrow \ell^{+} \ell^{-}$ & $\mathrm{Z} \rightarrow \nu_{\ell} \bar{\nu}_{\ell}$ \\
\hline$\ell$ & $\in$ & $e, \mu$ & $e, \mu$ & $e, \mu, \tau$ \\
$p_{\mathrm{T}, \ell^{ \pm}}[\mathrm{GeV}]$ & $>$ & 25 & 25 & \\
$E_{\mathrm{T}}[\mathrm{GeV}]$ & $>$ & 25 & & 25 \\
$m_{\mathrm{T}}^{\mathrm{W}}[\mathrm{GeV}]$ & $>$ & 40 & & \\
$m_{\ell^{+} \ell^{-}}[\mathrm{GeV}]$ & $\in$ & & {$[66,116]$} & \\
$\left|\eta_{\ell^{ \pm}}\right|$ & $<$ & 2.5 & 2.5 & \\
$\Delta R_{\ell^{ \pm} j}$ & $>$ & 0.5 & 0.5 & \\
$\Delta R_{\ell^{+} \ell^{-}}$ & $>$ & & 0.2 & \\
\hline
\end{tabular}

Table 1. Selection cuts for the various $V+$ jets production and decay processes. The missing transverse energy $E_{\mathrm{T}}$ is calculated from the vector sum of neutrino momenta, and the W-boson transverse mass is defined as $m_{\mathrm{T}}^{\mathrm{W}}=\sqrt{2 p_{\mathrm{T}, \ell} p_{\mathrm{T}, \nu}\left(1-\cos \Delta \phi_{\ell \nu}\right)}$. The lepton-jet separation cut, $\Delta R_{\ell^{ \pm} j}>0.5$ is applied to all jets in the region (2.4).

mation to a more rigorous approach for the cancellation of collinear singularities based on fragmentation functions.

For the selection of signatures of type $\ell \ell / \ell \nu / \nu \nu+1,2$ jets, which result from the various vector-boson decays, we apply the leptonic cuts listed in table 1 . They correspond to the ATLAS analysis of [85].

Events will be categorised according to the number of anti- $k_{\mathrm{T}}$ jets in the transversemomentum and pseudo-rapidity region

$$
p_{\mathrm{T}, j}>30 \mathrm{GeV}, \quad\left|\eta_{j}\right|<4.5 .
$$

Additionally, for certain observables we present results vetoing a second jet with details explained in the text.

\subsection{Input parameters, scale choices and variations}

As input parameters to simulate $p p \rightarrow \ell \ell / \ell \nu / \nu \nu+$ jets at NLO QCD+EW we use the gauge-boson masses and widths [86]

$$
M_{\mathrm{Z}}=91.1876 \mathrm{GeV}, \quad M_{\mathrm{W}}=80.385 \mathrm{GeV}, \quad \Gamma_{\mathrm{Z}}=2.4955 \mathrm{GeV}, \quad \Gamma_{\mathrm{W}}=2.0897 \mathrm{GeV} .
$$

The latter are obtained from state-of-the art theoretical calculations. For the top quark we use the mass reported in [86], and we compute the width at NLO QCD,

$$
m_{\mathrm{t}}=173.2 \mathrm{GeV}, \quad \Gamma_{\mathrm{t}}=1.339 \mathrm{GeV} .
$$

For the Higgs-boson mass and width [87] we use

$$
M_{\mathrm{H}}=125 \mathrm{GeV}, \quad \Gamma_{\mathrm{H}}=4.07 \mathrm{MeV} .
$$

Electroweak contributions to $p p \rightarrow V+2$ jets involve topologies with $s$-channel top-quark and Higgs propagators that require a finite top and Higgs width. However, at the perturbative order considered in this paper, such topologies arise only in interference terms that 
do not give rise to Breit-Wigner resonances. The dependence of our results on $\Gamma_{t}$ and $\Gamma_{H}$ is thus completely negligible.

All unstable particles are treated in the complex-mass scheme [50], where width effects are absorbed into the complex-valued renormalised masses

$$
\mu_{i}^{2}=M_{i}^{2}-\mathrm{i} \Gamma_{i} M_{i} \quad \text { for } i=\mathrm{W}, \mathrm{Z}, \mathrm{t}, \mathrm{H} .
$$

The electroweak couplings are derived from the gauge-boson masses and the Fermi constant, $G_{\mu}=1.16637 \times 10^{-5} \mathrm{GeV}^{-2}$, using

$$
\alpha=\left|\frac{\sqrt{2} s_{\mathrm{w}}^{2} \mu_{\mathrm{W}}^{2} G_{\mu}}{\pi}\right|,
$$

where the W-boson mass and the squared sine of the mixing angle,

$$
s_{\mathrm{w}}^{2}=1-c_{\mathrm{w}}^{2}=1-\frac{\mu_{\mathrm{W}}^{2}}{\mu_{\mathrm{Z}}^{2}},
$$

are complex-valued. The $G_{\mu}$-scheme guarantees an optimal description of pure $\mathrm{SU}(2)$ interactions at the electroweak scale. It is the scheme of choice for $\mathrm{W}+$ jets production, and it provides a very decent description of $\mathrm{Z}+$ jets production as well.

The CKM matrix is assumed to be diagonal, while colour effects and related interferences are included throughout, without applying any large- $N_{c}$ expansion.

For the calculation of hadron-level cross sections we employ the NNPDF2.3 QED parton distributions [88] which include NLO QCD and LO QED effects, and we use the PDF set corresponding to $\alpha_{\mathrm{S}}\left(M_{\mathrm{Z}}\right)=0.118 .{ }^{4}$ Matrix elements are evaluated using the running strong coupling supported by the PDFs, and, consistently with the variable flavournumber scheme implemented in the NNPDFs, at the top threshold we switch from five to six active quark flavours in the renormalisation of $\alpha_{\mathrm{S}}$. All light quarks, including bottom quarks, are treated as massless particles, and top-quark loops are included throughout in the calculation. The NLO PDF set is used for LO as well as for NLO QCD and NLO EW predictions.

In all fixed-order results the renormalisation scale $\mu_{R}$ and factorisation scale $\mu_{F}$ are set to

$$
\mu_{\mathrm{R}, \mathrm{F}}=\xi_{\mathrm{R}, \mathrm{F}} \mu_{0}, \quad \text { with } \quad \mu_{0}=\hat{H}_{\mathrm{T}}^{\prime} / 2 \quad \text { and } \quad \frac{1}{2} \leq \xi_{\mathrm{R}}, \xi_{\mathrm{F}} \leq 2
$$

where $\hat{H}_{\mathrm{T}}^{\prime}$ is the scalar sum of the transverse energy of all parton-level final-state objects,

$$
\hat{H}_{\mathrm{T}}^{\prime}=\sum_{i \in\{\text { quarks,gluons }\}} p_{\mathrm{T}, i}+p_{\mathrm{T}, \gamma}+E_{\mathrm{T}, V}
$$

Also QCD partons and photons that are radiated at NLO are included in $\hat{H}_{\mathrm{T}}^{\prime}$, and the vector-boson transverse energy, $E_{\mathrm{T}, V}$, is computed using the total (off-shell) fourmomentum of the corresponding decay products, i.e.

$$
E_{\mathrm{T}, \mathrm{Z}}^{2}=p_{\mathrm{T}, \ell \ell}^{2}+m_{\ell \ell}^{2}, \quad E_{\mathrm{T}, \mathrm{W}}^{2}=p_{\mathrm{T}, \ell \nu}^{2}+m_{\ell \nu}^{2} .
$$

\footnotetext{
${ }^{4}$ To be precise we use the NNPDF23_nlo_as_0118_qed set interfaced through the LHAPDF library 5.9.1 (Munich) and 6.1.5 (Sherpa) [89].
} 
In order to guarantee infrared safeness at NLO EW, the scale (2.12) must be insensitive to collinear photon emissions off quarks and leptons. To this end, all terms in (2.12)-(2.13) are computed in terms of dressed leptons and quarks, while the $p_{\mathrm{T}, \gamma}$ term in (2.12) involves only photons that have not been recombined with charged fermions.

Our default scale choice corresponds to $\xi_{\mathrm{R}}=\xi_{\mathrm{F}}=1$, and theoretical fixed-order uncertainties are assessed by applying the scale variations $\left(\xi_{\mathrm{R}}, \xi_{\mathrm{F}}\right)=(2,2),(2,1),(1,2)$, $(1,1),(1,0.5),(0.5,1),(0.5,0.5)$, while theoretical uncertainties of our MEPs predictions are assessed by applying the scale variations $\left(\xi_{\mathrm{R}}, \xi_{\mathrm{F}}\right)=(2,2),(1,1),(0.5,0.5)$. As shown in [1419] the scale choice (2.11) guarantees a good perturbative convergence for $V+$ multijet production over a wide range of observables and energy scales.

\section{Giant $K$-factors and electroweak corrections for $V+1$ jet production}

In this section we start our discussion of $V+$ jets production at NLO QCD + EW by recalling some pathological features of fixed-order calculations for $p p \rightarrow V+1$ jet. Such observations will provide the main motivation for the multiparticle calculations and the multijet merging approach presented in sections 4 and 5 .

It is well known that NLO QCD predictions for $V+1$ jet production $[7-9,34]$ suffer from a very poor convergence of the perturbative expansion, which manifests itself in the form of giant $K$-factors [51] at large jet transverse momenta. In this kinematic regime the NLO QCD cross section is dominated by dijet configurations where the hardest jet recoils against a similarly hard second jet, while the vector boson remains relatively soft. Such bremsstrahlung configurations are effectively described at LO, with correspondingly large scale uncertainties. Moreover, in this situation NLO EW calculations for $p p \rightarrow V+1$ jet are meaningless, as they completely miss EW correction effects for the dominating dijet configurations.

The above mentioned anomalies are clearly manifest in figure 5, where NLO QCD and EW effects in $p p \rightarrow \ell^{-} \bar{\nu}_{\ell}+1$ jet $^{5}$ are plotted versus the transverse momenta of the reconstructed vector boson, defined in terms of their decay products, i.e. $p_{\mathrm{T}, \mathrm{V}}=\mid \vec{p}_{\mathrm{T}, \ell_{1}}+$ $\vec{p}_{\mathrm{T}, \ell_{2}} \mid$ for $V \rightarrow \ell_{1} \ell_{2}$, and of the leading jet. While overall QCD corrections to the W-boson $p_{\mathrm{T}}$ distribution are moderate (at the level of 40-50\%) they strongly increase in the tail of the distribution reaching $100 \%$ at $3 \mathrm{TeV}$. In the case of the jet- $p_{\mathrm{T}}$ the $\mathrm{QCD}$ corrections show a clear pathological behaviour growing larger than several $100 \%$ in the multi-TeV region. In the $p_{\mathrm{T}, \mathrm{W}}$ distribution, NLO EW corrections present a consistent Sudakov shape, with corrections growing negative like $\ln ^{2}\left(\hat{s} / M_{\mathrm{W}}^{2}\right)$ and reaching a few tens of percent in the tail. However, as reflected in the sizeable disparity between additive QCD+EW and multiplicative $\mathrm{QCD} \times \mathrm{EW}$ combinations, the large size of NLO QCD and NLO EW effects suggests the presence of important uncontrolled mixed NNLO QCD-EW corrections. In the case of the jet- $p_{\mathrm{T}}$ distribution these problems become dramatic. Besides the explosion of NLO QCD corrections, in the multi-TeV range we observe a pathological NLO EW

\footnotetext{
${ }^{5} \mathrm{~A}$ similar behaviour is encountered also in the various other channels with $\ell \ell / \ell \nu / \nu \nu+$ jet final states. A more detailed discussion of the interplay between QCD and EW corrections in the presence of giant $K$-factors, for the case of $\mathrm{W}+$ jets production, can be found in section 6.1 of [41].
} 
behaviour, with large positive corrections instead of negative Sudakov effects. On one side, similarly as for the giant QCD $K$-factor, this feature can be attributed to hard dijet configurations that enter the NLO EW bremsstrahlung through mixed QCD-EW terms of $\mathcal{O}\left(\alpha_{\mathrm{S}} \alpha^{3}\right)$ (see figure 2(c)). On the other side, EW Sudakov effects are completely suppressed due to the absence of one-loop corrections for $V+2$ jet configurations.

In principle, the pathological behaviour of NLO predictions can be avoided by imposing a cut that renders the $V+1$ jet cross section sufficiently exclusive with respect to extra jet radiation. For instance, as shown in the right plot of figure 5, suppressing bremsstrahlung effects with a veto against dijet configurations with angular separation $\Delta \phi_{j_{1} j_{2}}>3 \pi / 4$ leads to well-behaved QCD predictions and a standard NLO EW Sudakov behaviour, with up to $-40 \%$ corrections at $p_{\mathrm{T}}=2 \mathrm{TeV}$.

Thus, giant $K$-factors and related issues can be circumvented through a jet veto. However, in order to obtain a precise theoretical description of inclusive $V+$ jets production at $\operatorname{high} p_{\mathrm{T}}$, it is clear that fixed-order NLO QCD+EW calculations for one-jet final states have to be supplemented by corresponding predictions for multijet final states. This task, as well as the consistent merging of NLO QCD+EW cross sections with different jet multiplicity, will be the subject of the rest of this paper.

\section{Fixed-order predictions for $V+2$ jet production}

In this section we present numerical results for $\ell \ell / \ell \nu / \nu \nu+2$ jet production, including NLO QCD and EW corrections, as well as subleading Born and photon-induced contributions.

\subsection{NLO QCD+EW predictions}

In the following, we discuss a series of fixed-order NLO QCD+EW results for $p p \rightarrow V+2$ jets including leptonic decays, i.e. we investigate the processes $p p \rightarrow \ell^{+} \nu_{\ell}+2$ jets, $p p \rightarrow \ell^{-} \bar{\nu}_{\ell}+$ 2 jets, $p p \rightarrow \ell^{+} \ell^{-}+2$ jets and $p p \rightarrow \nu_{\ell} \bar{\nu}_{\ell}+2$ jets at $13 \mathrm{TeV}$. We will focus on the effect of EW corrections on the $p_{\mathrm{T}}$ spectra of reconstructed vector bosons, charged leptons and jets. Such observables are of direct relevance as a background for many searches for new physics including dark matter at the LHC. Instead of presenting the four processes and their higher-order corrections independently, we will mostly show them together for the different observables in order to highlight important similarities and investigate possible differences. Additionally, for $p p \rightarrow \ell \nu+2$ jets we show distributions in the transverse mass and missing energy, while for $p p \rightarrow \ell^{+} \ell^{-}+2$ jets we show the distribution in the invariant mass of the leptonic decay products. Predictions for further kinematic observables are presented in appendix A. ${ }^{6}$

Figure 6 displays results for the transverse-momentum spectra of the reconstructed (offshell) vector bosons. For all processes NLO QCD corrections are remarkably small, and even in the tails scale uncertainties hardly exceed $10 \%$. In contrast, NLO EW corrections feature a standard Sudakov behaviour and become very large at high $p_{\mathrm{T}}$. They exceed QCD

\footnotetext{
${ }^{6}$ Our NLO EW predictions for $p p \rightarrow \ell^{+} \ell^{-}+2$ jets have been compared in detail against the results of [45]. Good agreement was found within the small uncertainties due to the different treatment of photons and b-quark induced processes.
} 

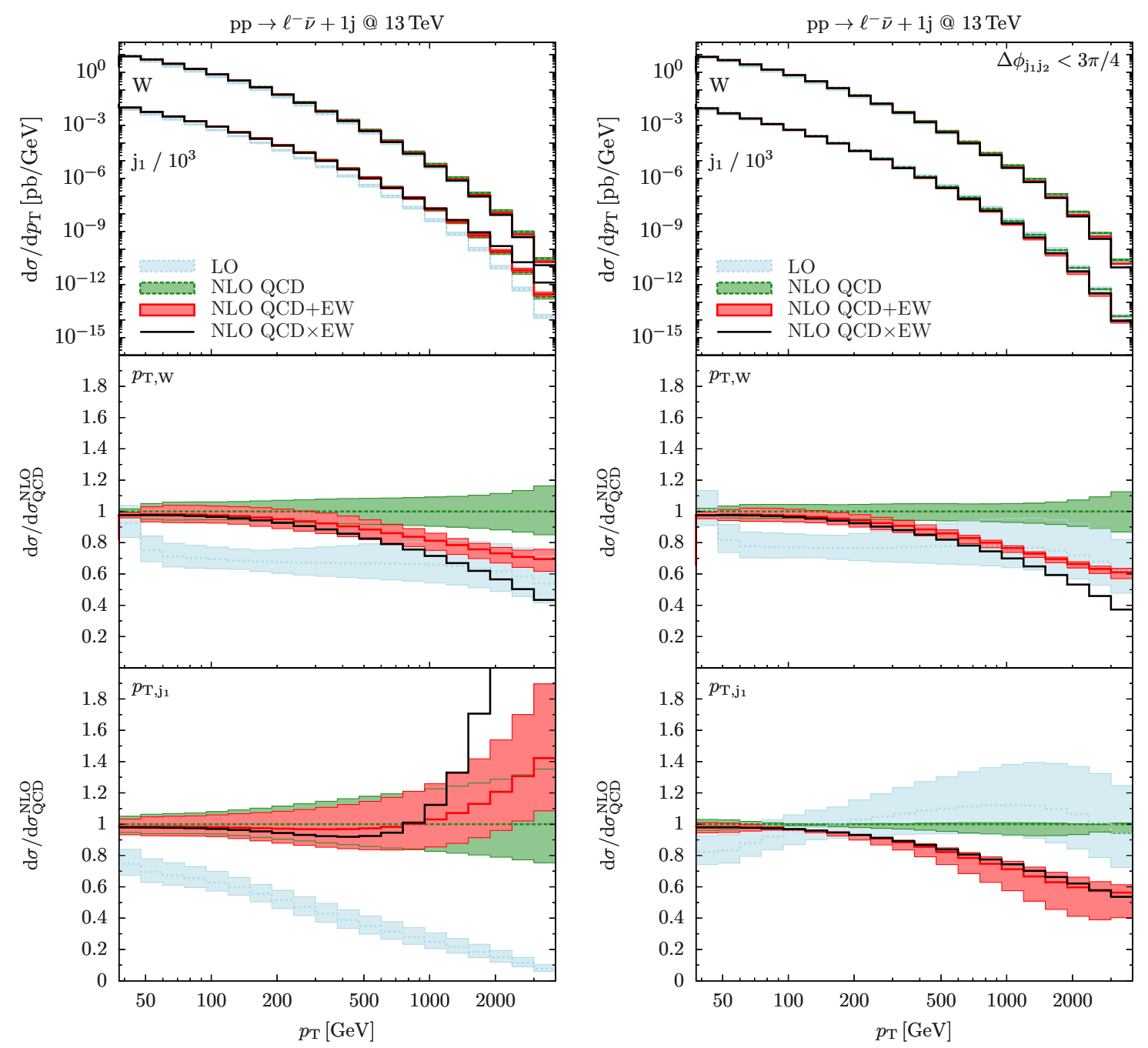

Figure 5. Distributions in the transverse momenta of the reconstructed vector boson, $p_{\mathrm{T}, \mathrm{V}}$, and of the hardest jet, $p_{\mathrm{T}, j_{1}}$, for $p p \rightarrow \ell^{-} \bar{\nu}_{\ell}+1$ jet at $13 \mathrm{TeV}$ with standard cuts (left) and with an additional cut $\Delta \phi_{j_{1} j_{2}}<3 \pi / 4$ (right). Absolute LO (light blue), NLO QCD (green), NLO QCD+EW (red) and NLO $\mathrm{QCD} \times \mathrm{EW}$ (black) predictions (upper panel) and relative corrections with respect to NLO QCD (lower panels). The bands correspond to scale variations, and in the case of ratios only the numerator is varied. The absolute predictions in $p_{\mathrm{T}, j_{1}}$ are rescaled by a factor $10^{-3}$.

scale uncertainties already at a few hundred $\mathrm{GeV}$ and reach about $-40 \%$ at $2 \mathrm{TeV}$. Due to the small size of QCD corrections, for all processes we observe a good consistency between $\mathrm{NLO} \mathrm{QCD}+\mathrm{EW}$ and $\mathrm{NLO} \mathrm{QCD} \times \mathrm{EW}$ results. As expected, $\mathrm{QCD}$ and $\mathrm{EW}$ corrections for $\ell^{+} \nu_{\ell}+2$ jets turn out to be very similar to the ones observed in the corresponding calculation of [41] where the $\mathrm{W}$ boson was kept on-shell.

In figure 7 we plot, where applicable, the $p_{\mathrm{T}}$ spectra of the hardest lepton. The behaviour of the QCD and EW corrections is very similar to the one observed for the $p_{\mathrm{T}}$ of the reconstructed vector bosons. Clearly, the observed large Sudakov corrections are a result of the $\mathrm{TeV}$ scale dynamics that enter the production of a high- $p_{\mathrm{T}}$ vector boson, while 

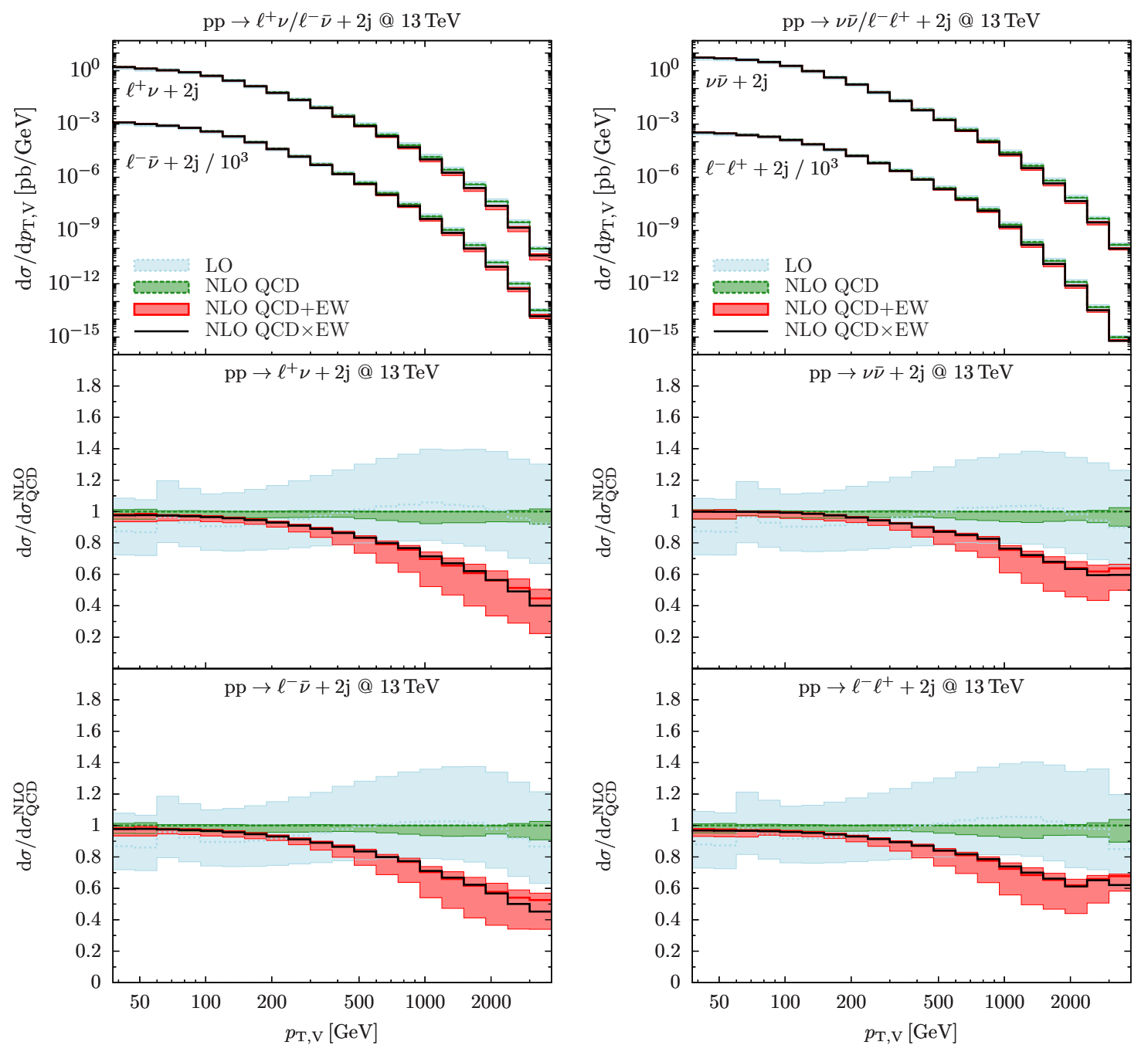

Figure 6. Distributions in the reconstructed transverse momentum of the off-shell vector boson, $p_{\mathrm{T}, \mathrm{V}}$, for $p p \rightarrow \ell \nu+2$ jets (left) and $p p \rightarrow \ell \ell / \nu \nu+2$ jets (right) at $13 \mathrm{TeV}$. Curves and bands as in figure 5 .

they are hardly affected by vector-boson decay processes, which occur at much smaller energy scales.

Figures 8 and 9 present distributions in the transverse momenta of the hardest and second-hardest jet, respectively. Again, the perturbative QCD expansion turns out to be very stable, with scale uncertainties that hardly exceed $10 \%$. In these jet- $p_{\mathrm{T}}$ distributions we observe smaller NLO EW corrections as compared to the case of the vector-boson $p_{\mathrm{T}}$ spectrum. This is due to the fact that $W$ and $Z$ bosons carry larger $\mathrm{SU}(2)$ charges as compared to gluons and quarks inside jets. Thus, the largest EW Sudakov corrections arise when the vector-boson $p_{\mathrm{T}}$ is highest, while very hard jets in combination with less hard vector bosons yield less pronounced EW Sudakov logarithms. We also find that, at a given $p_{\mathrm{T}}$, the second jet always receives larger $\mathrm{EW}$ corrections than the first jet. Quantitatively, the EW corrections to the different $\mathrm{W}+2$ jet and $Z+2$ jet processes are 


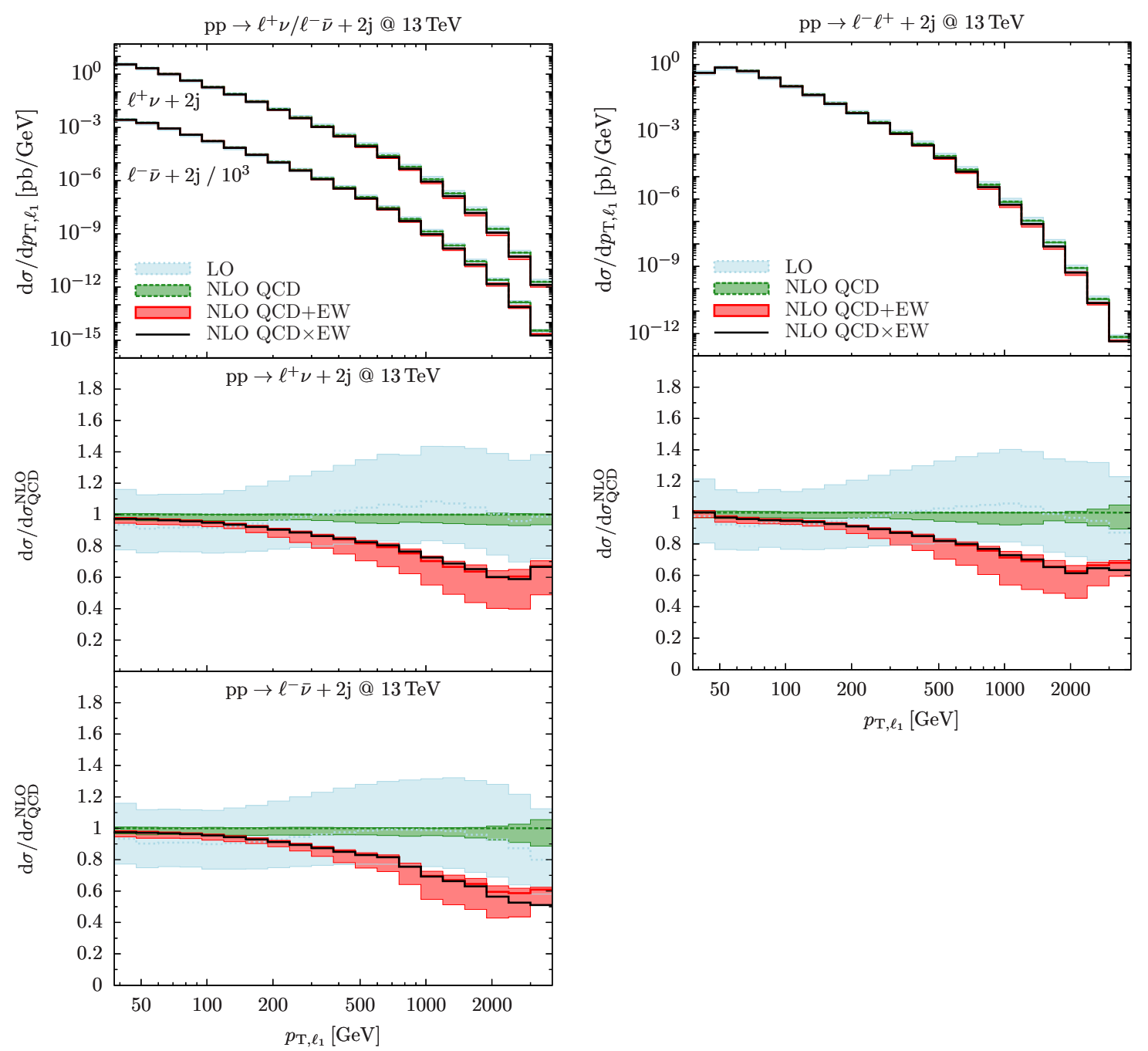

Figure 7. Distributions in the transverse momentum of the hardest charged lepton, $p_{\mathrm{T}, \ell_{1}}$, for $p p \rightarrow \ell \nu+2$ jets (left) and $p p \rightarrow \ell \ell+2$ jets (right) at $13 \mathrm{TeV}$. Curves and bands as in figure 5 .

rather similar. Thus, corresponding ratios are expected to be only mildly sensitive to EW (or QCD) corrections.

In figure 10 we show distributions in the transverse mass, $m_{\mathrm{T}}^{\mathrm{W}}$, and in the missing transverse energy (i.e. the $p_{\mathrm{T}}$ spectrum of the neutrino) for $\mathrm{W}+2$ jet production. Both observables are of paramount importance in many BSM searches, especially in the highenergy regime. Again, QCD effects and uncertainties turn out to be rather mild. As far as EW corrections are concerned, at large transverse masses we observe only a minor impact, which does not exceed $-10 \%$ and remains at the level of QCD scale uncertainties. In contrast, and as expected, the missing-energy distributions follow the behaviour of the lepton- $p_{\mathrm{T}}$ distribution shown in figure 7 , and NLO EW corrections reach about $-40 \%$ at $2 \mathrm{TeV}$.

Finally, in figure 11 we turn to the differential distribution in the invariant mass, $m_{\ell \ell}$, of the lepton pair produced in $p p \rightarrow \ell^{+} \ell^{-}+2$ jets. The plotted range corresponds to the 

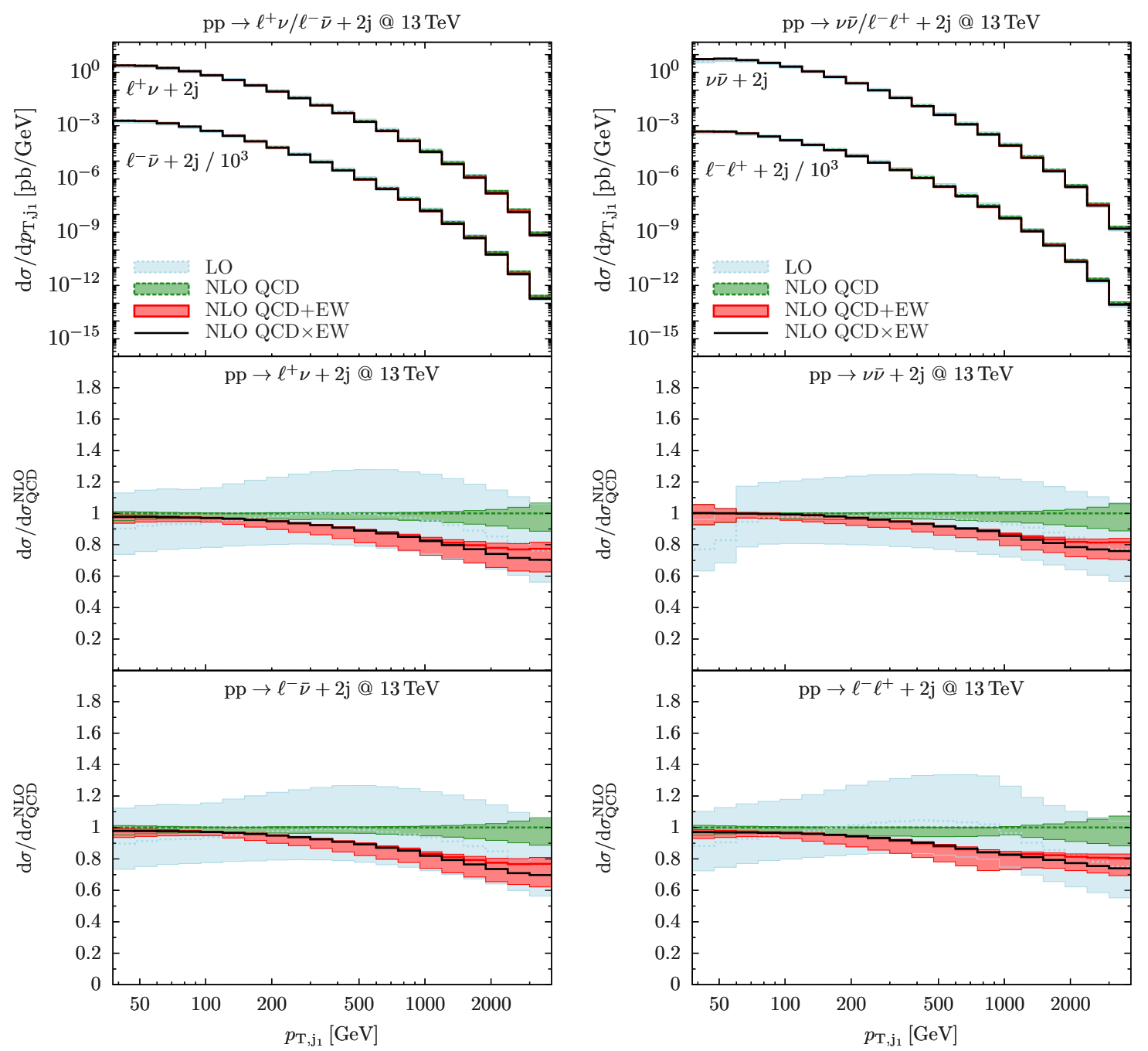

Figure 8. Distributions in the transverse momentum of the hardest jet, $p_{\mathrm{T}, j_{1}}$, for $p p \rightarrow \ell \nu+2$ jets (left) and $p p \rightarrow \ell \ell / \nu \nu+2$ jets (right) at $13 \mathrm{TeV}$. Curves and bands as in figure 5 .

event selection specified in table 1 and does not extend up to the high-energy region, where EW Sudakov effects would show up. However, the NLO EW corrections are sensitive to QED radiation off the charged leptons and shift parts of the cross section from above the Breit-Wigner peak to below the peak. The observed shape of the EW corrections is qualitatively very similar to the well-known NLO EW corrections to neutral-current DrellYan production [90, 91]. In this kinematic regime, QCD corrections are very small and always below $10 \%$, while scale uncertainties are as small as a few percent.

In summary, NLO QCD+EW effects for $p p \rightarrow V+2$ jets turn out to be completely free from the perturbative instabilities that plague NLO predictions for $V+1$ jet production: the perturbative QCD expansion is very well behaved, and NLO EW corrections feature, as expected, Sudakov effects that become very large at the TeV scale, especially for $V+$ 2 jet configurations where the highest transverse momentum is carried by the electroweak vector boson. 

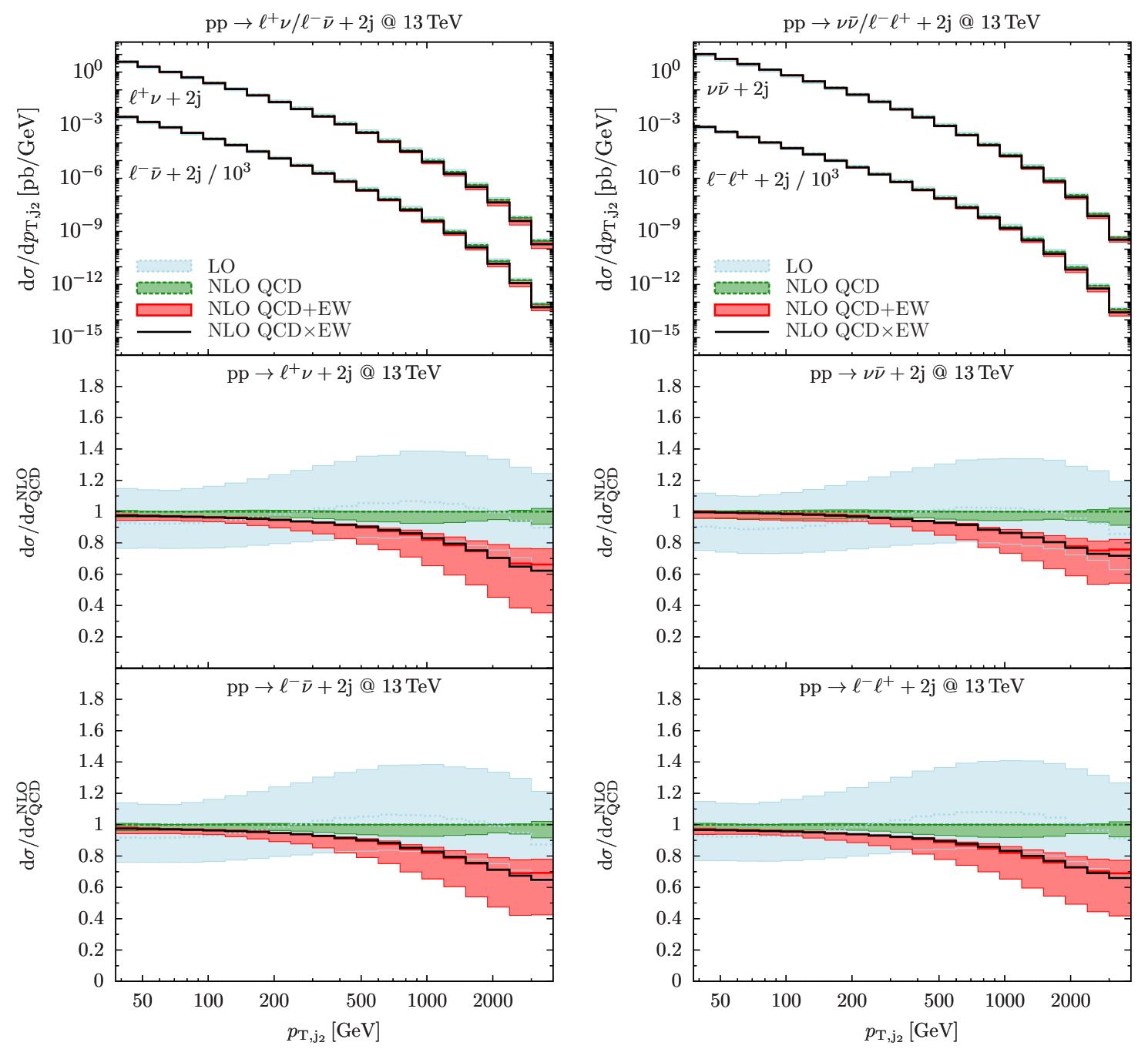

Figure 9. Distributions in the transverse momentum of the second-hardest jet, $p_{\mathrm{T}, j_{2}}$, for $p p \rightarrow$ $\ell \nu+2$ jets (left) and $p p \rightarrow \ell \ell / \nu \nu+2$ jets (right) at $13 \mathrm{TeV}$. Curves and bands as in figure 5 .

\subsection{Subleading Born and photon-induced contributions}

In this section we quantify the numerical impact of subleading Born and photon-induced ( $p \gamma$ and $\gamma \gamma$ initial states) contributions to $V+2$ jet production with leptonic decays, i.e. tree-level contributions of $\mathcal{O}\left(\alpha_{\mathrm{S}} \alpha^{3}\right)$ and $\mathcal{O}\left(\alpha^{4}\right) .{ }^{7}$

Figures 12 and 13 illustrate the subleading contributions for the distributions in the transverse momenta of the reconstructed vector-boson and of the hardest jet, respectively. Although mostly suppressed by several orders of magnitude at small energies, at large energies $p \gamma$-initiated production can have a sizable impact on the $p_{\mathrm{T}}$ spectrum of the vector boson, whereas the LO mix contribution grows up to several tens of percent in the

\footnotetext{
${ }^{7}$ The subleading Born contributions of $\mathcal{O}\left(\alpha^{4}\right)$ are dominated by diboson production with semi-leptonic decays. In order to avoid a double counting between diboson and $V+$ jets processes we do not include those contributions in any of our predictions in the following sections.
} 

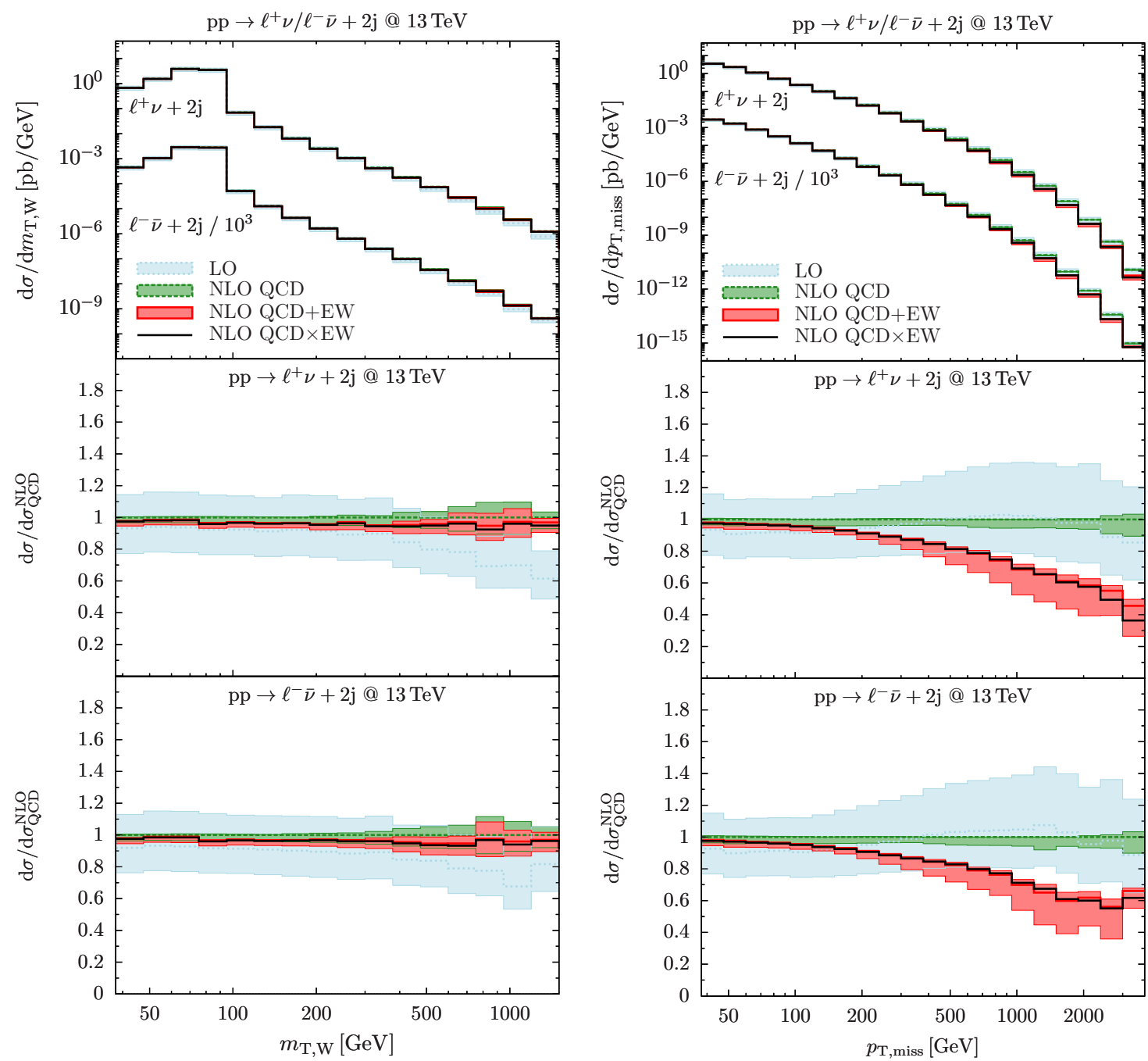

Figure 10. Distributions in the transverse mass, $m_{\mathrm{T}}^{\mathrm{W}}=\sqrt{2 p_{\mathrm{T}, \ell} p_{\mathrm{T}, \nu}\left(1-\cos \Delta \phi_{\ell \nu}\right)}$, (left) and the missing transverse energy, $p_{\mathrm{T} \text {,miss }}$, (right) for $p p \rightarrow \ell \nu+2$ jets at $13 \mathrm{TeV}$. Curves and bands as in figure 5 .

multi- $\mathrm{TeV}$ region of the jet- $p_{\mathrm{T}}$ spectrum. These effects can both be understood as induced by PDFs: in current PDF fits including QED corrections [88] the photon density at high Bjoerken $x$ strongly increases, while at the same time a relative increase of quark PDFs over the gluon PDF induces an enhancement of the four-quark channel (which involves LO mix terms) over the two-quark channel. Although strongly suppressed in the full $p_{\mathrm{T}}$ range, it is interesting to note that the LO mix contributions to the $p_{\mathrm{T}}$ spectrum of the reconstructed vector bosons feature a different behaviour in the case of $\ell^{+} \nu j j$ vs. $\ell^{-} \bar{\nu} j j$ and $\ell^{+} \ell^{-} j j$ vs. $\nu \bar{\nu} j j$ production (see figure 12). In all cases we observe a sign flip that results from the interference of resonant EW diagrams with non-resonant QCD amplitudes (see the discussion of "pseudo resonances" in [41]). However, the location of the sign flip and the subsequent onset of a sizable negative contribution is significantly displaced in the different related processes. This can be attributed to the fact that the position of the sign flip is 


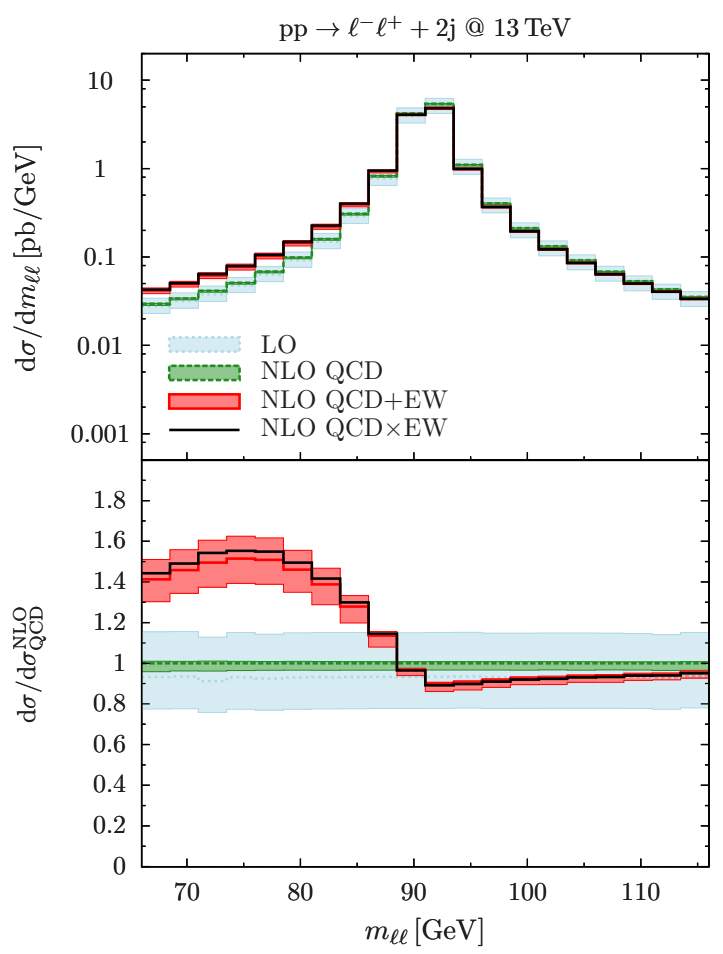

Figure 11. Distribution in the invariant mass $m_{\ell \ell}$ of the lepton pair in $p p \rightarrow \ell^{+} \ell^{-}+2$ jet production at $13 \mathrm{TeV}$. Curves and bands as in figure 5 .

very sensitive to phase-space boundaries and the relative yields of the various contributing partonic channels, which in turn is sensitive to differences in the PDF luminosities that enter the various processes.

With respect to the large impact of the $p \gamma$-initiated production at large vector-boson $p_{\mathrm{T}}$, one should, however, keep in mind that the photon PDF is still very poorly constrained in this regime [88]. Therefore, we do not include these contributions in any of the predictions for $V+$ jets production in the rest of the paper.

Having a merging of different jet multiplicities in mind, we want to note that the LO mix contributions to $p p \rightarrow V+2$ jet production discussed here are in fact identical with the QCD-EW mixed bremsstrahlung contributions to $p p \rightarrow V+1$ jet production. The multijet merging approach introduced in the next section guarantees a consistent inclusion of such effects without double counting.

\section{Multijet merged predictions for $V+$ jets at NLO QCD+EW}

In order to address the need of NLO QCD+EW accuracy for observables that receive sizable contributions from multijet radiation, in this section we introduce an approach that allows one to readily implement $\mathrm{NLO} \mathrm{QCD}+\mathrm{EW}$ effects in the context of multijet merging. The benefits of multijet merging are first illustrated through a naïve combination of fixed-order calculations for $V+1$ jet and $V+2$ jet production based on exclusive sums. Subsequently, we introduce an approximate treatment of EW corrections, based on infrared-subtracted virtual contributions, which allows us to include EW corrections in the MEPs@NLO multi- 

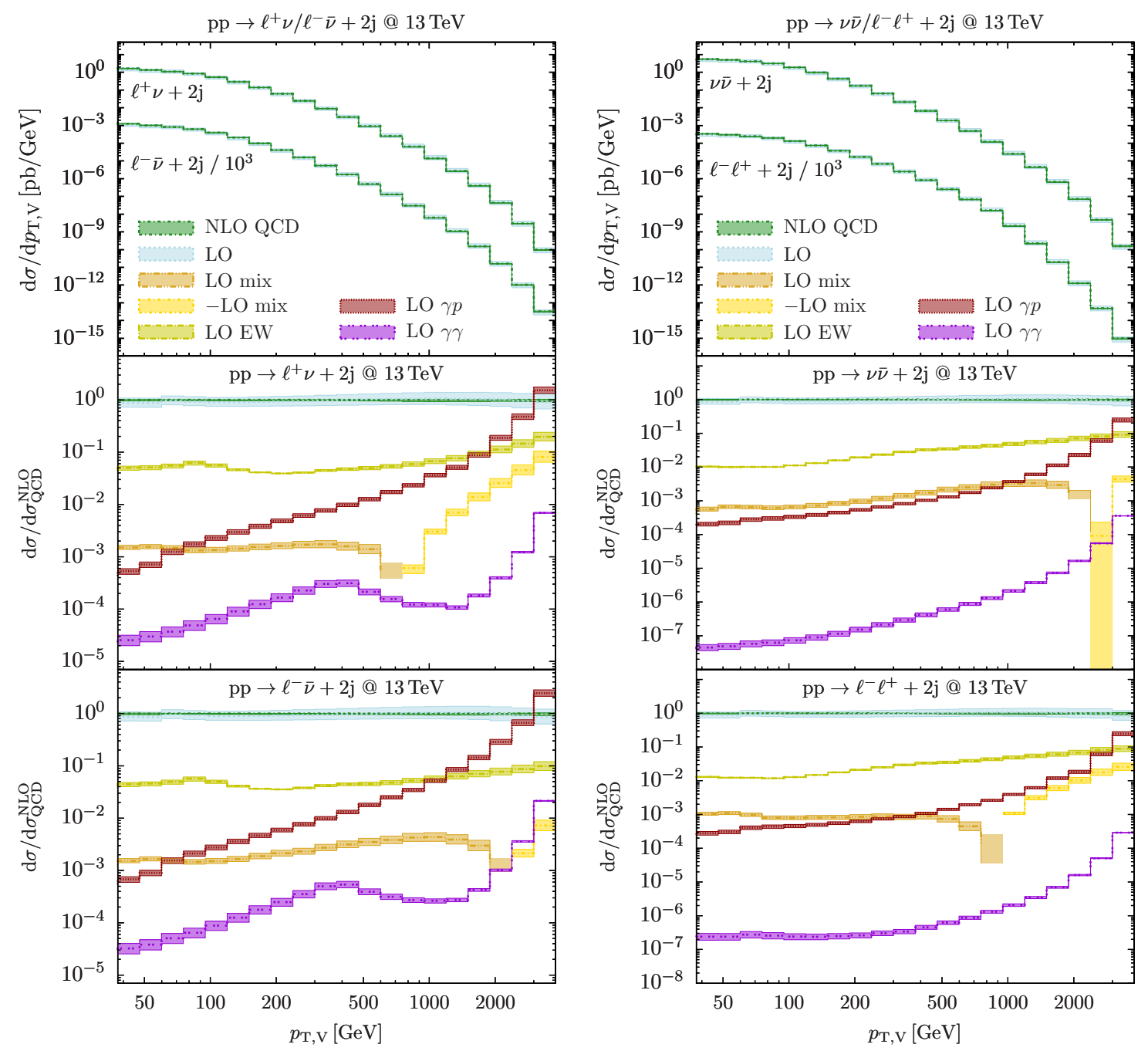

Figure 12. Distributions in the reconstructed transverse momenta of the vector boson for off-shell $\mathrm{W}^{ \pm}+2$ jet (left) and $\mathrm{Z}+2$ jet (right) production and decay. Absolute LO (light blue) and NLO QCD (green) predictions (upper panel) and relative corrections with respect to NLO QCD, showing subleading Born contributions (lower panels). Discontinuities indicate sign changes of the LO mix contribution. The bands correspond to scale variations, and in the case of ratios only the numerator is varied. The absolute predictions for $p p \rightarrow \ell^{-} \bar{\nu}_{\ell}+2$ jets and $p p \rightarrow \ell^{+} \ell^{-}+2$ jets are rescaled by a factor $10^{-3}$.

jet merging framework [54, 92] in a rather straightforward way. Finally, based on a fully automated implementation of this approach in SHERPA+OPEnLoOPs, we present an inclusive simulation of vector-boson plus multijet production that provides NLO QCD+EW accuracy for $V+0,1,2$ jet final states.

\subsection{Combining $p p \rightarrow V+1,2$ jets with exclusive sums}

From the discussion of giant $K$-factors in section 3 it should be clear that a theoretically well behaved and phenomenologically sensible prediction of inclusive $V+$ jets cross sections can only be achieved combining NLO QCD + EW cross sections for $V+1$ jet and $V+$ multijet 

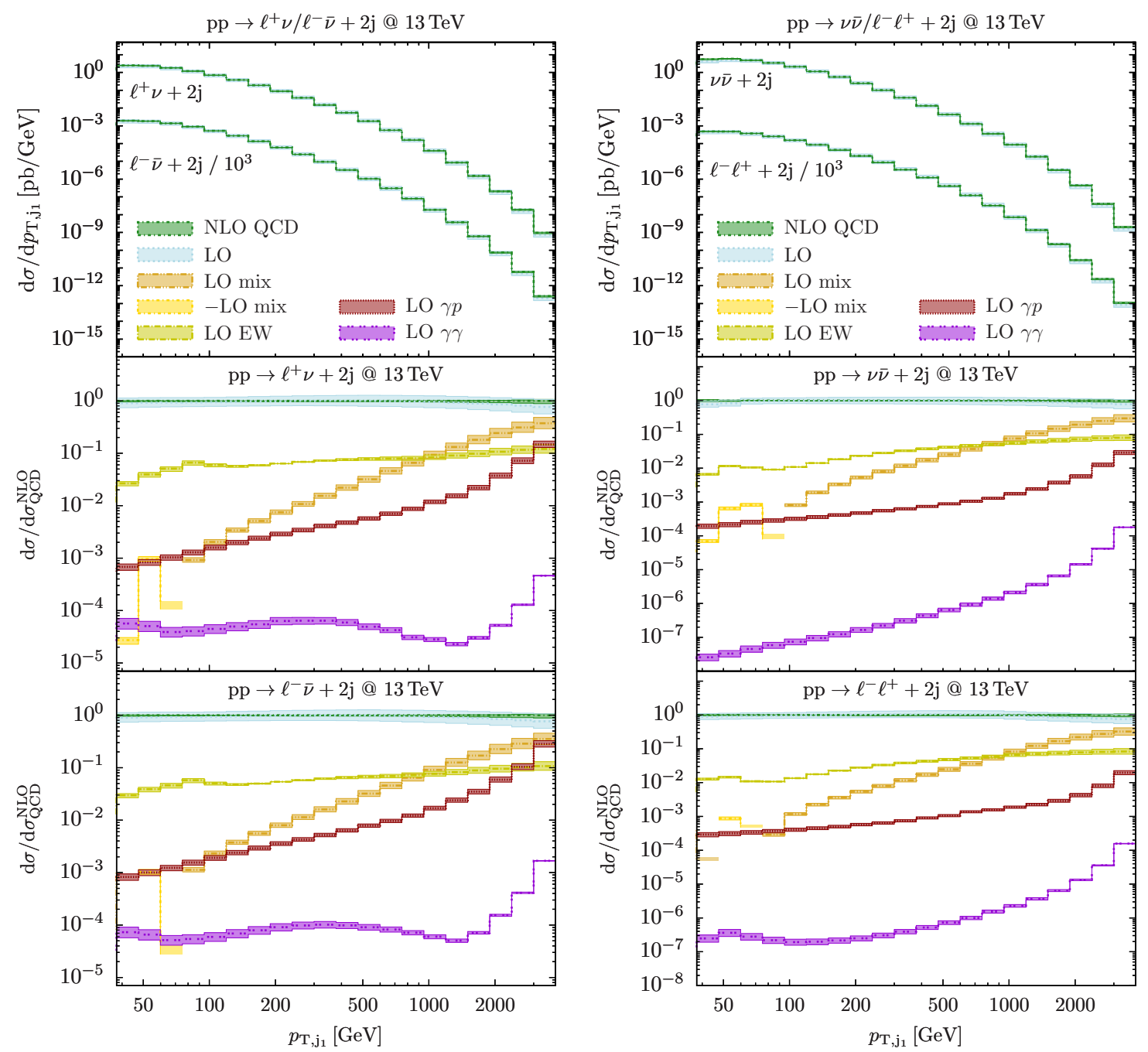

Figure 13. Distributions in the transverse momenta of the hardest jet $p_{\mathrm{T}, j_{1}}$ for off-shell $\mathrm{W}^{ \pm}+2 j$ (left) and $\mathrm{Z}+2 j$ (right) production and decay. Curves and bands as in figure 12 .

production. In this section, using a naïve merging approach based on exclusive sums [93] we illustrate how the combination of one- and two-jet NLO samples can stabilise the perturbative QCD convergence of one-jet inclusive observables and guarantee a consistent behaviour of EW corrections. Exclusive sums consist of combinations of fixed-order NLO calculations with variable jet multiplicity, where double counting is avoided by imposing appropriate cuts on the jet transverse momenta [93]. To combine $V+1$ jet and $V+2$ jet samples, we use the dimensionless variable

$$
r_{2 / 1}=\frac{p_{\mathrm{T}, j_{2}}}{p_{\mathrm{T}, j_{1}}}
$$

where $p_{\mathrm{T}, j_{1}}$ and $p_{\mathrm{T}, j_{2}}$ are the transverse momenta of the first two jets in the acceptance region (2.4), and $p_{\mathrm{T}, j_{2}}=0$ if there is only one jet within the acceptance. The exclusive sum is built by imposing a $r_{2 / 1}$ cut that separates the phase space into complementary regions, $r_{2 / 1}<r_{2 / 1}^{\text {cut }}$ and $r_{2 / 1}>r_{2 / 1}^{\text {cut }}$. In order to avoid a double counting of topologies with two 

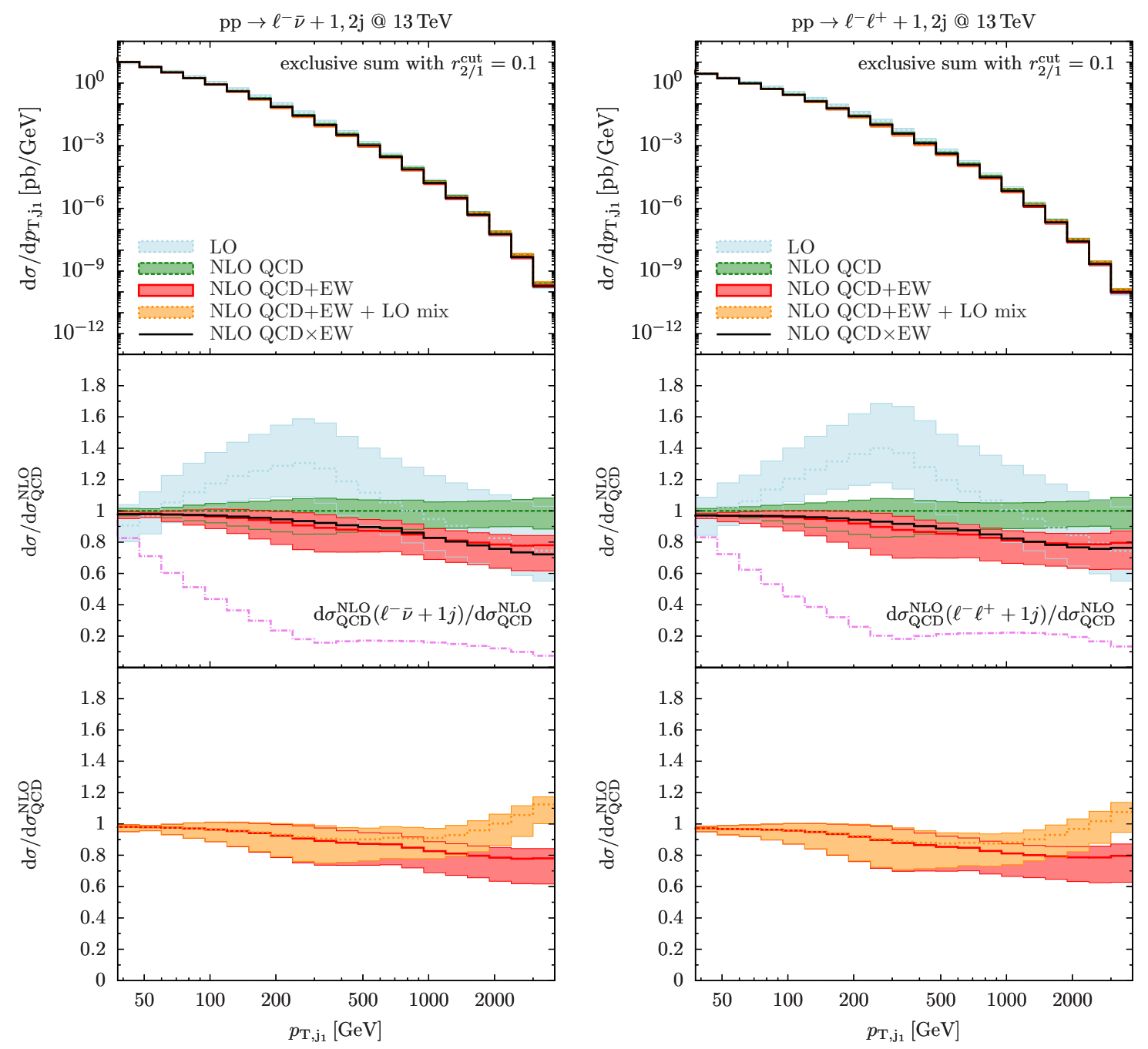

Figure 14. Differential distribution in the transverse momentum of the hardest jet, $p_{\mathrm{T}, j_{1}}$, for $\ell^{-} \bar{\nu}_{\ell}+$ jets (left) and $\ell^{+} \ell^{-}+$jets (right). Shown are predictions merged with exclusive sums using $r_{2 / 1}^{\text {cut }}=0.1$. The upper panels display absolute LO (light blue), NLO QCD (green), NLO QCD+EW (red), $\mathrm{NLO} \mathrm{QCD} \times \mathrm{EW}$ (black) and $\mathrm{NLO} \mathrm{QCD}+\mathrm{EW}+\mathrm{LO}$ mix (orange) predictions, where "LO mix" denotes QCD-EW mixed Born contributions of $\mathcal{O}\left(\alpha_{\mathrm{S}} \alpha^{3}\right)$ in the two-jet sample. Relative corrections with respect to NLO QCD are displayed in the lower panels. The bands correspond to scale variations, and in the case of ratios only the numerator is varied. The dashed magenta curves illustrate the relative importance of one-jet contributions $\left(r_{2 / 1}<r_{2 / 1}^{\text {cut }}\right)$ with respect to the combined one- and two-jet sub-samples at NLO QCD .

hard jets, the $V+1$ jet sample is restricted to the region $r_{2 / 1}<r_{2 / 1}^{\text {cut }}$, which corresponds to one-jet topologies, whereas the $V+2$ jet sample is used to populate the $r_{2 / 1}>r_{2 / 1}^{\text {cut }}$ region, characterised by the presence of two hard jets.

In figures 14 and 15 we present leading-jet and vector-boson $p_{\mathrm{T}}$ distributions for inclusive $\ell^{-} \bar{\nu}_{\ell}+$ jets and $\ell^{+} \ell^{-}+$jets production, where the one- and two-jet contributions are combined using a separation cut $r_{2 / 1}=0.1$. In the $p_{\mathrm{T}}$ distribution of the hardest jet we observe, as expected, that above a few hundred $\mathrm{GeV}$ the impact of two-jet topologies is 

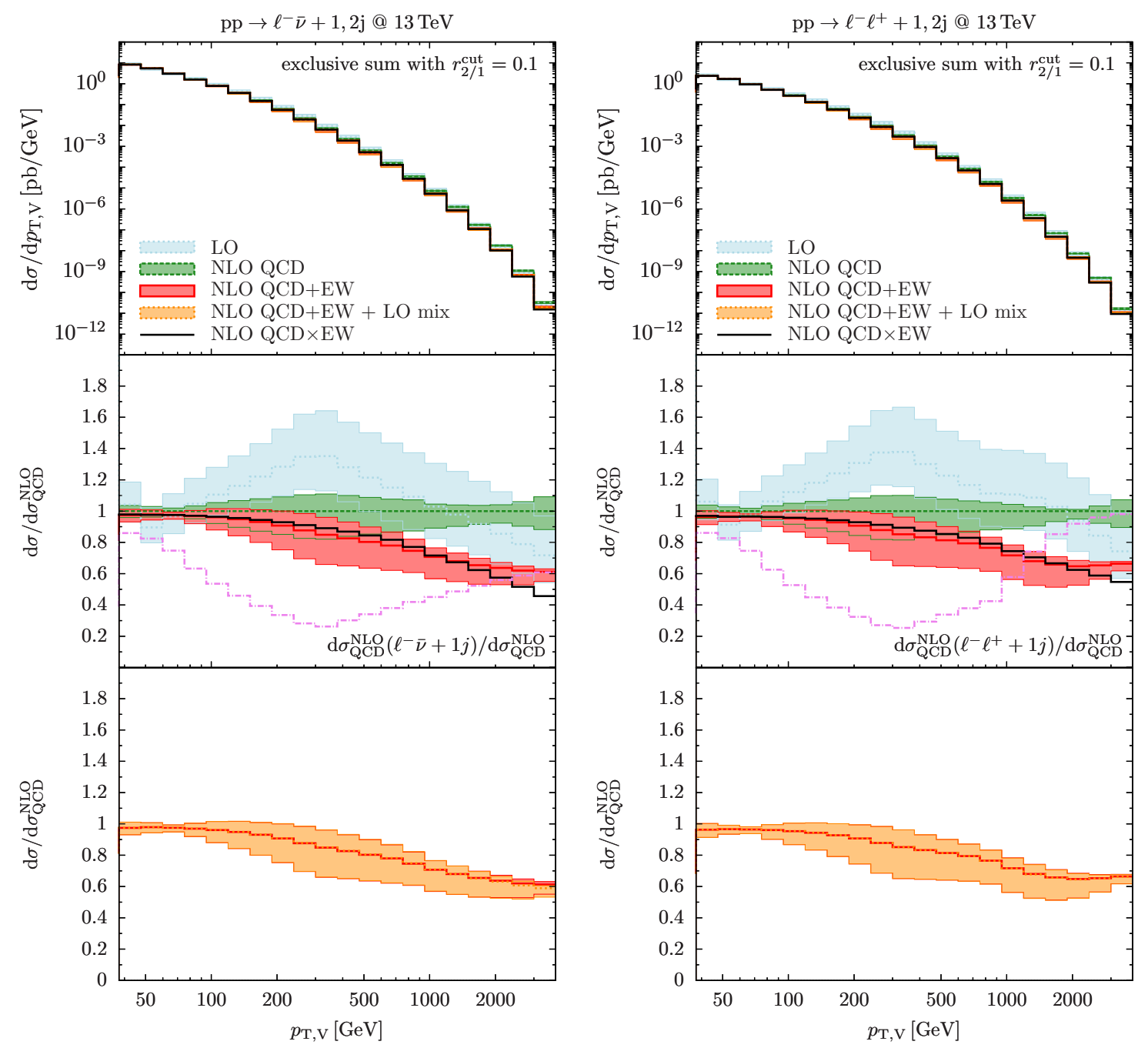

Figure 15. Differential distribution in the transverse momentum of the reconstructed vector boson, $p_{\mathrm{T}, \mathrm{V}}$, for $\ell^{-} \bar{\nu}_{\ell}+$ jets (left) and $\ell^{+} \ell^{-}+$jets (right). Shown are predictions merged with exclusive sums using $r_{2 / 1}^{\text {cut }}=0.1$. Curves and bands as in figure 14 .

overwhelming. In contrast, for $p_{\mathrm{T}, j_{1}}<300 \mathrm{GeV}$ their contribution tends to be suppressed by the the acceptance cut on the second jet, $p_{\mathrm{T}, j_{2}}>30 \mathrm{GeV}$, which effectively corresponds to $r_{2 / 1}^{\text {cut }}=30 \mathrm{GeV} / p_{\mathrm{T}, j_{1}}>0.1$. Thanks to the fact that the huge contributions from two-jet topologies are included starting from Born level and supplemented by NLO QCD+EW corrections, the exclusive-sums approach leads to a drastic improvement of the perturbative convergence as compared to fixed-order predictions for inclusive $V+$ jet production in figure 5 (left). In fact, in the full $p_{\mathrm{T}}$ range considered we observe moderate NLO QCD corrections and scale uncertainties. Moreover, NLO EW effects in figure 14 feature a consistent Sudakov behaviour, with $-20 \%$ corrections around $2 \mathrm{TeV}$. Including also QCD-EW mixed Born terms of $\mathcal{O}\left(\alpha_{\mathrm{S}} \alpha^{3}\right)$ (LO mix) in the two-jet sample, we observe that at the $\mathrm{TeV}$ scale their contribution becomes sizable and can even overcompensate the negative effects of EW Sudakov type. Apart from these quantitative considerations, it is important to 
realise that mixed Born contributions in the two-jet region $\left(r_{2 / 1}>r_{2 / 1}^{\text {cut }}\right)$ represent the natural continuation of NLO mixed bremsstrahlung in the one-jet region $\left(r_{2 / 1}>r_{2 / 1}^{\text {cut }}\right)$. Their inclusion is thus crucial for a consistent combination of different jet multiplicities.

In the vector-boson $p_{\mathrm{T}}$ distribution (figure 15 ) we observe that, similarly as in figure 14, the relative weight of $V+2$ jet topologies grows with $p_{\mathrm{T}}$ up to about $300 \mathrm{GeV}$ as a result of the acceptance cut on the second jet. However, in contrast to the case of the jet $p_{\mathrm{T}}$, in the region of high vector-boson $p_{\mathrm{T}}$, where the separation cut $r_{2 / 1}^{\text {cut }}=0.1$ comes into play, we see that one-jet contributions become increasingly important again. This indicates that the higher a boost of the $\mathrm{W}$ boson is required by the observable, the less likely it is to have two jets of comparable $p_{\mathrm{T}}$, leading to a hierarchical pattern of QCD radiation. In this situation NLO calculations for $V+1$ jet prodution are expected to be reliable, and in fact we find that inclusive $V+1$ jet predictions and exclusive sums provide similarly well behaved results. In both cases the quality of the perturbative QCD expansion turns out to be good, and in the multi-TeV regime we observe the usual negative NLO EW effects, which can become as large as $-40 \%$. We also note that, as compared to fixed-order $V+1$ jet inclusive results in figure 5 (left), exclusive sums lead to a smaller difference between the QCD+EW and $\mathrm{QCD} \times \mathrm{EW}$ prescriptions. Finally, at high vector-boson $p_{\mathrm{T}}$ we find that, consistent with the subleading role of two-jet topologies, mixed Born contributions to $V+2$ jets are irrelevant.

\subsection{Virtual approximation of NLO EW corrections}

As discussed in the following, virtual EW corrections with an appropriate infrared subtraction can provide a fairly accurate approximation of exact NLO EW effects. The fact that such an approximation does not require the explicit integration of subtracted real-emission matrix elements represents an important technical simplification. In particular, since Born and infrared-subtracted EW virtual contributions live on the same $n$-parton phase space, the combination of contributions with variable jet multiplicity can be realised with a multijet merging approach of LO complexity. The main physical motivation for a virtual EW approximation is given by the fact that Sudakov EW logarithms - the main source of large NLO EW effects at high energy - arise only from virtual corrections. Moreover, in various cases, such as for vector-boson production in association with one [33] or two jets [45], it turns out that a virtual EW approximation can provide percent-level accuracy for a wide range of observables and energy scales, also well beyond the kinematic regions where Sudakov EW logarithms become large.

Motivated by these observations, we adopt the following virtual approximation for the NLO EW corrections to $V+n$ jet production,

$$
\mathrm{d} \sigma_{n, \mathrm{NLOEW}}=\left[\mathrm{B}_{n}\left(\Phi_{n}\right)+\mathrm{V}_{n, \mathrm{EW}}\left(\Phi_{n}\right)+\mathrm{I}_{n, \mathrm{EW}}\left(\Phi_{n}\right)\right] \mathrm{d} \Phi_{n} .
$$

Here, $\mathrm{B}_{n}\left(\Phi_{n}\right)$ stands for the Born contribution of $\mathcal{O}\left(\alpha_{\mathrm{S}}^{n} \alpha^{2}\right)$, and $\mathrm{V}_{n, \mathrm{EW}}\left(\Phi_{n}\right)$ denotes the exact one-loop EW corrections of $\mathcal{O}\left(\alpha_{\mathrm{S}}^{n} \alpha^{3}\right)$. The cancellation of virtual infrared singularities is implemented through the $\mathrm{I}_{n, \mathrm{EW}}\left(\Phi_{n}\right)$ term, which represents the NLO EW generalisation of the Catani-Seymour I operator [41, 94, 95]. This latter term does not contain the EW $\mathbf{K}$ and $\mathbf{P}$ operators. It results from the endpoint term of the analytic integration over all 
dipole subtraction terms of $\mathcal{O}\left(\alpha_{\mathrm{S}}^{n} \alpha^{3}\right)$, which arise from the insertion of QED and QCD dipole kernels in $\mathcal{O}\left(\alpha_{\mathrm{S}}^{n} \alpha^{2}\right)$ squared Born matrix elements and $\mathcal{O}\left(\alpha_{\mathrm{S}}^{n-1} \alpha^{3}\right)$ QCD-EW mixed Born terms, respectively.

In the following the shorthand $\mathrm{EW}_{\text {virt }}$ will be used to denote the virtual EW approximation of (5.2). The accuracy of this approximation is illustrated in figures 16-19 by comparing it to exact NLO EW results for various (physical and unphysical) differential observables in $p p \rightarrow \ell \ell / \ell \nu / \nu \nu+1,2$ jet production. ${ }^{8}$ Exact and approximate results are compared both for the case of a conventional NLO calculation for $V+1$ jet $\left(r_{2 / 1}^{\text {cut }}=1\right)$ and combining NLO predictions for $V+1,2$ jets with exclusive sums $\left(r_{2 / 1}^{\text {cut }}=0.1\right)$. Exclusive sums provide a quantitative indication of the accuracy of the $\mathrm{EW}_{\text {virt }}$ approximation in a framework that mimics, although in a rough way, the multijet merging approach that will be adopted in sections 5.3-5.5.

For the various processes and distributions in figures 16-19 the $\mathrm{EW}_{\text {virt }}$ approximation turns out to be in generally good agreement with exact NLO predictions. The most striking exception is given by the $m_{\ell \ell}$ and $m_{\ell \nu}$ invariant-mass distributions in the off-shell region below the Breit-Wigner peak. In this case, real QED radiation off the charged leptons leads to corrections of a few tens of percent, which can not be reproduced by the $\mathrm{EW}_{\text {virt }}$ approximation as exclusive real photon emission is not included. In contrast, for distributions in the transverse momentum of the vector bosons or of the charged leptons that arise from their decays, we observe very good agreement, typically at the 1-2\% level, from low $p_{\mathrm{T}}$ up to the multi- $\mathrm{TeV}$ region.

The leading-jet $p_{\mathrm{T}}$ distribution represents a special case. Here, the $\mathrm{EW}_{\text {virt }}$ approximation performs quite well up to about $500 \mathrm{GeV}$, but at the $\mathrm{TeV}$ scale it is plagued by sizable inaccuracies. We have checked that this is largely due to the contribution of mixed bremsstrahlung, i.e. to the QCD-EW interference between matrix elements that describe the real emission of QCD partons at $\mathcal{O}\left(\alpha_{\mathrm{S}}^{n} \alpha^{3}\right)$. Such contributions are not covered by the $\mathrm{EW}_{\text {virt }}$ approximation, while in a standard NLO EW calculation for $V+1$ jet $\left(r_{2 / 1}^{\text {cut }}=1\right)$ they can reach $30-50 \%$ in the multi- $\mathrm{TeV}$ region. In contrast, in the exclusive-sums approach mixed bremsstrahlung is suppressed by the separation cut between 1-jet and 2-jet regions $\left(r_{2 / 1}^{\text {cut }}=0.1\right)$, and the discrepancy between exact EW corrections and $\mathrm{EW}_{\text {virt }}$ approximation is reduced to less than $10 \%$ at $3 \mathrm{TeV}$. On the one hand, this level of agreement can be further improved by lowering the value of the separation cut. Thus in our implementation of multijet merging we will adopt a merging cut that corresponds to $r_{2 / 1}^{\text {cut }} \ll 0.1$ in the multi$\mathrm{TeV}$ region. On the other hand, for a realistic description of EW effects, it is clear that the sizable contribution from mixed bremsstrahlung should be included also above the merging cut. In the MEPS framework described in section 5.4, this will be achieved by complementing any $n$-jet Born contribution of $\mathcal{O}\left(\alpha_{\mathrm{S}}^{n} \alpha^{2}\right)$ by mixed Born contributions of $\mathcal{O}\left(\alpha_{\mathrm{S}}^{n-1} \alpha^{3}\right)$ for any jet multiplicity $n \geq 2$ that is included in the merging procedure. ${ }^{9}$ Such mixed Born contributions will provide an effective description of mixed bremsstrahlung that arises from

\footnotetext{
${ }^{8}$ Process-dependent correction factors are introduced in figures 16-19 such that the integrated NLO $\mathrm{QCD}+\mathrm{EW}_{\text {virt }}$ predictions match the complete NLO QCD+EW results. These factors are $k_{\nu_{\ell} \bar{\nu}_{\ell}} \approx 1.00$ for $\nu_{\ell} \bar{\nu}_{\ell}+$ jets, $k_{\ell \nu} \approx 0.99$ for $\ell \nu+$ jets and $k_{\ell \ell} \approx 0.98$ for $\ell^{+} \ell^{-}+$jets.

${ }^{9}$ Note that mixed Born contributions do not exist for $n \leq 1$.
} 

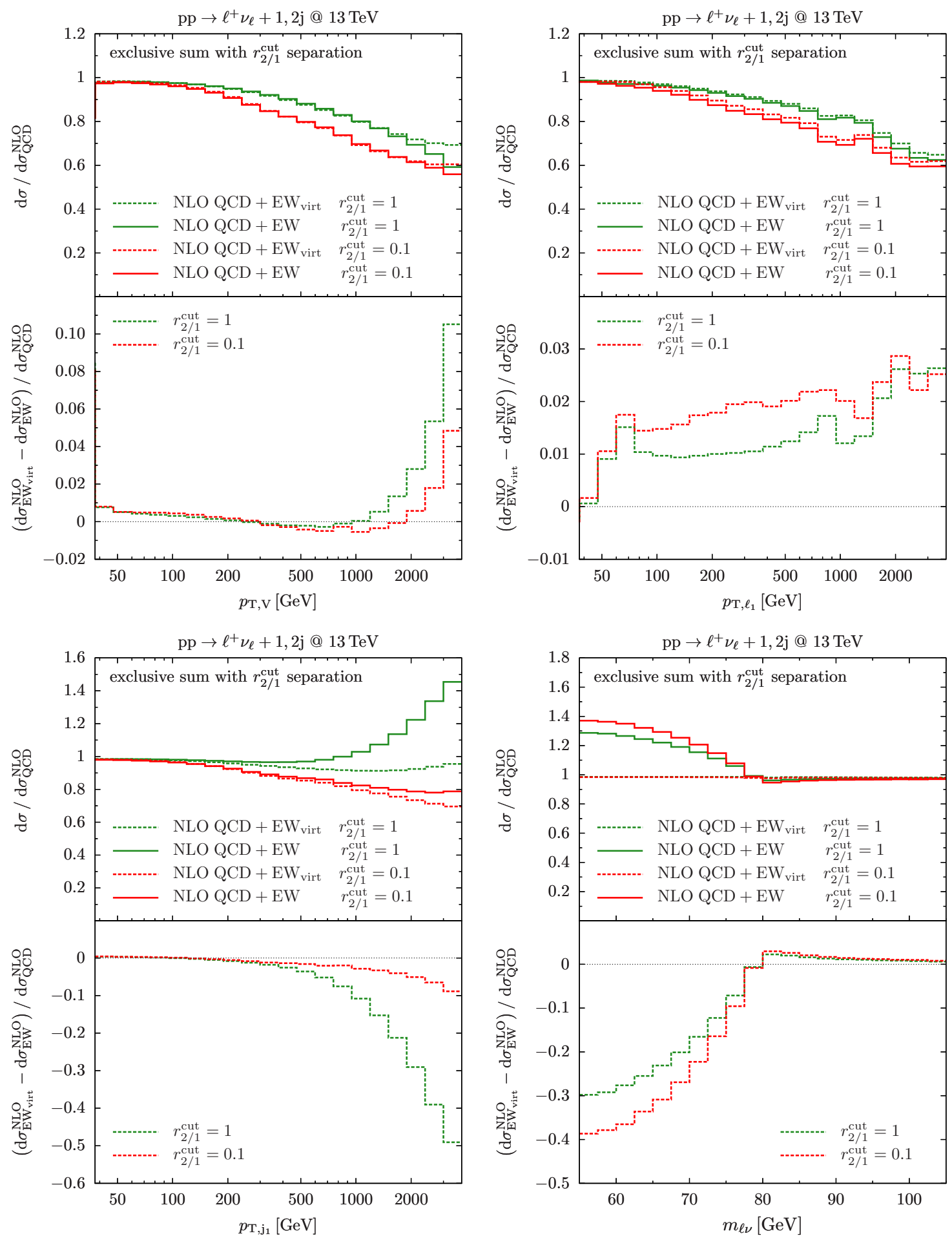

Figure 16. Exact NLO EW predictions for $\ell^{+} \nu_{\ell}+$ jets production at $13 \mathrm{TeV}$ are compared to the virtual approximation (NLO EW $\mathrm{E}_{\text {virt }}$ ) of (5.2). All results are normalised to NLO QCD predictions. The red curve represent parton-level predictions for $\ell^{+} \nu_{\ell}+1,2$ jet combined in the exclusive-sums approach with a separation cut $r_{2 / 1}^{\text {cut }}=0.1$, while conventional predictions for $\ell^{+} \nu_{\ell}+1$ jet $\left(r_{2 / 1}^{\text {cut }}=1\right)$ are shown in green. 

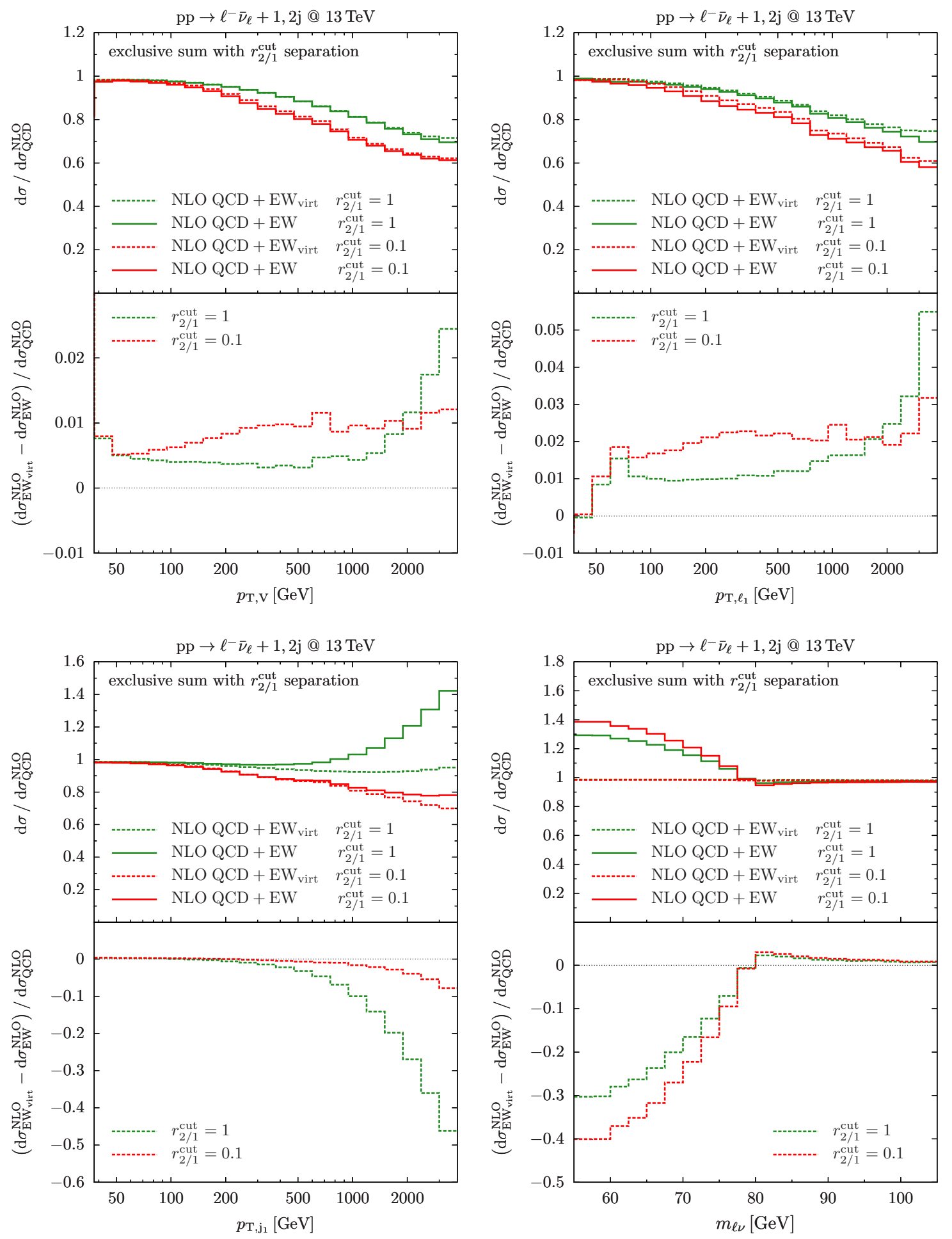

Figure 17. Exact NLO EW predictions for $\ell^{-} \bar{\nu}_{\ell}+$ jets production at $13 \mathrm{TeV}$ are compared to the

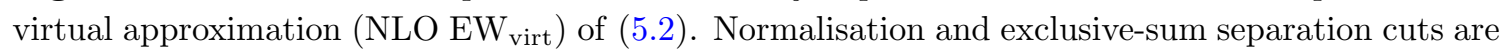
as in figure 16. 

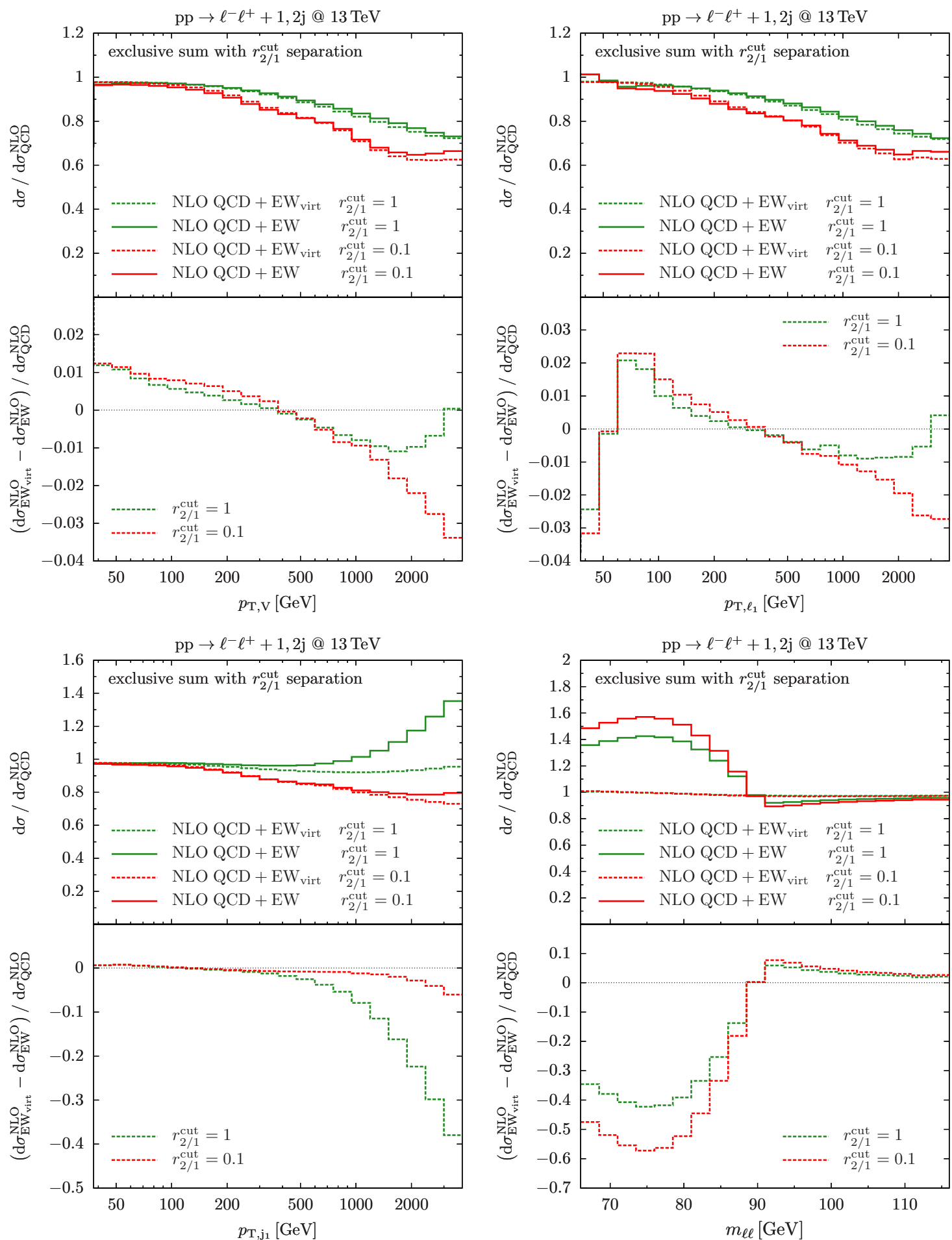

Figure 18. Exact NLO EW predictions for $\ell^{+} \ell^{-}+$jets production at $13 \mathrm{TeV}$ are compared to the virtual approximation (NLO EW $\mathrm{EW}_{\text {virt }}$ ) of (5.2). Normalisation and exclusive-sum separation cuts are as in figure 16 . 

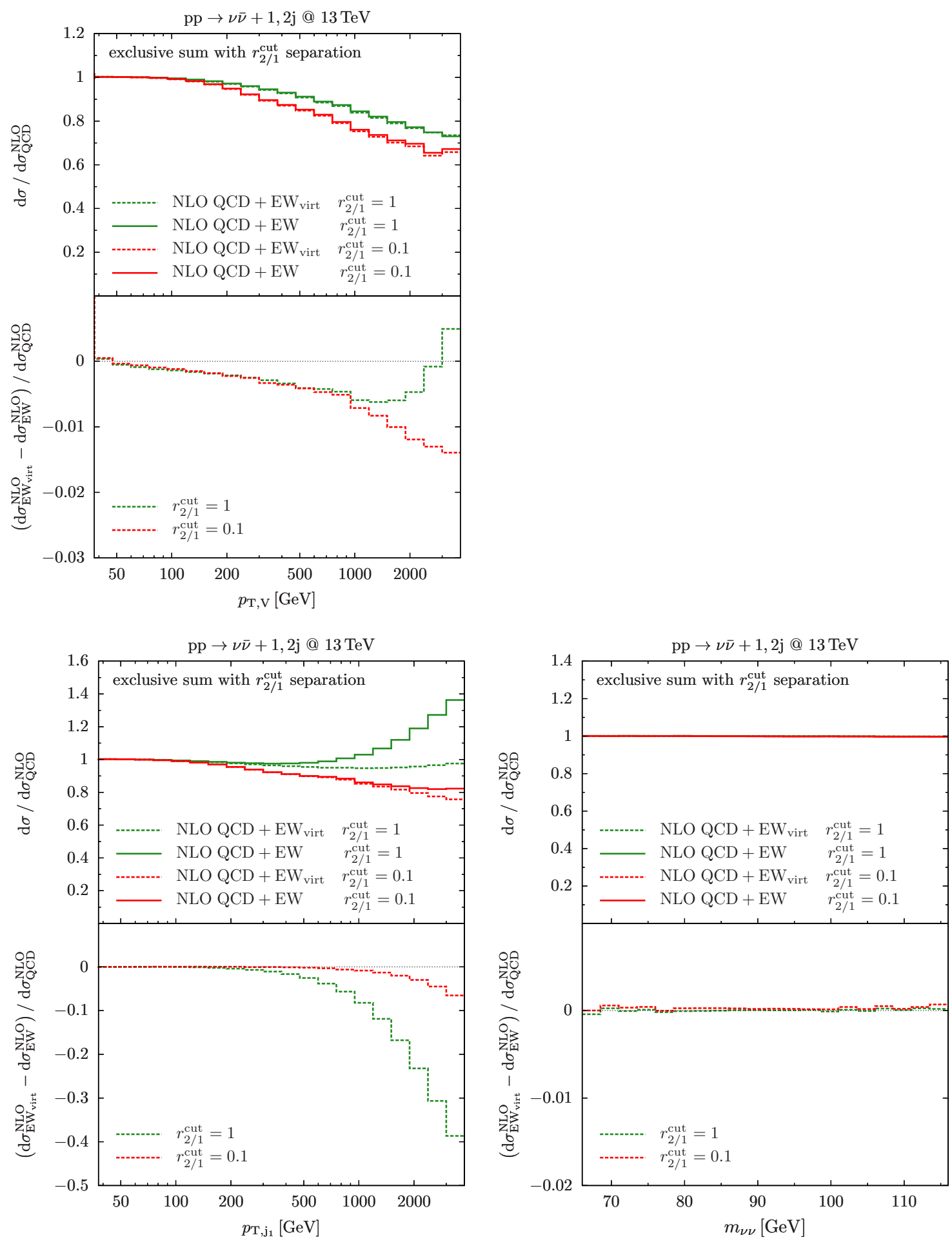

Figure 19. Exact NLO EW predictions for $\nu_{\ell} \bar{\nu}_{\ell}+$ jets production at $13 \mathrm{TeV}$ are compared to the virtual approximation $\left(\mathrm{NLO} \mathrm{EW}_{\text {virt }}\right)$ of (5.2). Normalisation and exclusive-sum separation cuts are as in figure 16. 
regions with $n-1$ jets. Moreover, their accuracy will be (approximately) increased by one order in $\alpha_{\mathrm{S}}$ through the implementation of NLO EW virt corrections of $\mathcal{O}\left(\alpha_{\mathrm{S}}^{n} \alpha^{3}\right)$.

In summary, in absence of kinematic constraints that confine vector bosons in the offshell regime below the resonance region, combining the $\mathrm{EW}_{\text {virt }}$ approximation of (5.2) with mixed bremsstrahlung contributions can reproduce NLO EW predictions with an accuracy of $1-2 \%$ up to transverse momenta of the order of $1 \mathrm{TeV}$ or more.

\subsection{MEPs merging at NLO QCD}

As a basis to combine NLO EW corrections with multijet merging, in this section we recapitulate the essential features of the MEPS merging method [54, 55, 92, 96]. This technique allows one to generate inclusive event samples with variable jet multiplicity in such a way that events with $n=0,1, \ldots, n_{\max }$ jets are described in terms of corresponding $n$-jet matrix elements at LO or NLO accuracy. To this end, resolved jets are separated from unresolved emissions by means of a so-called merging scale, $Q_{\text {cut }}$, and the phase space is split into different regions according to the number of resolved jets. More precisely, the phase-space partitioning is formulated in terms of the $k_{\mathrm{T}}$-type jet-resolution parameters $Q_{1}>Q_{2}>\cdots>Q_{n_{\max }}$, which represent the resolution scales of the first, second, and subsequent emissions. The $n$-jet regions for $0 \leq n<n_{\max }$ are thus defined through

$$
Q_{1}>\cdots>Q_{n}>Q_{\text {cut }}>Q_{n+1}>\cdots
$$

In the leading-order formulation of the MEPs method [92], called MEPs@Lo, the exclusive cross sections with $0 \leq n<n_{\max }$ resolved jets are generated according to ${ }^{10}$

$$
\mathrm{d} \sigma_{n}^{(\mathrm{MEPS} @ \mathrm{LO})}=\mathrm{d} \Phi_{n} \mathrm{~B}_{n}\left(\Phi_{n}\right) \Theta\left(Q_{n}-Q_{\text {cut }}\right) \mathcal{F}_{n}\left(\mu_{Q}^{2} ;<Q_{\text {cut }}\right),
$$

where $\mathrm{B}_{n}\left(\Phi_{n}\right)$ summarises the relevant squared Born matrix elements convoluted with PDFs and summed/averaged over all partonic degrees of freedom. The theta function ensures that all partons in the matrix elements correspond to resolved jets, while $\mathcal{F}_{n}\left(\mu_{Q}^{2} ;<\right.$ $\left.Q_{\text {cut }}\right)$ denotes a truncated vetoed parton shower that is restricted to the unresolved regions, $Q<Q_{\text {cut }}$, as explained in more detail below. For the highest matrix-element multiplicity, $n=n_{\max }$, the region $Q_{n_{\max }}>Q>Q_{\text {cut }}$ is inclusive with respect to higher-order radiation. Thus, the $Q_{\text {cut }}$-veto is relaxed to a $Q_{n_{\max }}$-veto, and the truncated parton shower can fill the whole phase space below.

The truncated vetoed shower supplements multijet matrix elements with Sudakov suppression factors that render resolved jet emissions equally exclusive as shower emissions. In combination with the CKKW scale choice [98, 99], this guarantees a smooth transition from matrix-element to parton-shower predictions across $Q_{\text {cut }}$ and ensures the restauration of the parton shower's resummation properties in the matrix-element region. As a result, the $Q_{\text {cut }}$ dependence of physical observables is kept at a formally subleading level with respect to the logarithmic accuracy of the parton shower. The implementation of the above aspects of the merging procedure requires, for each multijet event, the determination of

\footnotetext{
${ }^{10}$ Here we employ the notation of [97] in a slightly simplified form. For a more detailed discussion of technical aspects we refer to the original publications [54, 55, 92, 96].
} 
a would-be shower history consisting of a $2 \rightarrow 2$ core process, characterised by a certain core scale $\mu_{\text {core }}$, and a series of subsequent branchings at scales $t_{1}, t_{2}, \ldots, t_{M}$. In the MEPS approach, shower histories are determined by probabilistic clustering of multijet final states based on the inversion of the SHERPA parton shower.

The truncated parton shower $\mathcal{F}_{n}\left(\mu_{Q}^{2} ;<Q_{\text {cut }}\right)$ in (5.4) starts at the resummation scale $\mu_{Q}^{2}=t_{0}=\mu_{\text {core }}^{2}$ and is stopped and restarted at each reconstructed branching scale $t_{1}, \ldots, t_{M}$. At each stage a kernel corresponding to the actual partially clustered configuration is used. Finally, the shower terminates at the infrared cutoff, $t_{c}$. The Sudakov form factor that guarantees the exclusiveness of $n$-jet contributions is generated by vetoing the entire event in case of any resolved emission $\left(Q>Q_{\text {cut }}\right)$ of the truncated shower for $t_{0}>t>t_{c}$. Since the role of the Sudakov suppression is to avoid double counting between contributions with different numbers of resolved jets, unresolved emissions $\left(Q<Q_{\text {cut }}\right)$ are not vetoed. ${ }^{11}$

The factorisation scale is set equal to the core scale, $\mu_{F}=\mu_{\text {core, }}$ while the strong coupling $\alpha_{S}$ in multijet Born matrix elements is evaluated at the renormalisation scale $\mu_{R}=\mu_{\mathrm{CKKW}}$, defined through

$$
\alpha_{S}^{N}\left(\mu_{\text {CKKW }}^{2}\right)=\alpha_{S}^{N-M}\left(\mu_{\text {core }}^{2}\right) \alpha_{S}\left(t_{1}\right) \ldots \alpha_{S}\left(t_{M}\right),
$$

where $\alpha_{S}^{N}$ and $\alpha_{\mathrm{S}}^{N-M}$ are the overall $\alpha_{S}$ factors for the LO cross section of the actual multijet process and for the related $2 \rightarrow 2$ core process, respectively.

In the case of $V+$ jets, the shower history is determined by stepwise clustering of $V+$ multijet events based on the relative probability of all possible QCD and EW splitting processes, using matrix-element information to select allowed states only. ${ }^{12}$ In particular, also the creation of vector bosons and their (off-shell) decays are treated as possible splitting processes. Thus the clustering of $V+$ multijet events terminates with three possible $2 \rightarrow 2$ core processes: $p p \rightarrow 2 \ell, p p \rightarrow V j$ and $p p \rightarrow j j$. The corresponding default core scales in SHERPA $\operatorname{read}^{13}$

$$
\mu_{\mathrm{core}, \ell \ell}=m_{\ell \ell}, \quad \mu_{\mathrm{core}, V j}=\frac{1}{2} E_{T, V}=\frac{1}{2} \sqrt{M_{V}^{2}+p_{T, V}^{2}}, \quad \mu_{\mathrm{core}, j j}=\frac{1}{2}\left(\frac{1}{\hat{s}}-\frac{1}{\hat{t}}-\frac{1}{\hat{u}}\right)^{-\frac{1}{2}} .
$$

Note that excluding EW splittings from the clustering procedure would always lead to a Drell-Yan core process and a core scale $\mu_{\text {core }}=m_{\ell \ell}=\mathcal{O}\left(M_{\mathrm{Z}, \mathrm{W}}\right)$, which is clearly inappropriate at high transverse momenta. Including all QCD and EW splittings in the clustering algorithm is thus crucial for the consistent determination of the hard core processes and

\footnotetext{
${ }^{11}$ Note that, for $n$-jet configurations, in spite of $Q_{n}>Q_{\text {cut }}$, also truncated shower emissions with $t>t_{n}$ can give rise to unresolved jets with $Q<Q_{\text {cut }}$ due to the different nature of the shower evolution variable $t$ and the $k_{\mathrm{T}}$-measure $Q$.

${ }^{12}$ For example, in a $g q \rightarrow \ell^{+} \ell^{-} q$ configuration identifying a $q \rightarrow q g$ splitting would be allowed by the parton shower and preferred in many regions of phase space over the alternatives. However, this would lead to a $g g \rightarrow \ell^{+} \ell^{-}$configuration and, thus, identifying such a splitting needs to be prevented.

${ }^{13}$ The core scale $\mu_{\mathrm{core}, j j}$ is driven by the smallest Mandelstam invariant, i.e. by the scale associated with the dominant topology in the $p p \rightarrow j j$ core process. In practice $\mu_{\mathrm{core}, j j}$ is fairly close to the jet transverse momentum after clustering.
} 
the related scale. In particular, it allows for shower histories where $V+$ multijet production proceeds via hard dijet production and subsequent soft vector-boson emission, which corresponds to the dominant mechanism of $V+$ jets production at high jet $p_{\mathrm{T}}$.

The MEPs@NLO merging method [54,55] upgrades LO merging to NLO QCD in the MC@NLO framework [100-103]. It can be summarised through the following formula for exclusive $n$-jet cross sections,

$$
\begin{aligned}
\mathrm{d} \sigma_{n}^{(\mathrm{MePs} @ \mathrm{NLO})}= & {\left[\mathrm{d} \Phi_{n} \tilde{\mathrm{B}}_{n}\left(\Phi_{n}\right) \overline{\mathcal{F}}_{n}\left(\mu_{Q}^{2} ;<Q_{\text {cut }}\right)\right.} \\
& \left.+\mathrm{d} \Phi_{n+1} \tilde{\mathrm{H}}_{n}\left(\Phi_{n+1}\right) \Theta\left(Q_{\text {cut }}-Q_{n+1}\right) \mathcal{F}_{n+1}\left(\mu_{Q}^{2} ;<Q_{\text {cut }}\right)\right] \Theta\left(Q_{n}-Q_{\text {cut }}\right) .
\end{aligned}
$$

As discussed in more detail below, the $\tilde{\mathrm{B}}_{n}\left(\Phi_{n}\right)$ term corresponds to so-called soft events in MC@NLO and describes $n$ resolved partons $\left(Q_{n}>Q_{\text {cut }}\right)$ at matrix-element level including virtual corrections. The $\tilde{\mathrm{H}}_{n}\left(\Phi_{n+1}\right)$ term corresponds to so-called hard events in MC@NLO . It involves subtracted matrix elements with $n$ resolved partons, plus an additional parton whose emission is constrained in the unresolved region $\left(Q_{n+1}<Q_{\text {cut }}\right)$ in order to avoid double counting with matrix elements of higher jet multiplicity. Of course, for $n=n_{\max }$ this constraint on the real emission is not required, and the corresponding theta function in (5.7) is omitted.

Similarly as in the LO case, soft and hard events in (5.7) are used as seeds of truncated vetoed parton showers with starting scale $\mu_{Q}=\mu_{\text {core }}$ and a veto against emissions with $Q>Q_{\text {cut }}$. The veto is relaxed when the maximum jet multiplicity $n=n_{\max }$ is reached. In MePs@NLO, the truncated shower that is applied to soft events, $\overline{\mathcal{F}}_{n}\left(\mu_{Q}^{2} ;<Q_{\text {cut }}\right)$, is matched to the first matrix-element emission. To this end, the first emission is generated by the kernel ${ }^{14}[96,104]$

$$
\begin{aligned}
\tilde{\mathrm{D}}_{n}\left(\Phi_{n+1}\right)= & \mathrm{D}_{n}\left(\Phi_{n+1}\right) \Theta\left(t_{n}-t_{n+1}\right) \\
& +\left.\sum_{j=0}^{n-1} \mathrm{~B}_{n}\left(\Phi_{n}\right) \mathrm{K}_{j}\left(\Phi_{1, n+1}\right) \Theta\left(t_{j}-t_{n+1}\right) \Theta\left(t_{n+1}-t_{j+1}\right)\right|_{t_{0}=\mu_{Q}^{2}} .
\end{aligned}
$$

Here, $\mathrm{D}_{n}\left(\Phi_{n+1}\right)$ denotes exact Catani-Seymour subtraction terms. They are used to generate emissions with hardness $t_{n+1}<t_{n}$, which arise from $n$-parton configurations, and they match the full-colour infrared singularity structure of real-emission matrix elements. The remaining terms in (5.8) describe intermediate emissions with hardness $t_{n+1} \in\left[t_{j}, t_{j+1}\right]$ that arise from partially clustered configurations with $0 \leq j<n$ partons and corresponding Catani-Seymour kernels $\mathrm{K}_{j}$ in the usual leading-colour approximation of the parton shower. The matching of the truncated vetoed parton shower to the first NLO emission results in the following expression for hard events,

$$
\tilde{\mathrm{H}}_{n}\left(\Phi_{n+1}\right)=\mathrm{R}_{n}\left(\Phi_{n+1}\right)-\tilde{\mathrm{D}}_{n}\left(\Phi_{n+1}\right) \Theta\left(\mu_{Q}^{2}-t_{n+1}\right)
$$

\footnotetext{
${ }^{14}$ Here the veto against emissions with $Q>Q_{\text {cut }}$ is implicitly understood.
} 
where $\mathrm{R}_{n}\left(\Phi_{n+1}\right)$ stands for real-emission matrix elements. The soft term in (5.7) reads

$$
\tilde{\mathrm{B}}_{n}\left(\Phi_{n}\right)=\mathrm{B}_{n}\left(\Phi_{n}\right)+\tilde{\mathrm{V}}_{n}\left(\Phi_{n}\right)+\int \mathrm{d} \Phi_{1} \tilde{\mathrm{D}}_{n}\left(\Phi_{n}, \Phi_{1}\right) \Theta\left(\mu_{Q}^{2}-t_{n+1}\right) .
$$

It comprises a Born contribution, $\mathrm{B}_{n}\left(\Phi_{n}\right)$, a term $\tilde{\mathrm{V}}_{n}\left(\Phi_{n}\right)$ consisting of virtual QCD corrections and initial-state collinear counterterms, ${ }^{15}$ and the integrated subtraction terms (5.8) associated with the truncated parton shower. Similarly as for LO merging, we set $\mu_{F}=\mu_{\text {core }}$, and the renormalisation scale is chosen according to (5.5).

\subsection{Extension of MEPs merging to NLO QCD+EW}

Let us now turn to the extension of the MEPs@NLO formalism to also include NLO EW effects. While the method that we are going to introduce is entirely general, for the convenience of the discussion, in the following we will adopt a counting of $\alpha_{\mathrm{S}}$ and $\alpha$ couplings that corresponds to the specific case of $V+$ multijet production with off-shell vector-boson decays. In this case, in phase-space regions with $n$ resolved jets, LO and NLO QCD contributions of $\mathcal{O}\left(\alpha_{\mathrm{S}}^{n} \alpha^{2}\right)$ and $\mathcal{O}\left(\alpha_{\mathrm{S}}^{n+1} \alpha^{2}\right)$ will be supplemented by NLO EW corrections of $\mathcal{O}\left(\alpha_{\mathrm{S}}^{n} \alpha^{3}\right)$ and mixed QCD-EW Born terms of $\mathcal{O}\left(\alpha_{\mathrm{S}}^{n-1} \alpha^{3}\right)$.

Besides all relevant tree plus virtual amplitudes and Catani-Seymour counterterms which are already available in SHERPA+OPENLOOPS in the framework of fixed-order NLO QCD+EW automation - a complete implementation of MEPs merging at NLO QCD+EW requires additional technical ingredients that are still missing to date. In particular, the SHERPA parton shower, extended to QCD+QED, should be matched to the real emission of photons and QCD partons at $\mathcal{O}\left(\alpha_{\mathrm{S}}^{n} \alpha^{3}\right)$ in the S-MC@NLO framework. Moreover, a consistent showering and clustering approach for events associated with mixed QCD-EW matrix elements is needed. While we expect that such technical prerequisites will be fulfilled in the near future, based on the good quality of the NLO EW virt approximation of section 5.2 and the fact that it does not require resolved emissions of photons or QCD partons at NLO EW, in the following we present a first approximate, but reliable, extension of NLO multijet merging to also include NLO EW effects. This approach is based on the implementation of the NLO EW virt approximation in the $\tilde{\mathrm{B}}_{n}\left(\Phi_{n}\right)$ soft term of (5.7). While all other aspects of MEPS@NLO, including the truncated vetoed QCD parton shower, are kept unchanged, the NLO EW improved $n$-jet soft term takes the form

$$
\tilde{\mathrm{B}}_{n, \mathrm{QCD}+\mathrm{EW}}\left(\Phi_{n}\right)=\tilde{\mathrm{B}}_{n}\left(\Phi_{n}\right)+\mathrm{V}_{n, \mathrm{EW}}\left(\Phi_{n}\right)+\mathrm{I}_{n, \mathrm{EW}}\left(\Phi_{n}\right)+\mathrm{B}_{n, \text { mix }}\left(\Phi_{n}\right) .
$$

Here $\tilde{\mathrm{B}}_{n}\left(\Phi_{n}\right)$ is the usual NLO QCD soft term (5.11), and $\mathrm{B}_{n, \text { mix }}\left(\Phi_{n}\right)$ denotes QCD-EW mixed Born contributions of $\mathcal{O}\left(\alpha_{\mathrm{S}}^{n-1} \alpha^{3}\right)$. The terms $\mathrm{V}_{n, \mathrm{EW}}\left(\Phi_{n}\right)$ and $\mathrm{I}_{n, \mathrm{EW}}\left(\Phi_{n}\right)$ represent the renormalised virtual corrections of $\mathcal{O}\left(\alpha_{\mathrm{S}}^{n} \alpha^{3}\right)$ and the NLO EW generalisation of the Catani-Seymour I operator, respectively, as discussed in section 5.2.

The $\mathrm{I}_{n, \mathrm{EW}}$ term cancels all $\mathcal{O}\left(\alpha_{\mathrm{S}}^{n} \alpha^{3}\right)$ infrared divergences in the virtual EW corrections. This corresponds to an approximate and fully inclusive description of the emission

\footnotetext{
${ }^{15}$ Such contributions correspond to the $\mu_{F}$ dependent part of the integrated $\mathbf{P}$ operator in the CataniSeymour approach.
} 
of photons and QCD partons at $\mathcal{O}\left(\alpha_{\mathrm{S}}^{n} \alpha^{3}\right)$. More precisely, only contributions of soft and final-state-collinear type are included, while initial-state collinear contributions and related PDF counterterms ( $\mathbf{K}$ and $\mathbf{P}$ operators in the Catani-Seymour framework) are not taken into account. This implies a (small) spurious $\mathcal{O}\left(\alpha_{\mathrm{S}}^{n} \alpha^{3}\right)$ dependence associated to the uncancelled factorisation scale dependence of the $\mathcal{O}\left(\alpha_{\mathrm{S}}^{n} \alpha^{2}\right)$ and $\mathcal{O}\left(\alpha_{\mathrm{S}}^{n-1} \alpha^{3}\right)$ Born terms. In contrast, all relevant ultraviolet divergences and related renormalisation scale variations of $\mathcal{O}\left(\alpha_{\mathrm{S}}^{n} \alpha^{3}\right)$ are consistently included and cancelled. To this end, virtual EW corrections $\left(\mathrm{V}_{n, \mathrm{EW}}\right)$ and QCD-EW mixed Born terms $\left(\mathrm{B}_{n, \mathrm{mix}}\right)$ have to be kept together in (5.12), since only their combination is free from renormalisation-scale logarithms at $\mathcal{O}\left(\alpha_{\mathrm{S}}^{n} \alpha^{3}\right)$. This approach will be denoted as MEPs@NLO QCD+EW virt in the following.

Concerning the accuracy of the approximation (5.12) a few comments are in order. First of all, thanks to the exact treatment of virtual EW corrections, all possible large virtual EW effects related to Sudakov logarithms are included by construction. Moreover, the merging approach guarantees that EW correction effects are consistently included also in phase-space regions of higher jet multiplicity. Secondly, as pointed out in section 5.2, sizable NLO EW contributions can arise also from the emission of QCD partons through mixed QCD-EW matrix elements at NLO. As far as equation (5.12) is concerned, such mixed bremsstrahlung contributions are only included in a fully inclusive and approximate way through the $\mathrm{I}_{n \text {,EW }}$ operator. Nevertheless, the fact that mixed Born terms $\left(\mathrm{B}_{n, \mathrm{mix}}\right)$ are effectively merged at LO guarantees a fairly reliable and fully exclusive description of mixed bremsstrahlung also at high jet transverse momenta, where the effects can be sizable. Technically, unresolved $\left(Q_{n+1}<Q_{\text {cut }}\right)$ mixed bremsstrahlung of $\mathcal{O}\left(\alpha_{\mathrm{S}}^{n} \alpha^{3}\right)$ is generated by the interplay of the $\mathcal{O}\left(\alpha_{\mathrm{S}}^{n-1} \alpha^{3}\right) \mathrm{B}_{n \text {,mix }}$ terms with the QCD parton shower, and its resolved counterpart $\left(Q_{n+1}>Q_{\text {cut }}\right)$ is described by the Born mixed matrix elements with one extra jet, $\mathrm{B}_{n+1, \text { mix }}$. Finally, let us note that genuine QED bremsstrahlung at $\mathcal{O}\left(\alpha_{\mathrm{S}}^{n} \alpha^{3}\right)$ is only included through the naïve and inclusive approximation provided by the $\mathrm{I}_{n, \mathrm{EW}}$ term. Thus, the approximation (5.12) cannot account for large QED logarithms that can appear in differential distributions for bare leptons and similar exclusive observables. Nevertheless, for a wide range of physical observables the impact of QED bremsstrahlung tends to be negligible. This is the case also for many leptonic observables if photon bremsstrahlung is treated in a rather inclusive way, e.g. through the recombination of collinear photon emissions. In any case, leading-logarithmic QED effects could be easily included in (5.12) by a simple QCD+QED extension of the parton shower [105] or a YFS-type soft photon resummation [106], without having to match the QED part to NLO QCD+QED matrix elements. This pure shower approach could be further improved by including photonemission matrix elements via LO merging [105], in a similar way as discussed above for the case of mixed bremsstrahlung.

The NLO QCD+EW extension of the MEPS@NLO method based on equation (5.12) was implemented in SHERPA+OpenLoops in a fully automated way and applied to $V+$ multijet production as described in the following section.

\subsection{Numerical MEPs@NLO QCD+EW results for $p p \rightarrow V+0,1,2$ jets}

Based on the above described multijet merging method, in this section we present an inclusive simulation of $\ell^{-} \bar{\nu}_{\ell}+$ multijet production that includes NLO QCD $+\mathrm{EW}_{\text {virt }}$ correction 

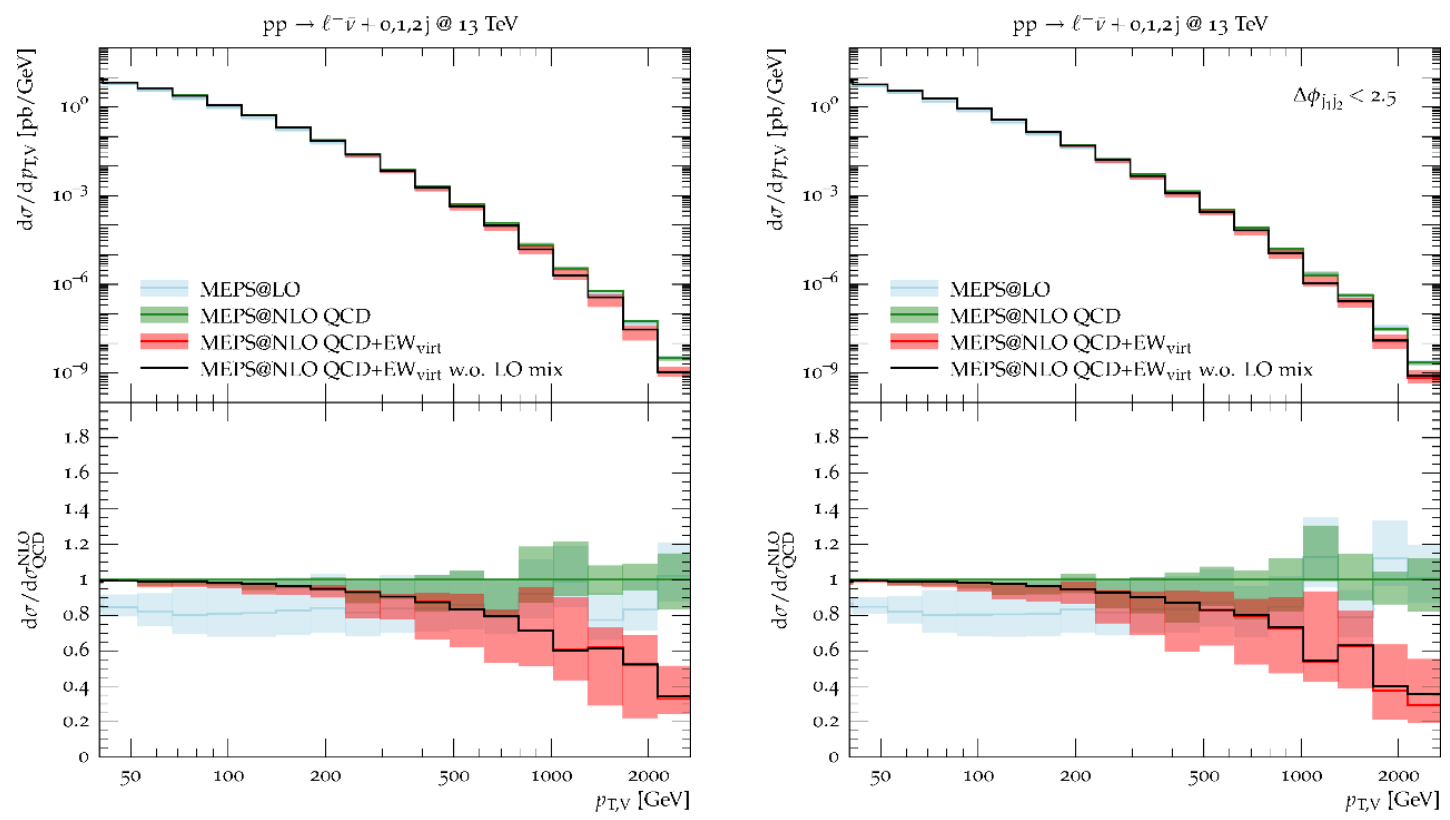

Figure 20. Distribution in the transverse momentum of the reconstructed vector boson in $\ell^{-} \bar{\nu}_{\ell}+$ jets production with standard cuts (left) and in presence of an extra cut $\Delta \phi_{j_{1} j_{2}}<2.5$ (right). The upper frame displays absolute predictions obtained with MEPs@NLO QCD merging (green) and its extension to NLO QCD+EW accuracy including (red) or excluding (black) mixed Born contributions to $V+2$ jet topologies (LO mix). Relative corrections with respect to MEPS@NLO QCD are shown in the lower panels. The bands correspond to scale variations, and in the case of ratios only the numerator is varied.

effects in phase-space regions with up to two resolved jets. In addition to the settings summarised in (2.5)-(2.10) we set the renormalisation scale according to (5.5), and both factorisation and resummation scales to the core scale defined in (5.6). The remaining free parameter, the merging scale separating the individual jet multiplicities, is set to $Q_{\text {cut }}=20 \mathrm{GeV}$. To estimate the uncertainties of our calculation, we vary the renormalisation and factorisation scales by a factor two in a correlated way. The resummation and merging scales are not varied here as they give rise to much smaller uncertainties for the observables to be studied in this paper. While this observation has already been made in various studies based on the MEPs@NLO method [97, 107-112], in appendix B we show that it holds true also in the multi-TeV regime, where the gap between the merging scale and the hard scattering energy can reach two orders of magnitude. The presented analysis has been implemented in RIVET [113].

The first observable we study is the transverse momentum of the reconstructed $\mathrm{W}$ boson in $\ell^{-} \bar{\nu}_{\ell}+$ jets production, as detailed in figure 20 . This observable receives significant contributions from two-jet topologies, which are, however, typically dominated by a first hard jet, while the second jet tends to be much softer. For this reason we observe a rather similar behaviour of NLO QCD+EW effects in fixed-order calculations for $\ell^{-} \bar{\nu}_{\ell}+$ 1 jet (figure 5 ) and $\ell^{-} \bar{\nu}_{\ell}+2$ jets (figure 6 ), as well as in their combination through exclusive sums (figure 15) and with MEPs@NLO QCD+EW virt merging (figure 20). More precisely, 

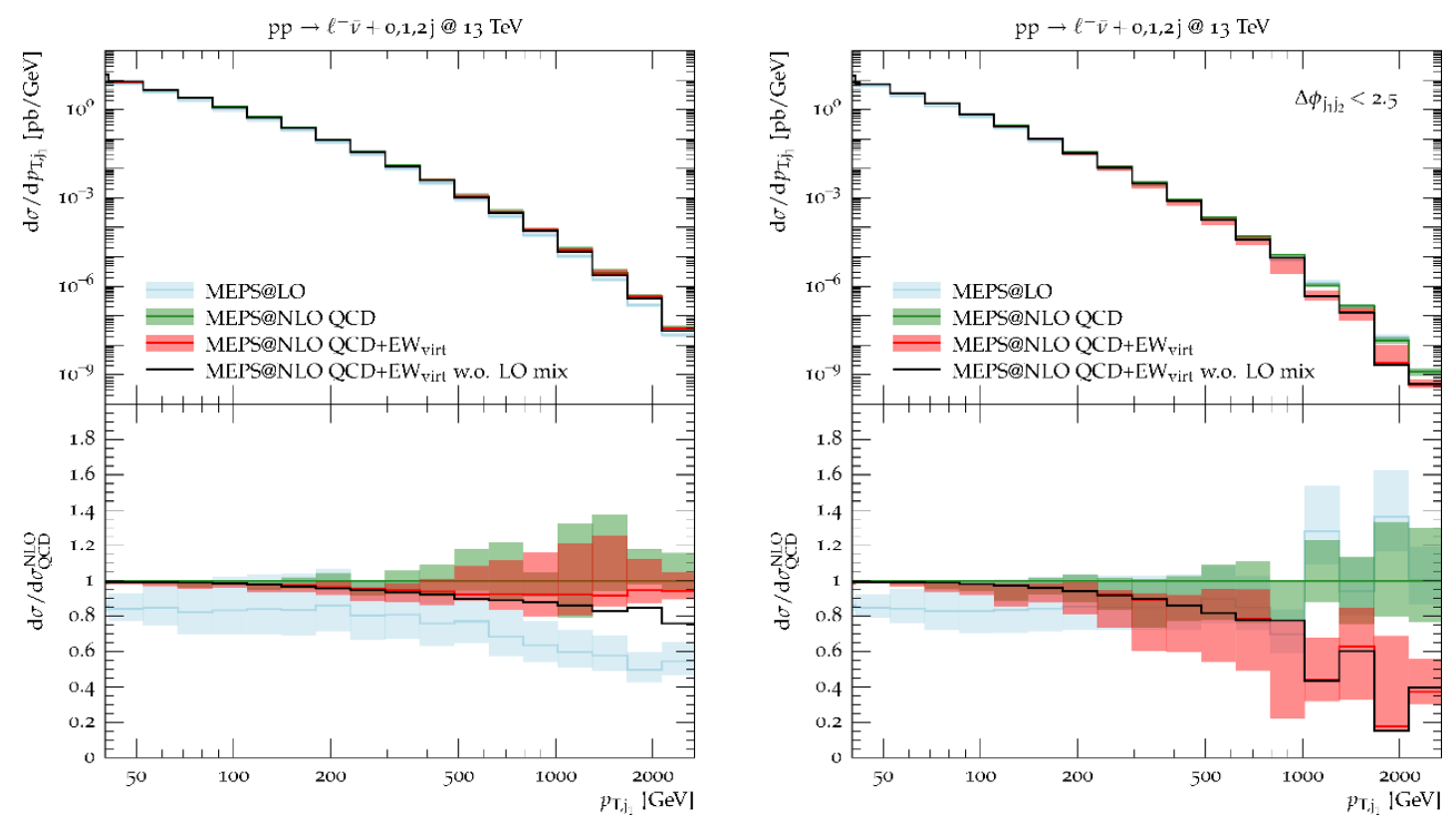

Figure 21. Distributions in the transverse momentum of the hardest jet with standard cuts (left) and in presence of an extra cut $\Delta \phi_{j_{1} j_{2}}<2.5$ (right). Curves and bands as in figure 20.

apart from statistical fluctuations and minor differences due to different scale choices, MEPS@NLO QCD+EW virt predictions are in good agreement with $\ell^{-} \bar{\nu}_{\ell}+2$-jet results, both for what concerns the size of electroweak corrections and scale uncertainties. At high $p_{\mathrm{T}}$ the impact of EW effects in the MEPs framework turns out to be remarkably large and can reach $-50 \%$ or more in the multi-TeV region. This is quantitatively consistent with the outcome of the factorised $\mathrm{QCD} \times \mathrm{EW}$ prescription in inclusive $V+$ jet NLO calculations, and clearly more pronounced than what results from the additive combination of $\mathrm{QCD}+\mathrm{EW}$ fixed-order corrections (figure 5). This feature can be attributed to the inclusion of NLO EW effects in two-jet topologies and, to some extent, also in three-jet topologies via NLO matching to the parton shower. For the vector-boson $p_{\mathrm{T}}$ distribution, as already observed at fixed-order NLO, mixed Born contributions are almost negligible, and the exclusion of back-to-back dijet configurations through a $\Delta \phi_{j_{1} j_{2}}<2.5$ cut have little impact on the behaviour of NLO EW effects.

In figure 21 we examine the transverse momentum of the leading jet. As this observable exhibits a strong sensitivity to higher jet multiplicities - in particular to topologies with two hard back-to-back jets - it is ideally suited to be calculated using a consistent multijet merging. In particular, similarly as for the case of exclusive sums discussed in section 5.1, thanks to the inclusion of dijet topologies as genuine $\ell^{-} \bar{\nu}_{\ell}+2$ jet production processes at NLO, the MEPs@NLO methodology allows one to avoid giant $K$-factors and cures the pathological behaviour of EW corrections observed in fixed-order NLO QCD+EW calculations for $V+1$ jet. Moreover, at sufficient hardness of both jets, the $V+2$ jet configurations are treated as a $V$ boson radiated from a dijet core process, and the scales are set accordingly, further helping to achieve a more physical description of such states. 

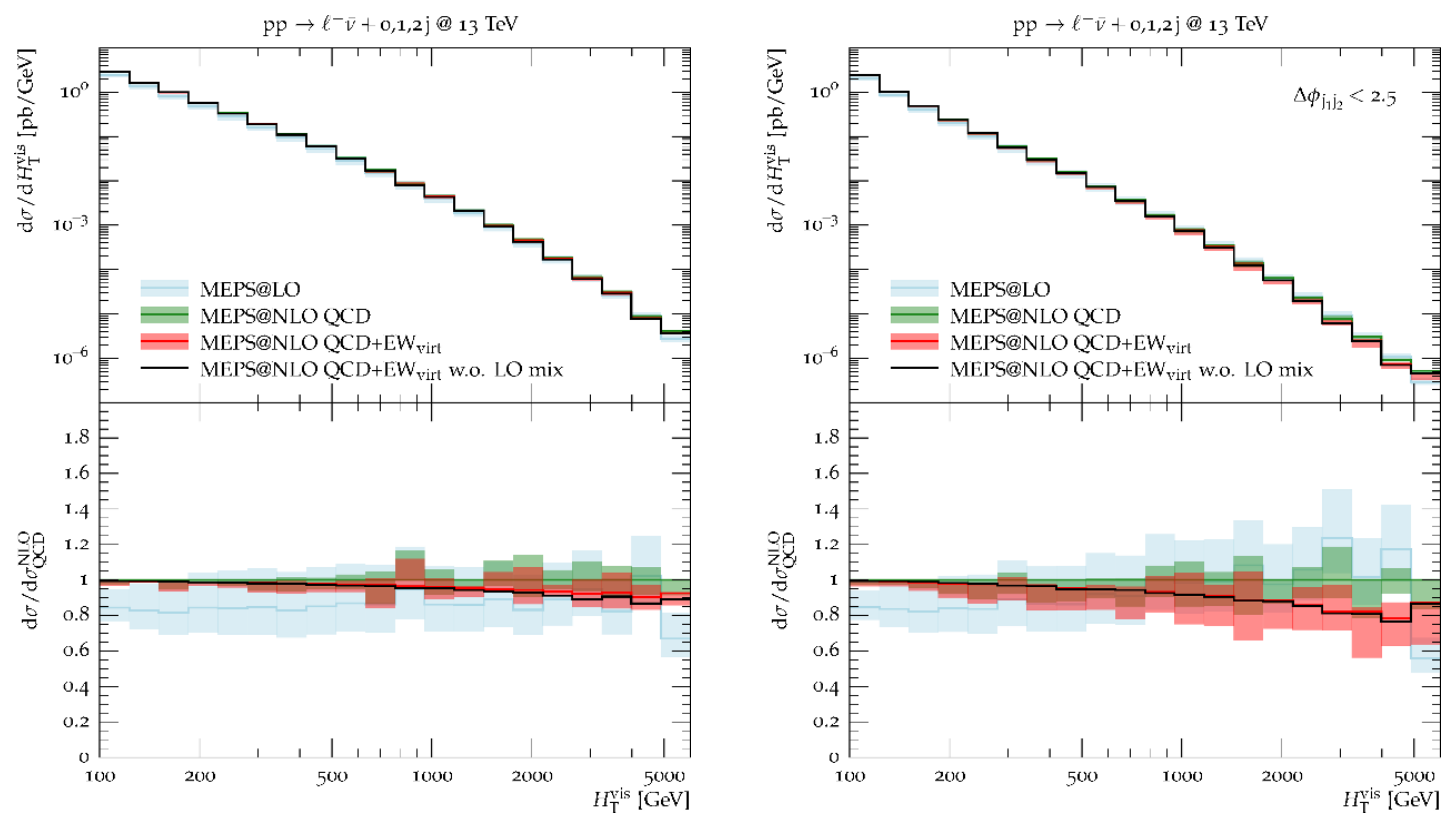

Figure 22. Differential distribution in the total visible transverse energy $H_{\mathrm{T}}^{\mathrm{vis}}=\sum_{i} p_{\mathrm{T}, j_{i}}+\sum_{\ell} p_{\mathrm{T}, \ell}$ with standard cuts (left) and in presence of an extra cut $\Delta \phi_{j_{1} j_{2}}<2.5$ (right). Curves and bands as in figure 20.

This is confirmed by the decent behaviour of NLO QCD scale uncertainties and NLO EW corrections in figure 21. It is worth noting the sizable impact of mixed Born contributions, qualitatively similar to that seen in figure 14, in the tail of this distribution. The absolute size of this effect differs, however, due to the different scale choices. Once back-to-back dijet topologies are removed through a $\Delta \phi_{j_{1} j_{2}}$ cut, as shown on the right hand side of figure 21, mixed Born contributions are suppressed, and the pure Sudakov-type behaviour of the NLO EW corrections is recovered.

Finally, in figure 22 we examine the distribution in the scalar sum of the transverse momenta of all visible objects, i.e. jets and leptons, $H_{\mathrm{T}}^{\text {vis }}$. Again, this is a typical example of an observable receiving contributions from various jet multiplicities simultaneously, and is thus expected to benefit from a multijet merging approach. As the $p_{\mathrm{T}}$ of the leading jet is a major contributor to $H_{\mathrm{T}}^{\mathrm{vis}}$, this observable exhibits many of the characteristics of $p_{\mathrm{T}, j_{1}}$, albeit in reduced severity. Nonetheless, due to the MEPs@NLO approach the troublesome configurations dominated by a hard dijet system are again rendered benign, and the NLO QCD and NLO EW corrections behave as expected. Subleading Born contributions have a visible, but much smaller impact than for the transverse momentum of only the leading jet. As before, the inclusion of a $\Delta \phi_{j_{1} j_{2}}$ cut tends to enhance negative EW correction effects in the tail.

In all investigated observables MEPs@LO predictions are in fairly good agreement with corresponding MEPS@NLO predictions at the NLO QCD level. In particular, due to the scale choice of (5.5) shapes of all differential distributions receive moderate QCD corrections, even in the inclusive $p_{\mathrm{T}}$ distribution of the hardest jet. 


\section{Summary and conclusions}

The inclusion of QCD and EW higher-order corrections in theoretical simulations is a central prerequisite for precision tests of the Standard Model and for new-physics searches at the energy frontier during Run-II of the LHC. In the TeV range, electroweak Sudakov logarithms change the shape of important kinematic distributions significantly and often yield corrections that largely exceed the intrinsic uncertainties of NLO QCD predictions. The recently achieved automation of NLO EW corrections within the SHERPA+OpEnLoops and Munich+OpenLoops Monte Carlo frameworks opens the door to access high precision at the energy frontier for a multitude of processes.

One example where both QCD and EW radiative corrections are large is the experimentally very important process class of vector-boson production in association with jets. Here, it is well known that the inclusive production in conjunction with at least one hard jet is highly sensitive to multijet radiation. In particular, in the regime of high jet $p_{\mathrm{T}}$, fixed-order NLO calculations for inclusive $V+1$ jet production are plagued by giant QCD $K$-factors, and also EW corrections behave in a pathological way. Precise theoretical predictions can only be achieved beyond NLO or via a merging of higher jet multiplicities. In this paper we have developed an approximate framework for multijet merging at NLO including QCD and EW corrections. As a first application, we have presented an inclusive simulation of $V+$ jets production that guarantees NLO QCD+EW accuracy for final states involving zero, one and two jets.

As a prerequisite for the described merging we have calculated NLO QCD+EW fixedorder results for $p p \rightarrow V+2$ jets presenting, for the first time, predictions that describe the off-shell production and decay of all electroweak vector bosons, $V=\mathrm{W}^{ \pm}, Z / \gamma^{*}$, including all possible final states with charged leptons and neutrinos. Off-shell and $Z / \gamma^{*}$ interference effects were included throughout by means of an automated implementation of the complex-mass scheme at NLO. Detailed NLO QCD+EW predictions are provided for various important kinematic observables, including the $p_{\mathrm{T}}$ spectra of the leptons, the reconstructed vector bosons and the accompanying jets. The tails of such distributions receive large EW corrections of Sudakov type, which can reach $-40 \%$ at $2 \mathrm{TeV}$ and are maximally pronounced in $V+2$ jet configurations where the leading transverse momentum is carried by the vector boson. As expected, such large Sudakov corrections are hardly affected by the leptonic decays, whereas less inclusive observables, in particular the invariant mass of the lepton pair in $Z / \gamma^{*} \rightarrow \ell^{+} \ell^{-}$, exhibit a strong dependence on genuine QED bremsstrahlung, with corrections of up to $50 \%$. Besides NLO EW corrections, we have also studied all subleading Born and photon-induced $V+2$ jet processes which can give sizeable contributions in the $\mathrm{TeV}$ range.

Towards a merging including EW corrections we first combined $V+1$ jet and $V+2$ jet NLO QCD+EW predictions by means of an implementation of naïve exclusive sums. Already here the perturbative convergence was found to be largely stabilized. Within the context of exclusive sums, we have developed an NLO EW approximation that combines exact EW virtual corrections with an inclusive treatment of bremsstrahlung effects. In the kinematic regime dominated by large virtual Sudakov corrections, the agreement with 
respect to the full calculation was found to be mostly at the few percent level. This approximation allowed us to include NLO EW corrections into the MEPS@NLO multijet merging framework of SHERPA in a straightforward way. Within the MEPS@NLO framework we have provided multijet-merged predictions for $\ell^{-} \bar{\nu}_{\ell}+$ jets production including QCD $+\mathrm{EW}$ corrections up to two jets. Thanks to the inclusion of dijet topologies as genuine $\ell^{-} \bar{\nu}_{\ell}+2$ jet production processes at NLO, the MEPS@NLO methodology allowed us to stabilize the perturbative convergence and to cure the pathological behaviour of EW corrections observed in fixed-order NLO QCD+EW calculations for $V+1$ jet.

In a forthcoming paper, we plan to investigate multijet-merged cross-section ratios for different $V+$ jets processes, including a thorough study of theoretical uncertainties. Precise predictions for such ratios including EW corrections can reduce important systematic uncertainties in monojet searches for dark matter and many other BSM searches, resulting in significant improvements of the experimental sensitivity.

The findings presented here motivate similar studies for a wide range of other Standard Model processes, and at the same time further developments towards a complete partonshower matching and multi-jet merging at NLO QCD+EW.

\section{Acknowledgments}

We thank A. Denner, S. Dittmaier and L. Hofer for providing us with the one-loop tensorintegral library Collier. We also want to thank A. Denner for providing us detailed numerical results of [45], and for useful discussions concerning the complex-mass scheme. This research was supported in part by the Swiss National Science Foundation (SNF) under contract PP00P2-128552 and by the Research Executive Agency (REA) of the European Union under the Grant Agreements PITN-GA-2010-264564 (LHCPhenoNet), PITN-GA2012-315877 (MCnet) and PITN-GA-2012-316704 (HiggsTools).

\section{A Further NLO predictions for $V+2$ jet production}

In figures 23-24 we present further fixed-order NLO QCD+EW results for $p p \rightarrow \ell^{+} \nu_{\ell}+$ 2 jets, $p p \rightarrow \ell^{-} \bar{\nu}_{\ell}+2$ jets, $p p \rightarrow \ell^{+} \ell^{-}+2$ jets and $p p \rightarrow \nu_{\ell} \bar{\nu}_{\ell}+2$ jets at the LHC. In particular, we show distributions in $H_{\mathrm{T}}^{\text {tot }}$ and the invariant mass of the two leading jets, $m_{\mathrm{j}_{1} \mathrm{j}_{2}}$. In the tail of the $H_{\mathrm{T}}^{\text {tot }}$ distribution, NLO QCD and EW corrections approach the $70 \%$ and $10 \%$ level, respectively, and the $\mathrm{QCD} \times \mathrm{EW}$ curve suggests that due to the sizeable QCD corrections the importance of NLO EW corrections is underestimated by about a factor two in the NLO QCD+EW prediction. The corrections in $m_{\mathrm{j}_{1} \mathrm{j}_{2}}$ show a very different picture. Here, NLO EW corrections are very small and almost completely independent of the dijet mass up to the multi-TeV range. However, in this regime, LO EW contributions from $V+2$ jet production via vector-boson fusion will become sizable. Thus, a detailed study of EW corrections in $m_{\mathrm{j}_{1} \mathrm{j}_{2}}$ requires the inclusion of the subleading one-loop corrections of $\mathcal{O}\left(\alpha_{\mathrm{S}} \alpha^{4}\right)$ and $\mathcal{O}\left(\alpha^{5}\right)$. 

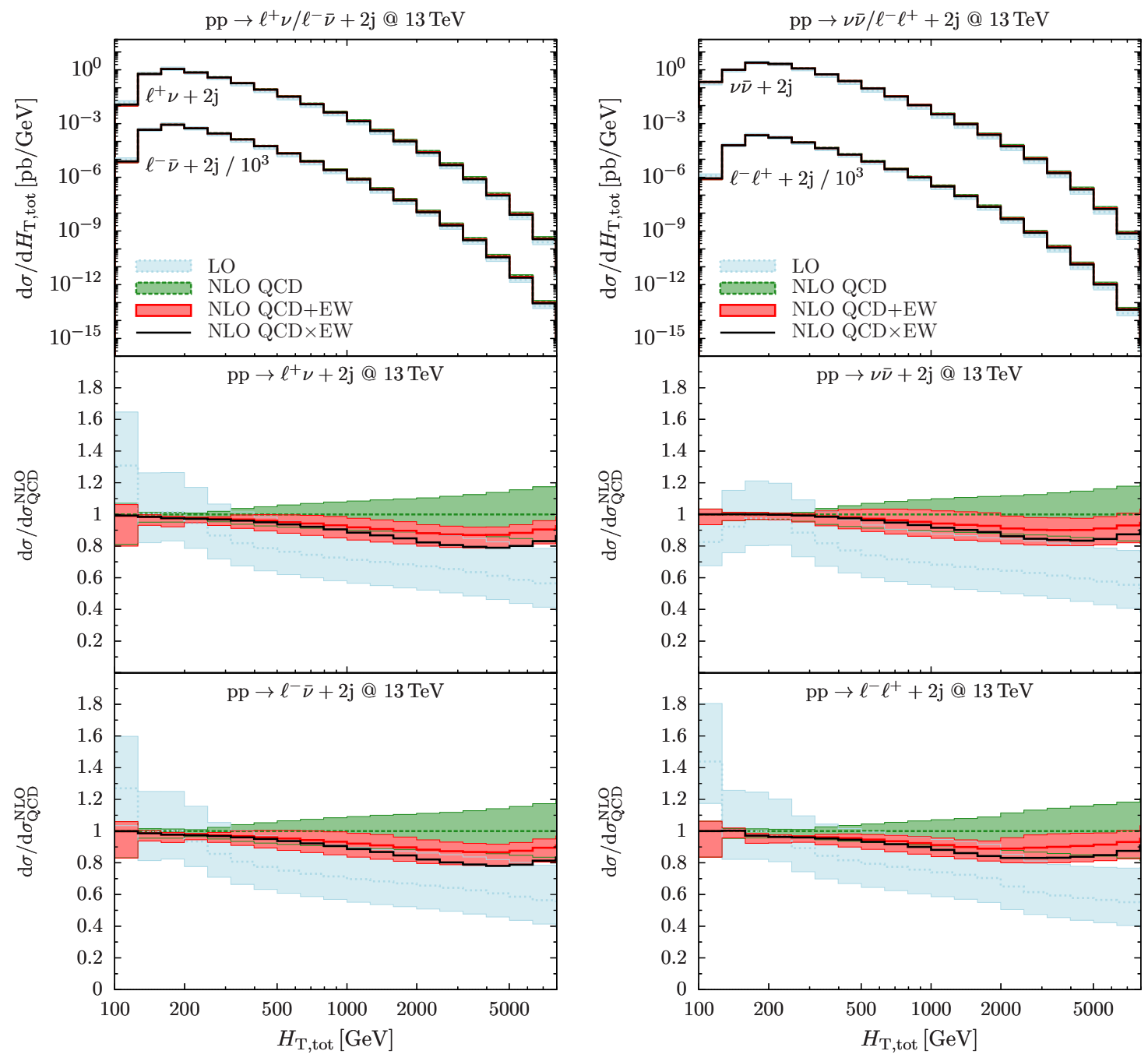

Figure 23. Differential distribution in $H_{\mathrm{T}}^{\text {tot }}=\sum_{k} p_{T, j_{k}}+\sum_{\ell} p_{T, \ell}+E_{\mathrm{T}}$. Curves and bands as in figure 5 .

\section{B Multijet merging systematic uncertainties in the $\mathrm{TeV}$ range}

This appendix aims at quantifying uncertainties associated with the merging scale dependence of the results presented in section 5.5. This dependence has never been studied in the literature for observables in the multi-TeV range, where the combination of very high energies and a small merging scale could result in large spurious logarithms. Indeed, the power counting of $[54,55]$ shows that inclusive MEPS predictions involve uncancelled logarithms of the generic form

$$
\text { MEPS@Lo : } \frac{1}{N_{C}} \alpha_{\mathrm{S}} \log \frac{\mu_{Q}}{Q_{\text {cut }}} \quad \text { and } \quad \text { MEPS@NLO : } \frac{1}{N_{C}} \alpha_{\mathrm{S}}^{2} \log ^{3} \frac{\mu_{Q}}{Q_{\text {cut }}},
$$

where $\mu_{Q}$ and $Q_{\text {cut }}$ denote the resummation and the merging scale, respectively. They arise in $n$-jet observables as a result of the partitioning of the phase space of the extra emission in an unresolved region $\left(Q_{n+1}<Q_{\text {cut }}\right)$ and a resolved region $\left(Q_{n+1}>Q_{\text {cut }}\right)$, 

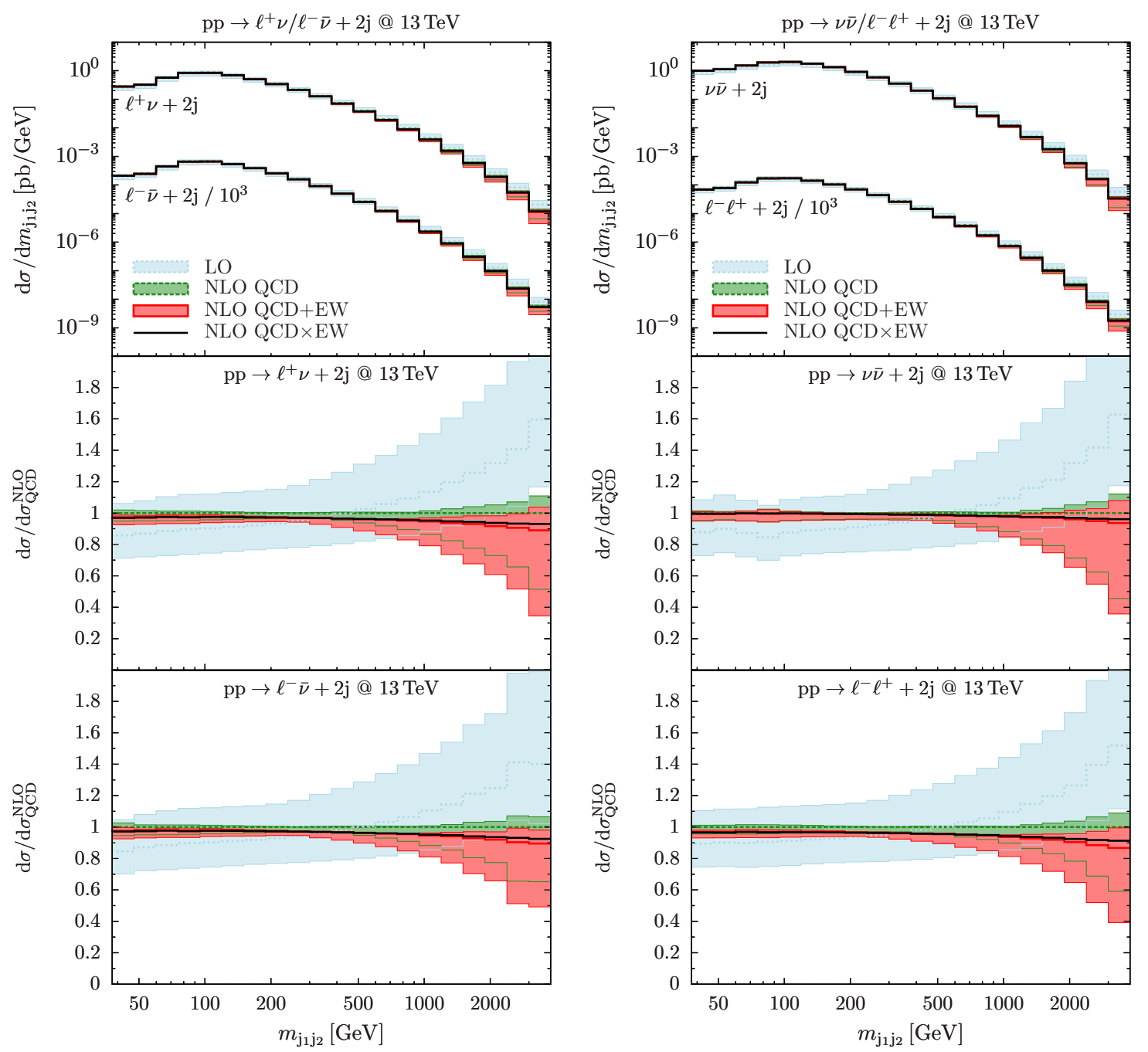

Figure 24. Differential distributions in the invariant mass $m_{\mathrm{j}_{1} \mathrm{j}_{2}}$ of the two hardest jets. Curves and bands as in figure 5 .

which are described, respectively, in terms of $n$-jet and $n+1$-jet (N)LO matrix elements combined with the parton shower in the MEPs approach. When the $(n+1)$-th emission is integrated out, the logarithms of $Q_{\text {cut }}$ that originate from both regions cancel to a large extent, but the limited logarithmic accuracy of the parton shower results in left-over contributions of type (B.1). For moderate values of $\mu_{Q} / Q_{\text {cut }}$ their impact is small. However, when requiring either a vector boson or a jet of $1 \mathrm{TeV}$ transverse momentum in inclusive $V+$ jets production, $\mu_{Q}$ takes values of a comparable scale, and the numerical value of the uncancelled logarithms (B.1) could in principle exceed the size of renormalisation and factorisation scale variations at NLO, thereby spoiling the claimed accuracy.

Such a scenario is clearly excluded by the quantitative analysis of the $Q_{\text {cut }}$ dependence of our predictions presented in figures $25-27$ for the case of $W+$ jets production. Figure 25 displays MEPs@LO and MEPS@NLO predictions for the differential $0 \rightarrow 1$ and $1 \rightarrow 2$ jet- $k_{\perp}$ resolution scales, $d_{01}$ and $d_{12}$, which represent the most sensitive observables to merging 

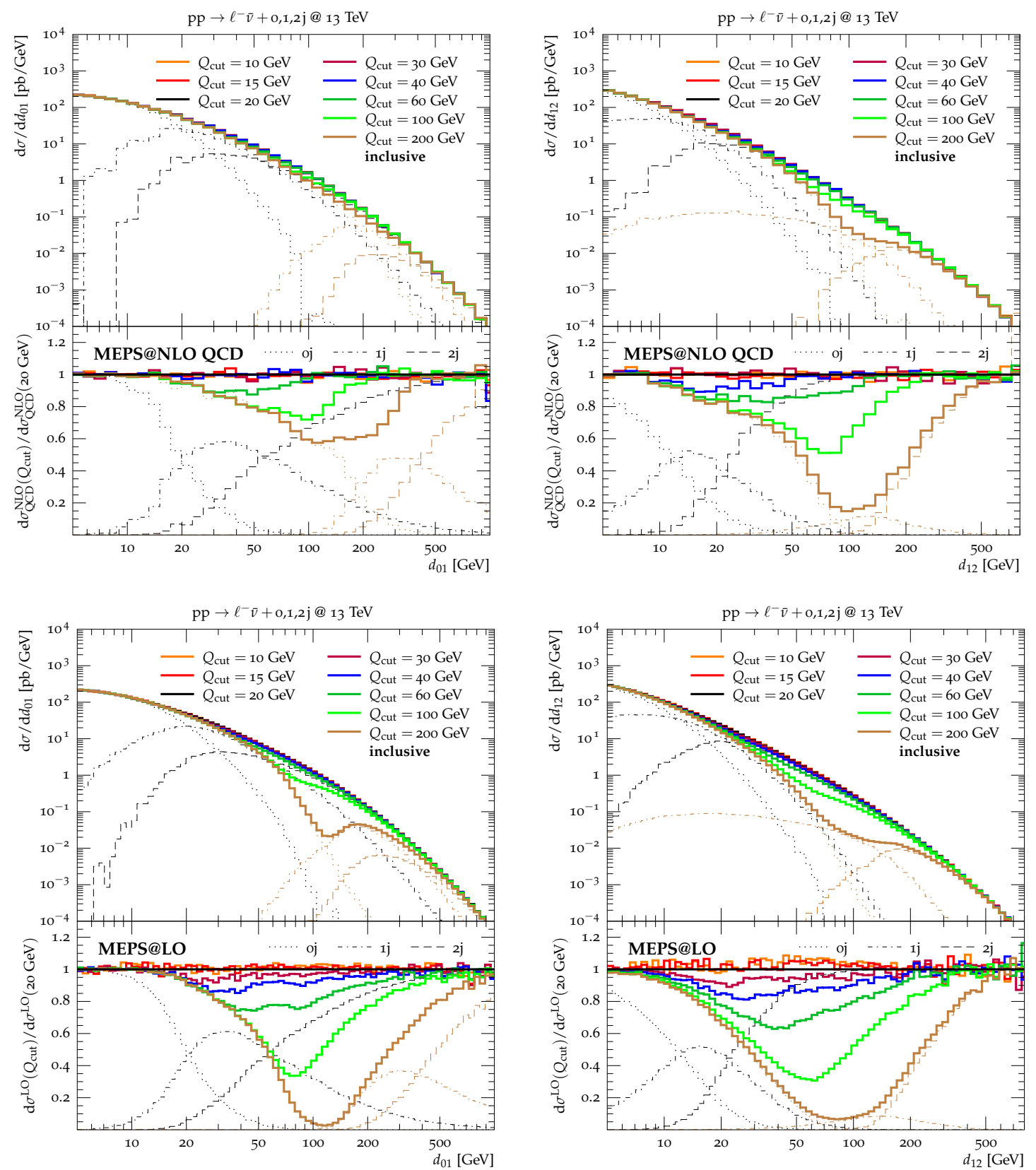

Figure 25. Multijet merging systematics of the $1 \rightarrow 0$ (left) and $2 \rightarrow 1$ (right) $k_{\perp}$ jet resolutions $(R=0.6)$ in $p p \rightarrow \ell^{-} \bar{\nu}+$ jets events in leading order (bottom) and next-to-leading order (top) multijet merging in the MEPS scheme. Only basic lepton acceptance cuts are applied. The contributions of the individual jet multiplicities are indicated by dotted, dashdotted and dashed lines for $Q_{\text {cut }}=20$ and $200 \mathrm{GeV}$. 

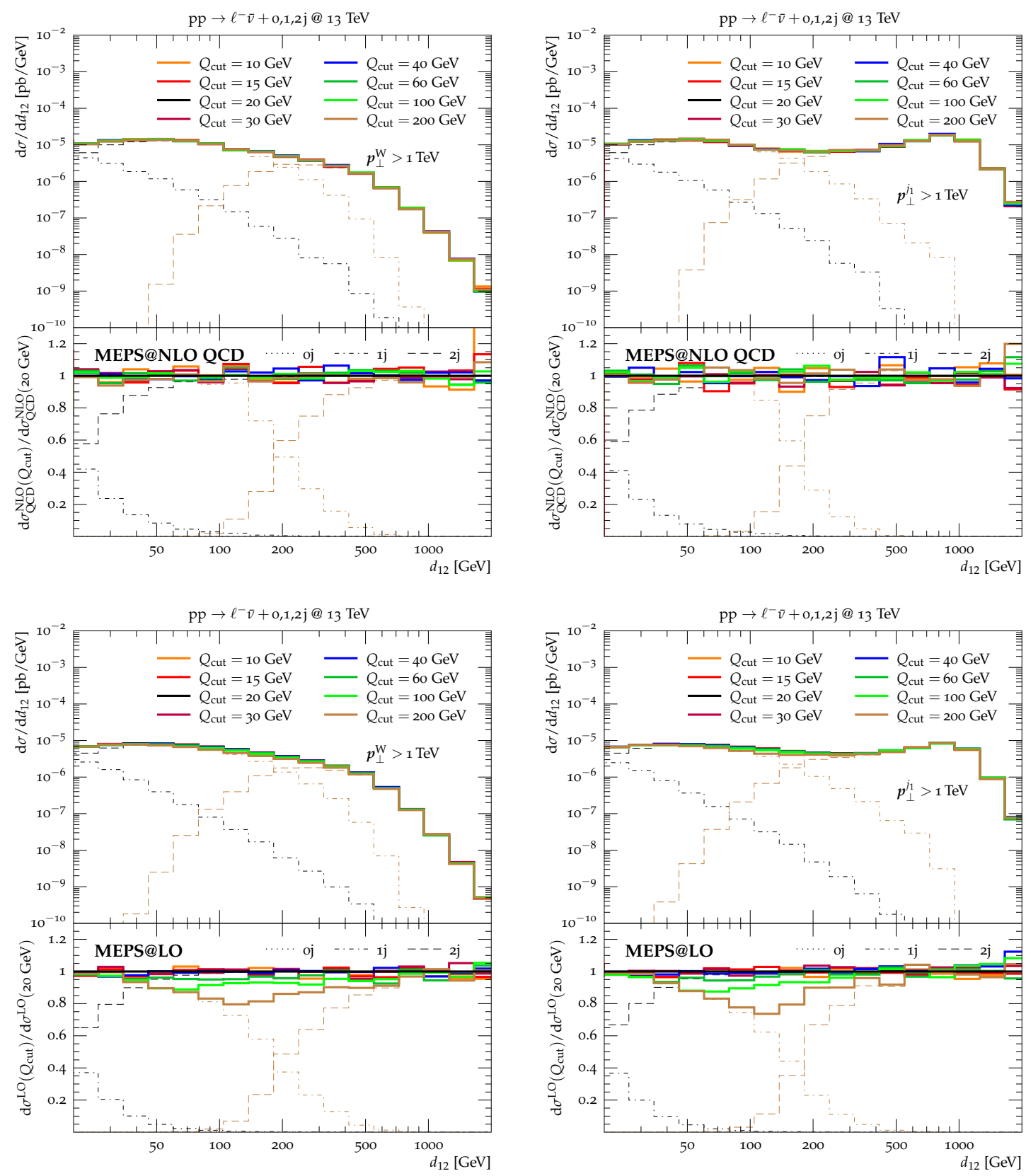

Figure 26. Multijet merging systematics of the $2 \rightarrow 1 k_{\perp}$ jet resolution $(R=0.6)$ in $p p \rightarrow \ell^{-} \bar{\nu}+$ jets in events with a reconstructed $W$ boson (left) or leading jet (right) with $p_{\perp}>1 \mathrm{TeV}$ in leading order (bottom) and next-to-leading order (top) multijet merging in the MEPs scheme. Only basic lepton acceptance cuts are applied. The contributions of the individual jet multiplicities are indicated by dotted, dashdotted and dashed lines for $Q_{\text {cut }}=20$ and $200 \mathrm{GeV}$. 

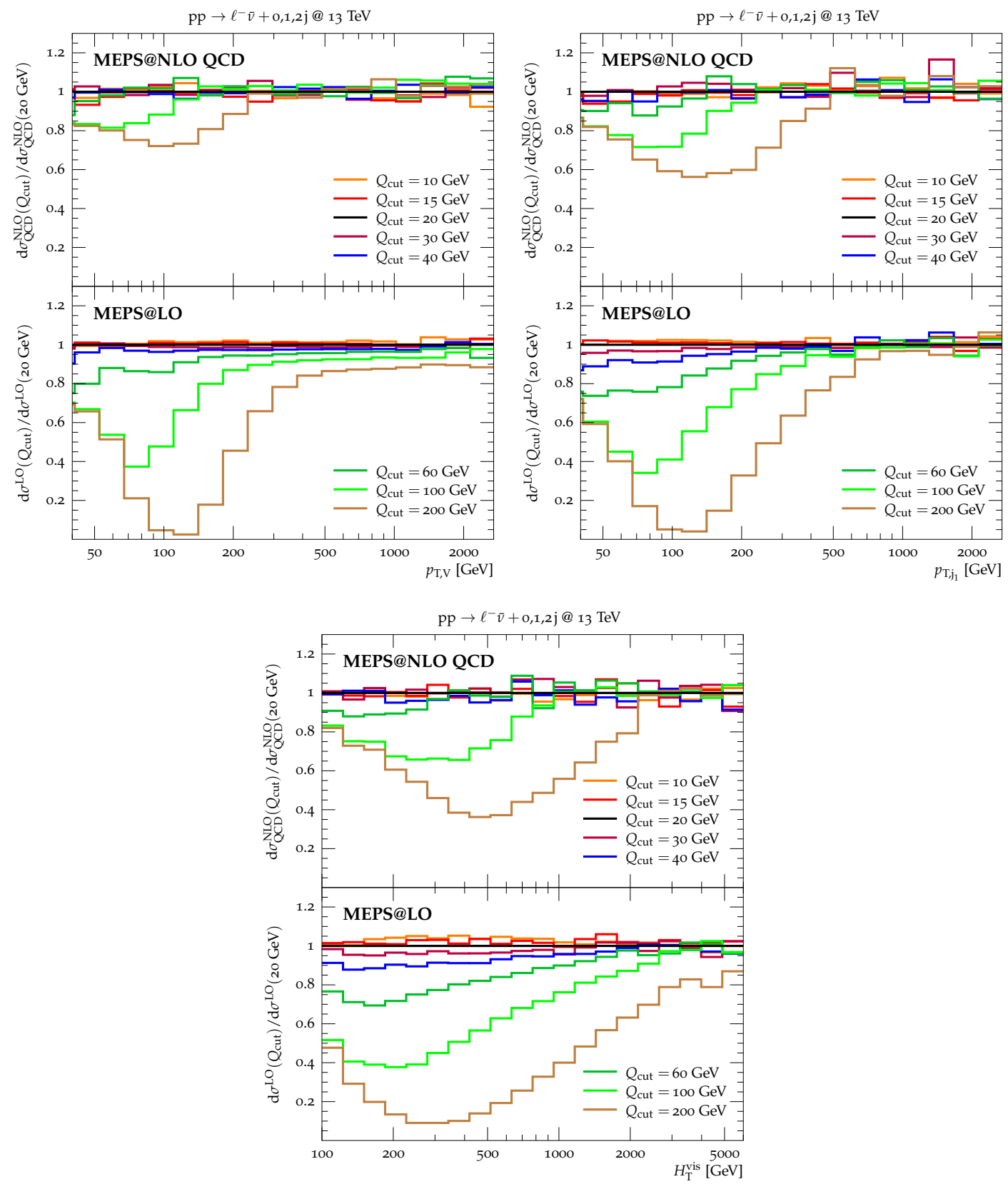

Figure 27. Multijet merging systematics for transverse momentum of the reconstructed vector boson(top left), the hardest jet (top right) and the total visible transverse energy $H_{\mathrm{T}}^{\mathrm{vis}}$ (bottom) with standard cuts. 
effects. They can be regarded as the relative transverse momenta associated with the emissions of the hardest and second-hardest jet, respectively. The plots show the sensitivity with respect to variations of the merging scale in a very wide range, from 10 to $200 \mathrm{GeV}$. In the phase space region below the minimum $Q_{\text {cut }}$, we observe that all computations are in good mutual agreement. This is due to the fact that, by construction, in this region the $n \rightarrow n+1$ jet resolution scale always corresponds to a parton shower emission matched to $n$-jet $(\mathrm{N}) \mathrm{LO}$ matrix elements.

In the regions where $d_{01}$ and $d_{12}$ are between the minimum and the maximun $Q_{\text {cut }}$ one can see a significant sensitivity to the merging scale. More precisely, predictions with $Q_{\text {cut }} \in[10,30] \mathrm{GeV}$ are very stable, while increasing $Q_{\text {cut }}$ beyond $30 \mathrm{GeV}$ gives rise to increasingly pronounced and wide dips centered at $d_{i j} \sim Q_{\text {cut }}$. This is due to the fact that in the region above $30 \mathrm{GeV}$ the parton shower's soft-collinear approximation ceases to be a sufficiently good description. This feature is clearly more pronounced in MEPs@Lo, where the emission is entirely given by the parton shower, while the problem is significantly alleviated in MEPs@NLO, where the shower emission below $Q_{\text {cut }}$ is matched to tree-level matrix elements. Nevertheless, merging scales well beyond $50 \mathrm{GeV}$ start to be problematic also for NLO merging. In this regime, it is important to realise that $Q_{\text {cut }}$ variations in MEPs@NLO (MEPs@LO) amount to a comparison of LO+PS versus NLO+PS (PS versus LO+PS) descriptions, where the latter are clearly superior. Thus, $Q_{\text {cut }}$ should be chosen as small as computationally feasible. Note also that, in principle, the deficit of the parton shower in the intermediate $k_{\perp}$ regions could be attenuated by increasing the resummation scale $\mu_{Q}$. However this would alter the resummation of large logarithms in the region of very small $k_{\perp}$. Thus, in order to avoid large shower uncertainties, it is preferable to keep $Q_{\text {cut }}$ below $30-40 \mathrm{GeV}$ in MEPs@NLO . In this case the $d_{01}$ and $d_{12}$ distributions turn out to be very robust with respect to $Q_{\text {cut }}$ variations in the whole range from $\mathcal{O}(1 \mathrm{GeV})$ to $\mathcal{O}(1 \mathrm{TeV})$.

The $Q_{\text {cut }}$ sensitivity in the $\mathrm{TeV}$ region is investigated in more detail in figure 26, which shows distributions in the $1 \rightarrow 2 k_{\perp}$ resolution scale in presence of a lower cut of $1 \mathrm{TeV}$ on the transverse momentum of the $W$ boson or, alternatively, of the first jet. We find that the $d_{12}$ spectra are remarkably stable - especially in MEPs@NLO but also in MEPs@LOwith respect to $Q_{\text {cut }}$ variation from 10 to $200 \mathrm{GeV}$, i.e. in a region where $\mu_{Q}$ and $Q_{\text {cut }}$ differ by up to two orders of magnitude.

This high quality of the MEPs@NLO merging procedure is due, among other things, to the fact that the implementation of Sudakov effects through the truncated vetoed parton shower guarantees exactly the same logarithmic resummation on both sides of the merging cut. In the domain above $Q_{\text {cut }}$ the NLO calculation supplements fixed-order terms beyond the parton shower accuracy, which result, upon integration, in potentially troubling terms of the type (B.1). The fact that these extra contributions remain comparably small can be understood by considering the de facto quality of logarithmic resummation in the parton shower despite its formally limited accuracy. For instance, although not fully under control, subleading colour NLL contributions are largely captured by replacing $\left.\left.C_{A / F}\right|_{N_{C} \rightarrow \infty} \rightarrow C_{A / F}\right|_{N_{C}=3}$ in the parton shower's resummation, otherwise performed in the $N_{C} \rightarrow \infty$-limit. Further, the usage of CMW scales in the parton shower [114] in- 
cludes dominant contributions of NNLL accuracy through the running of $\alpha_{\mathrm{S}}$, giving a good numerical reproduction of higher logarithmic terms.

Finally, figure 27 examines the $Q_{\text {cut }}$-dependence of the observables studied in section 5.5, now integrating over additional emissions. The uncertainties displayed here are dominated by statistical fluctuations for a reasonable variation in the range of $Q_{\text {cut }} \in[10,40] \mathrm{GeV}$. Taking these fluctuations into account the merging systematics are on a level of $5 \%$ and are thus not included in the uncertainty estimate of section 5.5. As can be seen, if only the $\mathrm{TeV}$ range is to be studied, $Q_{\text {cut }}$ values of up to $200 \mathrm{GeV}$ can be chosen without introducing a large uncertainty in the results. However, only a small merging cut as the one used in section 5.5 ensures a reliable prediction for the whole energy range.

Open Access. This article is distributed under the terms of the Creative Commons Attribution License (CC-BY 4.0), which permits any use, distribution and reproduction in any medium, provided the original author(s) and source are credited.

\section{References}

[1] ATLAS collaboration, Measurement of the production cross section for W-bosons in association with jets in pp collisions at $\sqrt{s}=7 \mathrm{TeV}$ with the ATLAS detector, Phys. Lett. B 698 (2011) 325 [arXiv: 1012.5382] [INSPIRE].

[2] ATLAS collaboration, Study of jets produced in association with a $W$ boson in pp collisions at $\sqrt{s}=7$ TeV with the ATLAS detector, Phys. Rev. D 85 (2012) 092002 [arXiv:1201.1276] [INSPIRE].

[3] ATLAS collaboration, Measurements of the $W$ production cross sections in association with jets with the ATLAS detector, Eur. Phys. J. C 75 (2015) 82 [arXiv:1409.8639] [InSPIRE].

[4] CMS collaboration, Jet production rates in association with $W$ and $Z$ bosons in $p p$ collisions at $\sqrt{s}=7 \mathrm{TeV}$, JHEP 01 (2012) 010 [arXiv:1110.3226] [INSPIRE].

[5] CMS collaboration, Study of the dijet mass spectrum in $p p \rightarrow W+$ jets events at $\sqrt{s}=7$ TeV, Phys. Rev. Lett. 109 (2012) 251801 [arXiv:1208.3477] [INSPIRE].

[6] CMS collaboration, Differential cross section measurements for the production of a $W$ boson in association with jets in proton-proton collisions at $\sqrt{s}=7$ TeV, Phys. Lett. B 741 (2015) 12 [arXiv: 1406.7533] [INSPIRE].

[7] P.B. Arnold and M.H. Reno, The complete computation of high $p_{T} W$ and $Z$ production in 2nd order QCD, Nucl. Phys. B 319 (1989) 37 [Erratum ibid. B 330 (1990) 284] [InSPIRE].

[8] P.B. Arnold, R.K. Ellis and M.H. Reno, High $p_{T} W$ and $Z$ production at the Tevatron, Phys. Rev. D 40 (1989) 912 [INSPIRE].

[9] J.M. Campbell and R.K. Ellis, Next-to-leading order corrections to $W+2$ jet and $Z+2$ jet production at hadron colliders, Phys. Rev. D 65 (2002) 113007 [hep-ph/0202176] [INSPIRE].

[10] F. Febres Cordero, L. Reina and D. Wackeroth, NLO QCD corrections to $W$ boson production with a massive b-quark jet pair at the Tevatron $p \bar{p}$ collider, Phys. Rev. D 74 (2006) 034007 [hep-ph/0606102] [INSPIRE].

[11] J.M. Campbell et al., Associated production of a $W$ boson and one $B$ jet, Phys. Rev. D 79 (2009) 034023 [arXiv:0809.3003] [INSPIRE]. 
[12] R.K. Ellis, Z. Kunszt, K. Melnikov and G. Zanderighi, One-loop calculations in quantum field theory: from Feynman diagrams to unitarity cuts, Phys. Rept. 518 (2012) 141 [arXiv:1105.4319] [INSPIRE].

[13] H. Ita, Susy theories and QCD: numerical Approaches, J. Phys. A 44 (2011) 454005 [arXiv:1109.6527] [INSPIRE].

[14] C.F. Berger et al., Precise predictions for $W+3$ jet production at hadron colliders, Phys. Rev. Lett. 102 (2009) 222001 [arXiv:0902.2760] [INSPIRE].

[15] R.K. Ellis, K. Melnikov and G. Zanderighi, Generalized unitarity at work: first NLO QCD results for hadronic $W+3$ jet production, JHEP 04 (2009) 077 [arXiv:0901.4101] [INSPIRE].

[16] R.K. Ellis, K. Melnikov and G. Zanderighi, $W+3$ jet production at the Tevatron, Phys. Rev. D 80 (2009) 094002 [arXiv: 0906.1445] [INSPIRE].

[17] C.F. Berger et al., Next-to-leading order QCD predictions for $W+3$-jet distributions at hadron colliders, Phys. Rev. D 80 (2009) 074036 [arXiv:0907.1984] [INSPIRE].

[18] C.F. Berger et al., Precise predictions for $W+4$ jet production at the large hadron collider, Phys. Rev. Lett. 106 (2011) 092001 [arXiv: 1009.2338] [INSPIRE].

[19] Z. Bern et al., Next-to-leading order W+5-jet production at the LHC, Phys. Rev. D 88 (2013) 014025 [arXiv: 1304.1253] [INSPIRE].

[20] R. Boughezal, C. Focke, X. Liu and F. Petriello, $W$-boson production in association with a jet at next-to-next-to-leading order in perturbative QCD, Phys. Rev. Lett. 115 (2015) 062002 [arXiv: 1504.0213] [INSPIRE].

[21] A. Gehrmann-De Ridder et al., Precise QCD predictions for the production of a $Z$ boson in association with a hadronic jet, arXiv:1507.0285.

[22] V.S. Fadin, L.N. Lipatov, A.D. Martin and M. Melles, Resummation of double logarithms in electroweak high-energy processes, Phys. Rev. D 61 (2000) 094002 [hep-ph/9910338] [INSPIRE].

[23] J.H. Kuhn, A.A. Penin and V.A. Smirnov, Summing up subleading Sudakov logarithms, Eur. Phys. J. C 17 (2000) 97 [hep-ph/9912503] [inSPIRE].

[24] A. Denner and S. Pozzorini, One loop leading logarithms in electroweak radiative corrections. 1. Results, Eur. Phys. J. C 18 (2001) 461 [hep-ph/0010201] [INSPIRE].

[25] A. Denner and S. Pozzorini, One loop leading logarithms in electroweak radiative corrections. 2. Factorization of collinear singularities, Eur. Phys. J. C 21 (2001) 63 [hep-ph/0104127] [INSPIRE].

[26] M. Ciafaloni, P. Ciafaloni and D. Comelli, Bloch-Nordsieck violating electroweak corrections to inclusive TeV scale hard processes, Phys. Rev. Lett. 84 (2000) 4810 [hep-ph/0001142] [INSPIRE].

[27] U. Baur, Weak boson emission in hadron collider processes, Phys. Rev. D 75 (2007) 013005 [hep-ph/0611241] [INSPIRE].

[28] K. Mishra et al., Electroweak corrections at high energies, arXiv:1308.1430 [INSPIRE].

[29] J.H. Kuhn, A. Kulesza, S. Pozzorini and M. Schulze, Logarithmic electroweak corrections to hadronic $Z+1$ jet production at large transverse momentum, Phys. Lett. B 609 (2005) 277 [hep-ph/0408308] [INSPIRE].

[30] J.H. Kuhn, A. Kulesza, S. Pozzorini and M. Schulze, One-loop weak corrections to hadronic production of $Z$ bosons at large transverse momenta, Nucl. Phys. B 727 (2005) 368 [hep-ph/0507178] [INSPIRE]. 
[31] J.H. Kuhn, A. Kulesza, S. Pozzorini and M. Schulze, Electroweak corrections to hadronic photon production at large transverse momenta, JHEP 03 (2006) 059 [hep-ph/0508253] [INSPIRE].

[32] J.H. Kuhn, A. Kulesza, S. Pozzorini and M. Schulze, Electroweak corrections to large transverse momentum production of $W$ bosons at the LHC, Phys. Lett. B 651 (2007) 160 [hep-ph/0703283] [INSPIRE].

[33] J.H. Kuhn, A. Kulesza, S. Pozzorini and M. Schulze, Electroweak corrections to hadronic production of $W$ bosons at large transverse momenta, Nucl. Phys. B 797 (2008) 27 [arXiv:0708.0476] [INSPIRE].

[34] A. Denner, S. Dittmaier, T. Kasprzik and A. Muck, Electroweak corrections to $W+$ jet hadroproduction including leptonic W-boson decays, JHEP 08 (2009) 075 [arXiv:0906.1656] [INSPIRE].

[35] A. Denner, S. Dittmaier, T. Kasprzik and A. Muck, Electroweak corrections to dilepton + jet production at hadron colliders, JHEP 06 (2011) 069 [arXiv:1103.0914] [INSPIRE].

[36] A. Denner, S. Dittmaier, T. Kasprzik and A. Mück, Electroweak corrections to monojet production at the LHC, Eur. Phys. J. C 73 (2013) 2297 [arXiv:1211.5078] [INSPIRE].

[37] S. Kallweit et al., $N L O Q C D+E W$ automation and precise predictions for $V+$ multijet production, arXiv:1505.0570.

[38] S. Actis, A. Denner, L. Hofer, A. Scharf and S. Uccirati, Recursive generation of one-loop amplitudes in the standard model, JHEP 04 (2013) 037 [arXiv:1211.6316] [INSPIRE].

[39] S. Actis, A. Denner, L. Hofer, A. Scharf and S. Uccirati, EW and QCD one-loop amplitudes with RECOLA, PoS (RADCOR 2013) 034 [arXiv:1311.6662] [INSPIRE].

[40] F. Cascioli, P. Maierhofer and S. Pozzorini, Scattering amplitudes with open loops, Phys. Rev. Lett. 108 (2012) 111601 [arXiv:1111.5206] [INSPIRE].

[41] S. Kallweit, J.M. Lindert, P. Maierhöfer, S. Pozzorini and M. Schönherr, NLO electroweak automation and precise predictions for $W+$ multijet production at the LHC, JHEP 04 (2015) 012 [arXiv: 1412.5157] [INSPIRE].

[42] S. Frixione, V. Hirschi, D. Pagani, H.S. Shao and M. Zaro, Weak corrections to Higgs hadroproduction in association with a top-quark pair, JHEP 09 (2014) 065 [arXiv: 1407.0823] [INSPIRE].

[43] M. Chiesa, N. Greiner and F. Tramontano, Electroweak corrections for LHC processes, arXiv: 1507.0857.

[44] M. Chiesa et al., Electroweak Sudakov corrections to new physics searches at the LHC, Phys. Rev. Lett. 111 (2013) 121801 [arXiv:1305.6837] [INSPIRE].

[45] A. Denner, L. Hofer, A. Scharf and S. Uccirati, Electroweak corrections to lepton pair production in association with two hard jets at the LHC, JHEP 01 (2015) 094 [arXiv: 1411.0916] [INSPIRE].

[46] F. Cascioli, J. Lindert, P. Maierhöfer and S. Pozzorini, OpenLoops one-loop generator, available at http://openloops.hepforge.org.

[47] M. Schönherr, An automated subtraction of NLO EW infrared divergences, in preparation.

[48] T. Gleisberg and F. Krauss, Automating dipole subtraction for QCD NLO calculations, Eur. Phys. J. C 53 (2008) 501 [arXiv:0709.2881] [INSPIRE].

[49] T. Gleisberg et al., Event generation with SHERPA 1.1, JHEP 02 (2009) 007 [arXiv:0811.4622] [INSPIRE]. 
[50] A. Denner, S. Dittmaier, M. Roth and L.H. Wieders, Electroweak corrections to charged-current $e^{+} e^{-} \rightarrow 4$ fermion processes: technical details and further results, Nucl. Phys. B 724 (2005) 247 [Erratum ibid. B 854 (2012) 504] [hep-ph/0505042] [INSPIRE].

[51] M. Rubin, G.P. Salam and S. Sapeta, Giant QCD K-factors beyond NLO, JHEP 09 (2010) 084 [arXiv: 1006.2144] [INSPIRE].

[52] S. Dittmaier, A. Huss and C. Schwinn, Mixed QCD-electroweak $O\left(\alpha_{s} \alpha\right)$ corrections to Drell-Yan processes in the resonance region: pole approximation and non-factorizable corrections, Nucl. Phys. B 885 (2014) 318 [arXiv:1403.3216] [InSPIRE].

[53] S. Dittmaier, A. Huss and C. Schwinn, Dominant mixed QCD-electroweak $O\left(\alpha_{s} \alpha\right)$ corrections to Drell-Yan processes in the resonance region, arXiv:1511.0801.

[54] S. Hoeche, F. Krauss, M. Schönherr and F. Siegert, QCD matrix elements + parton showers: the NLO case, JHEP 04 (2013) 027 [arXiv:1207.5030] [INSPIRE].

[55] T. Gehrmann, S. Hoche, F. Krauss, M. Schönherr and F. Siegert, NLO QCD matrix elements + parton showers in $e^{+} e^{-} \rightarrow$ hadrons, JHEP 01 (2013) 144 [arXiv:1207.5031] [INSPIRE].

[56] L. Lönnblad and S. Prestel, Merging multi-leg NLO matrix elements with parton showers, JHEP 03 (2013) 166 [arXiv:1211.7278] [INSPIRE].

[57] R. Frederix and S. Frixione, Merging meets matching in MC@NLO, JHEP 12 (2012) 061 [arXiv: 1209.6215] [INSPIRE].

[58] R. Frederix et al., A study of multi-jet production in association with an electroweak vector boson, arXiv: 1511.0084 .

[59] A. Denner, S. Dittmaier and L. Hofer, COLLIER - A fortran-library for one-loop integrals, PoS (LL2014) 071 [arXiv: 1407.0087] [INSPIRE].

[60] A. Denner and S. Dittmaier, Reduction of one loop tensor five point integrals, Nucl. Phys. B 658 (2003) 175 [hep-ph/0212259] [INSPIRE].

[61] A. Denner and S. Dittmaier, Reduction schemes for one-loop tensor integrals, Nucl. Phys. B 734 (2006) 62 [hep-ph/0509141] [INSPIRE].

[62] A. Denner and S. Dittmaier, Scalar one-loop 4-point integrals, Nucl. Phys. B 844 (2011) 199 [arXiv: 1005.2076] [INSPIRE].

[63] G. Ossola, C.G. Papadopoulos and R. Pittau, CutTools: a program implementing the OPP reduction method to compute one-loop amplitudes, JHEP 03 (2008) 042 [arXiv:0711.3596] [INSPIRE].

[64] G. Ossola, C.G. Papadopoulos and R. Pittau, Reducing full one-loop amplitudes to scalar integrals at the integrand level, Nucl. Phys. B 763 (2007) 147 [hep-ph/0609007] [INSPIRE].

[65] A. van Hameren, OneLOop: for the evaluation of one-loop scalar functions, Comput. Phys. Commun. 182 (2011) 2427 [arXiv:1007.4716] [InSPIRE].

[66] A. Denner, S. Dittmaier, S. Kallweit and S. Pozzorini, NLO QCD corrections to $W W b b$ production at hadron colliders, Phys. Rev. Lett. 106 (2011) 052001 [arXiv:1012.3975] [INSPIRE].

[67] A. Denner, S. Dittmaier, S. Kallweit and S. Pozzorini, NLO QCD corrections to off-shell top-antitop production with leptonic decays at hadron colliders, JHEP 10 (2012) 110 [arXiv:1207.5018] [INSPIRE].

[68] A. Denner, L. Hosekova and S. Kallweit, $N L O Q C D$ corrections to $W^{+} W^{+} j j$ production in vector-boson fusion at the LHC, Phys. Rev. D 86 (2012) 114014 [arXiv:1209.2389] [INSPIRE]. 
[69] F. Cascioli, S. Kallweit, P. Maierhöfer and S. Pozzorini, A unified NLO description of top-pair and associated Wt production, Eur. Phys. J. C 74 (2014) 2783 [arXiv:1312.0546] [INSPIRE].

[70] M. Grazzini, S. Kallweit, D. Rathlev and A. Torre, $Z \gamma$ production at hadron colliders in NNLO QCD, Phys. Lett. B 731 (2014) 204 [arXiv:1309.7000] [INSPIRE].

[71] F. Cascioli et al., ZZ production at hadron colliders in NNLO QCD, Phys. Lett. B 735 (2014) 311 [arXiv: 1405.2219] [INSPIRE].

[72] T. Gehrmann et al., $W^{+} W^{-}$production at hadron colliders in next to next to leading order QCD, Phys. Rev. Lett. 113 (2014) 212001 [arXiv: 1408.5243] [InSPIRE].

[73] M. Grazzini, S. Kallweit and D. Rathlev, $W \gamma$ and $Z \gamma$ production at the LHC in NNLO QCD, JHEP 07 (2015) 085 [arXiv: 1504.0133].

[74] M. Grazzini, S. Kallweit, D. Rathlev and M. Wiesemann, Transverse-momentum resummation for vector-boson pair production at NNLL + NNLO, JHEP 08 (2015) 154 [arXiv: 1507.0256].

[75] M. Grazzini, S. Kallweit and D. Rathlev, ZZ production at the LHC: fiducial cross sections and distributions in NNLO QCD, Phys. Lett. B 750 (2015) 407 [arXiv:1507.0625].

[76] S. Höche, F. Krauss, M. Schönherr and F. Siegert, $W+n$-jet predictions at the Large Hadron Collider at next-to-leading order matched with a parton shower, Phys. Rev. Lett. 110 (2013) 052001 [arXiv: 1201.5882] [INSPIRE].

[77] F. Krauss, R. Kuhn and G. Soff, $A M E G I C++$ 1.0: a matrix element generator in $\mathrm{C}++$, JHEP 02 (2002) 044 [hep-ph/0109036] [INSPIRE].

[78] T. Gleisberg and S. Hoeche, Comix, a new matrix element generator, JHEP 12 (2008) 039 [arXiv:0808.3674] [INSPIRE].

[79] S. Catani and M.H. Seymour, A general algorithm for calculating jet cross-sections in NLO QCD, Nucl. Phys. B 485 (1997) 291 [Erratum ibid. B 510 (1998) 503] [hep-ph/9605323] [INSPIRE].

[80] S. Catani, S. Dittmaier, M.H. Seymour and Z. Trócsányi, The dipole formalism for next-to-leading order QCD calculations with massive partons, Nucl. Phys. B 627 (2002) 189 [hep-ph/0201036] [INSPIRE].

[81] M. Cacciari, G.P. Salam and G. Soyez, The anti- $k_{t}$ jet clustering algorithm, JHEP 04 (2008) 063 [arXiv: 0802.1189] [InSPIRE].

[82] E. N. Glover and A. Morgan, Measuring the photon fragmentation function at LEP, Z. Phys. C 62 (1994) 311.

[83] A. Gehrmann-De Ridder, T. Gehrmann and E.W.N. Glover, Radiative corrections to the photon +1 jet rate at LEP, Phys. Lett. B 414 (1997) 354 [hep-ph/9705305] [INSPIRE].

[84] A. Gehrmann-De Ridder and E.W.N. Glover, Final state photon production at LEP, Eur. Phys. J. C 7 (1999) 29 [hep-ph/9806316] [INSPIRE].

[85] ATLAS collaboration, A measurement of the ratio of the production cross sections for $W$ and $Z$ bosons in association with jets with the ATLAS detector, Eur. Phys. J. C 74 (2014) 3168 [arXiv: 1408.6510] [INSPIRE].

[86] Particle Data Group collaboration, K.A. Olive et al., Review of particle physics, Chin. Phys. C 38 (2014) 090001 [INSPIRE].

[87] LHC Higgs Cross Section Working Group collaboration, J.R. Andersen et al., Handbook of LHC Higgs cross sections: 3. Higgs properties, arXiv:1307.1347 [INSPIRE]. 
[88] NNPDF collaboration, R.D. Ball et al., Parton distributions with QED corrections, Nucl. Phys. B 877 (2013) 290 [arXiv: 1308.0598] [InSPIRE].

[89] A. Buckley et al., LHAPDF6: parton density access in the LHC precision era, Eur. Phys. J. C 75 (2015) 132 [arXiv:1412.7420] [INSPIRE].

[90] C.M. Carloni Calame, G. Montagna, O. Nicrosini and A. Vicini, Precision electroweak calculation of the production of a high transverse-momentum lepton pair at hadron colliders, JHEP 10 (2007) 109 [arXiv:0710.1722] [INSPIRE].

[91] S. Dittmaier and M. Huber, Radiative corrections to the neutral-current Drell-Yan process in the Standard Model and its minimal supersymmetric extension, JHEP 01 (2010) 060 [arXiv:0911.2329] [INSPIRE].

[92] S. Hoeche, F. Krauss, S. Schumann and F. Siegert, QCD matrix elements and truncated showers, JHEP 05 (2009) 053 [arXiv:0903.1219] [INSPIRE].

[93] SM MC Working Group, SM and NLO MULTILEG Working Group collaboration, J. Alcaraz Maestre et al., The SM and NLO multileg and SM MC working groups: summary report, arXiv: 1203.6803 [INSPIRE].

[94] S. Dittmaier, A general approach to photon radiation off fermions, Nucl. Phys. B 565 (2000) 69 [hep-ph/9904440] [INSPIRE].

[95] S. Dittmaier, A. Kabelschacht and T. Kasprzik, Polarized QED splittings of massive fermions and dipole subtraction for non-collinear-safe observables, Nucl. Phys. B 800 (2008) 146 [arXiv: 0802.1405] [inSPIRE].

[96] S. Hoche, F. Krauss, M. Schönherr and F. Siegert, NLO matrix elements and truncated showers, JHEP 08 (2011) 123 [arXiv: 1009.1127] [INSPIRE].

[97] S. Höche et al., Triple vector boson production through Higgs-Strahlung with NLO multijet merging, Phys. Rev. D 89 (2014) 093015 [arXiv: 1403.7516] [INSPIRE].

[98] S. Catani, F. Krauss, R. Kuhn and B.R. Webber, QCD matrix elements + parton showers, JHEP 11 (2001) 063 [hep-ph/0109231] [INSPIRE].

[99] F. Krauss, Matrix elements and parton showers in hadronic interactions, JHEP 08 (2002) 015 [hep-ph/0205283] [INSPIRE].

[100] S. Frixione and B.R. Webber, Matching NLO QCD computations and parton shower simulations, JHEP 06 (2002) 029 [hep-ph/0204244] [INSPIRE].

[101] S. Frixione, P. Nason and B.R. Webber, Matching NLO QCD and parton showers in heavy flavor production, JHEP 08 (2003) 007 [hep-ph/0305252] [INSPIRE].

[102] S. Hoeche, F. Krauss, M. Schönherr and F. Siegert, A critical appraisal of NLO+PS matching methods, JHEP 09 (2012) 049 [arXiv:1111.1220] [INSPIRE].

[103] S. Höche and M. Schöherr, Uncertainties in next-to-leading order plus parton shower matched simulations of inclusive jet and dijet production, Phys. Rev. D 86 (2012) 094042 [arXiv:1208.2815] [INSPIRE].

[104] S. Schumann and F. Krauss, A parton shower algorithm based on Catani-Seymour dipole factorisation, JHEP 03 (2008) 038 [arXiv: 0709.1027] [inSPIRE].

[105] S. Hoeche, S. Schumann and F. Siegert, Hard photon production and matrix-element parton-shower merging, Phys. Rev. D 81 (2010) 034026 [arXiv:0912.3501] [INSPIRE].

[106] M. Schönherr and F. Krauss, Soft photon radiation in particle decays in SHERPA, JHEP 12 (2008) 018 [arXiv: 0810.5071] [INSPIRE]. 
[107] F. Cascioli et al., Precise Higgs-background predictions: merging NLO QCD and squared quark-loop corrections to four-lepton + 0,1 jet production, JHEP 01 (2014) 046 [arXiv: 1309.0500] [INSPIRE].

[108] S. Hoeche, J. Huang, G. Luisoni, M. Schönherr and J. Winter, Zero and one jet combined next-to-leading order analysis of the top quark forward-backward asymmetry, Phys. Rev. D 88 (2013) 014040 [arXiv: 1306.2703] [INSPIRE].

[109] S. Hoeche, F. Krauss and M. Schönherr, Uncertainties in MEPS@NLO calculations of h+jets, Phys. Rev. D 90 (2014) 014012 [arXiv:1401.7971] [INSPIRE].

[110] S. Hoeche et al., Next-to-leading order QCD predictions for top-quark pair production with up to two jets merged with a parton shower, Phys. Lett. B 748 (2015) 74 [arXiv: 1402.6293] [INSPIRE].

[111] M. Buschmann et al., Mass effects in the Higgs-gluon coupling: boosted vs. off-shell production, JHEP 02 (2015) 038 [arXiv:1410.5806] [INSPIRE].

[112] D. Goncalves, F. Krauss, S. Kuttimalai and P. Maierhöfer, Higgs-strahlung: merging the NLO Drell-Yan and loop-induced $0+1$ jet multiplicities, Phys. Rev. D 92 (2015) 073006 [arXiv: 1509.0159].

[113] A. Buckley et al., Rivet user manual, Comput. Phys. Commun. 184 (2013) 2803 [arXiv: 1003.0694] [INSPIRE].

[114] S. Catani, B.R. Webber and G. Marchesini, QCD coherent branching and semiinclusive processes at large $x$, Nucl. Phys. B 349 (1991) 635 [InSPIRE]. 